National Bureau of Standards

U.S. Department of Commerce

Boulder, Colorado 80303

September 1984 



\section{MICROWAVE DETECTION OF LOST \\ WELLS AND UNKNOWN WATER-FILLED VOIDS IN COAL MINES}

D. R. Belsher

R. H. McLaughlin

A. G. Repjar

H. E. Bussey

Electromagnetic Fields Division National Engineering Laboratory National Bureau of Standards U.S. Department of Commerce Boulder, Colorado 80303

September 1984

Prepared for

Bureau of Mines

United States Department of the Interior

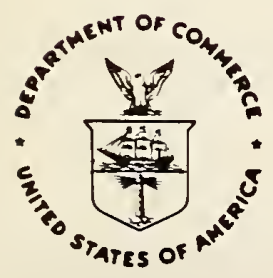

U.S. DEPARTMENT OF COMMERCE, Malcolm Baldrige, Secretary 


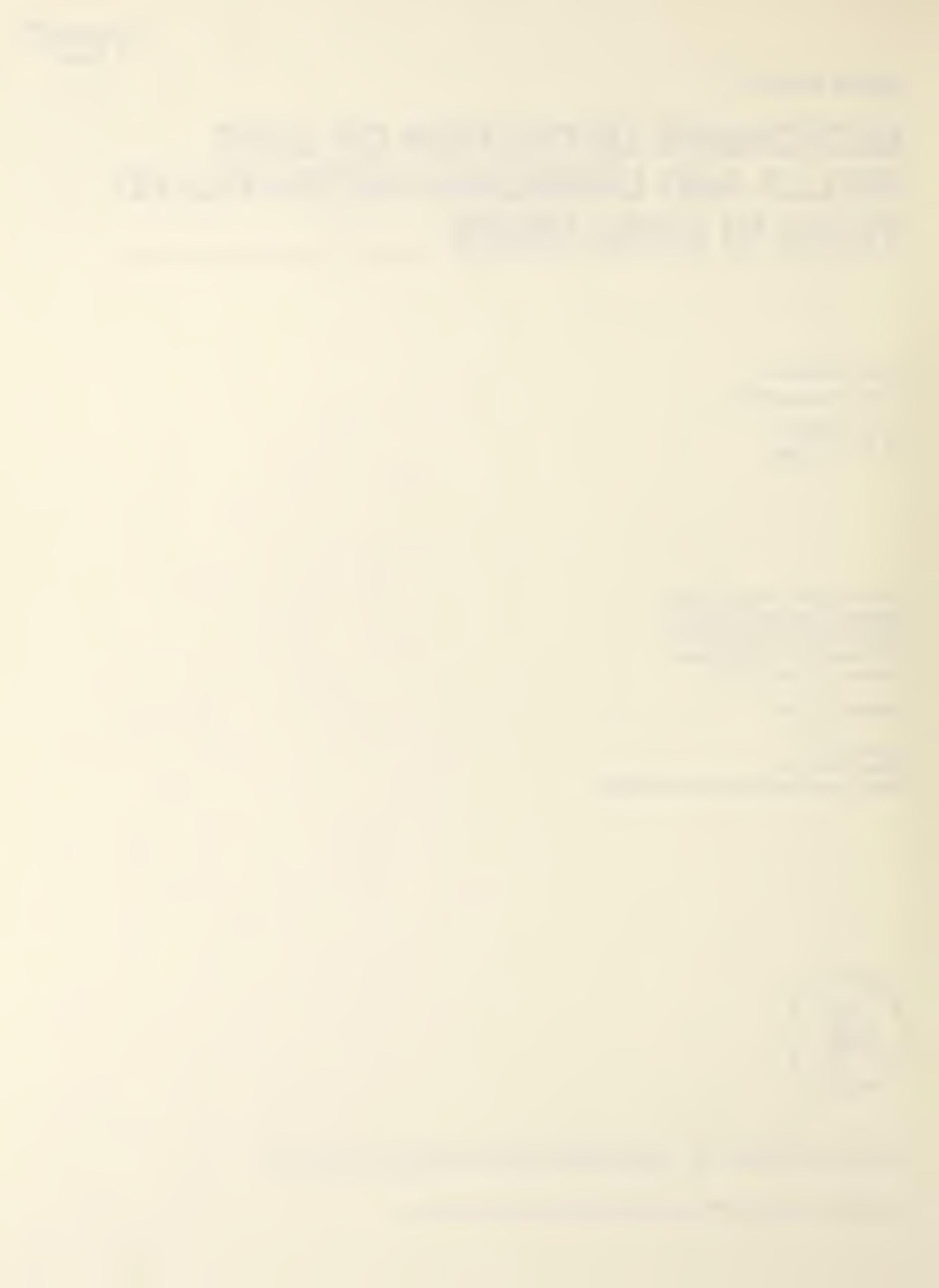




\begin{tabular}{|c|c|c|}
\hline $\begin{array}{l}\text { REPORT DOCUMENTATION } \\
\text { PAGE }\end{array}$ & 1. REPONT NO. & I Reciprents nocenten Ma \\
\hline \multirow{2}{*}{\multicolumn{2}{|c|}{$\begin{array}{l}\text { Microwave Detection of Lost Wells and Unknown } \\
\text { Water-Filled Voids in Coal Mines }\end{array}$}} & S Ropor Dember 1984 \\
\hline & & a \\
\hline $\begin{array}{l}\text { 7. Aurtion(s) } \\
\text { D.R. Belsher, R. }\end{array}$ & H. McLauahlin, A,G. Repiar, H.E. Bussey & $\begin{array}{l}\text { 8. Purromina Ontantiation Avot Na } \\
\text { NBSIR } 34-3017\end{array}$ \\
\hline \multirow{2}{*}{\multicolumn{2}{|c|}{$\begin{array}{l}\text { Electromagnetic Fields Division } \\
\text { National Bureau of Standards } \\
\text { U.S. Department of Commerce } \\
\text { Boulder, Colorado } 80303\end{array}$}} & $\begin{array}{l}\text { 10. Prowees rinezk/Wort Unte Ma. } \\
7233410\end{array}$ \\
\hline & & 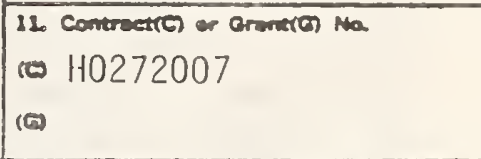 \\
\hline \multirow{2}{*}{\multicolumn{2}{|c|}{$\begin{array}{l}\text { United States Department of the Interior } \\
\text { U.S. Bureau of Mines, Denver Mining Research Center } \\
\text { Denver Federal Center, Denver, Colorado } 80225\end{array}$}} & $\begin{array}{l}\text { 41. To of } \\
\text { Final - Phase I I } \\
\text { Jan. } 1979 \text { to March } 1984\end{array}$ \\
\hline & & 14 \\
\hline
\end{tabular}

14. supdementary mete

14. Abetrea (Limit 200 wonts)

Work on contract $H 0272007$ is summarized for the period of January 1979 through March 1984. The development of improved antennas useable with both a pulse system or an FM-CW system is described. The development of a field prototype pulse sampling system is described. Initial theoretical work on the problem of dielectric loading of antennas as well as a study of notential system range is included.

17. Doeument Anatreve a Docoriptore

Detection of mining hazards

Detection of coal mining hazards

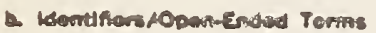

c. cosati nold/croup

12. Avallabisicy statement

Available from NTIS

\begin{tabular}{|c|c|}
\hline 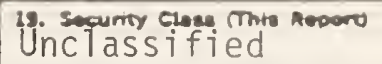 & 21. whe of now \\
\hline Unclassified & 2 \\
\hline
\end{tabular}




\section{FOREWORD}

This report was prepared by the National Bureau of Standards (NBS), Electromagnetic Fields Division, Boulder, Colorado, under USBM Contract number H0272007. The contract was initiated under the Minerals Health and Safety Program. It was administered under the technical direction of the Premining Hazards Division, Denver Research Center, with Mr. Richard L. Myers acting as Technical Project Officer. Mr. Dennis Maez was the contract administrator for the Bureau of Mines. This report is a summary of the work recently completed as a part of this contract during the period 23 January 1979 to 1 March 1984. This report was submitted by the authors on 1 May 1984 and published in September 1984 .

The NBS thanks other staff members not named as authors who contributed indirectly to this work: Doyle A. Ellerbruch for helpful discussions on techniques, Douglas T. Tamura for assistance in the field, and James R. Andrews for helpful discussions on pulse theory.

The authors also wish to acknowledge the considerable help provided by the staff of the Kaiser Coal Mine at Raton, NM, especially that of Mr. Ed Moore, Mr. Richard E. Hood, Mr. Rod Lawrence, Mr. Terry Jordan, Mr. Ron Chavez, and Mr. Harry H. Elkin.

The assistance provided by personnel of the Bureau of Mines, Denver Research Center, is greatly appreciated, most notably that of project monitor Mr. Richard L. Myers and also Mr. R. J. Leckenby.

The authors regret the unavoidable delay in concluding this project. Progress was stopped for more than two years while manufacturers of critical sub-assemblies worked to meet the specifications. 


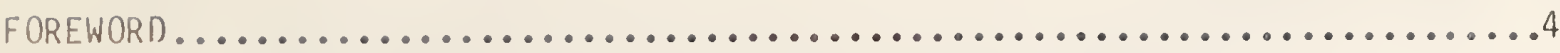

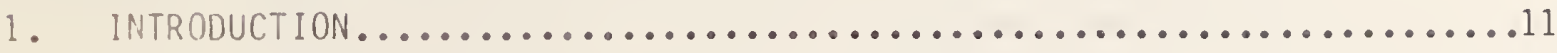

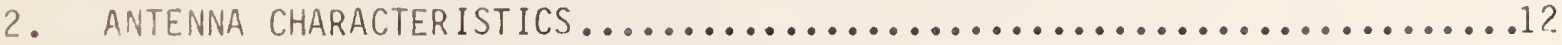

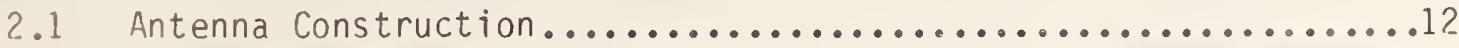

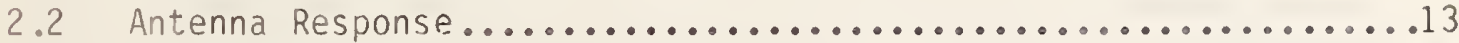

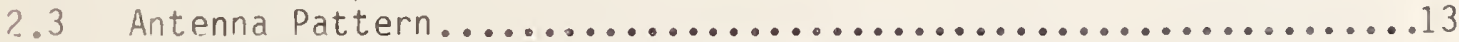

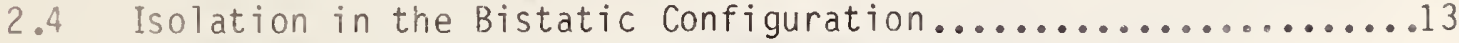

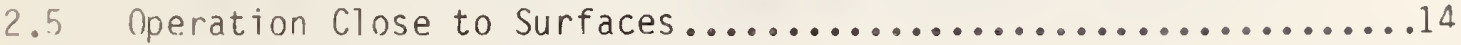

3. POTENTIAL FOR DIELECTRIC LOADING OF ANTENNAS ..................14

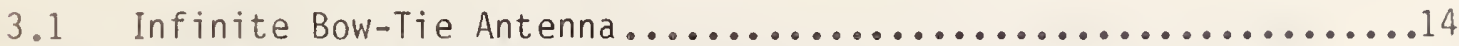

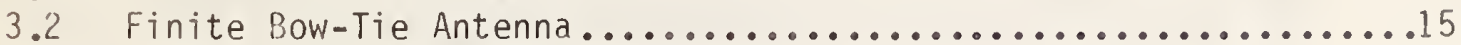

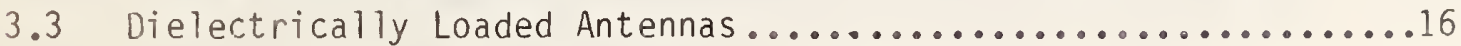

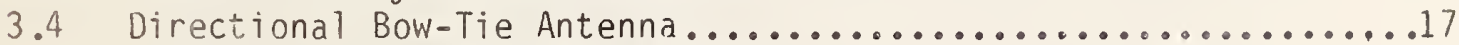

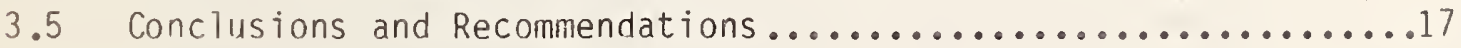

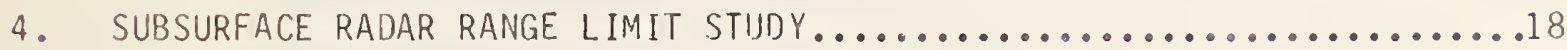

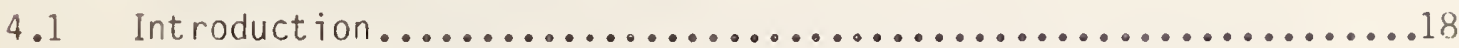

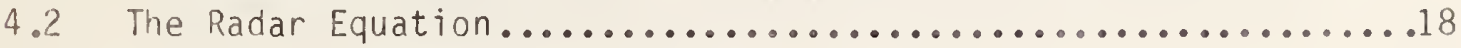

4.3 Interface Transformation Factors, I $c_{d} \ldots \ldots \ldots \ldots \ldots \ldots \ldots \ldots \ldots \ldots . \ldots \ldots \ldots$

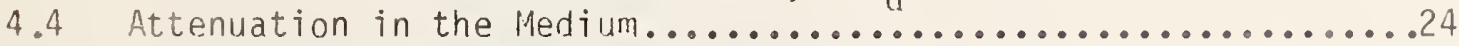

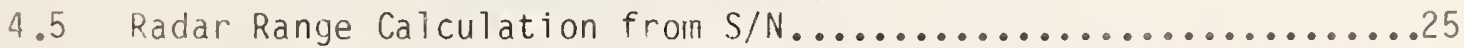

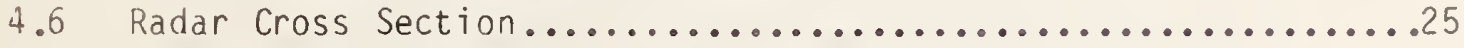

4.7 Electromagnetic Theory of Scattering Cross Sections

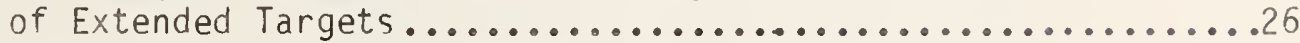

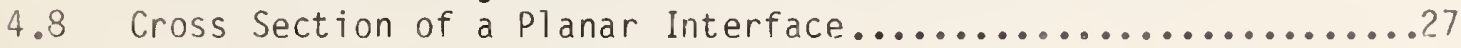

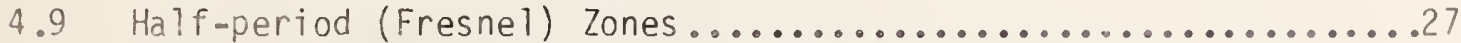

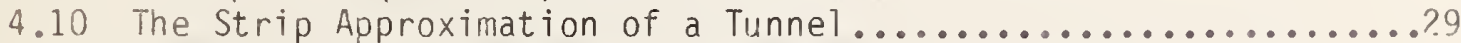

4.11 Cross Section of a Dielectric Tunnel at Broadside Incidence....30

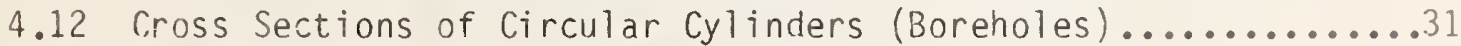

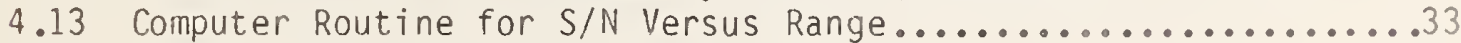

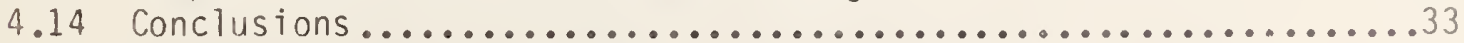

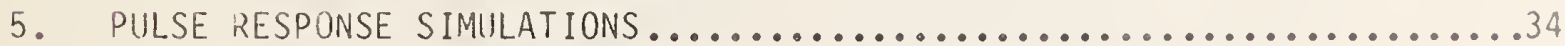

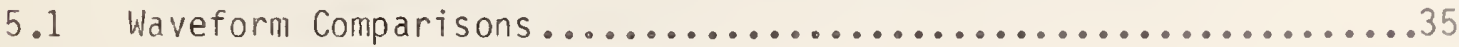

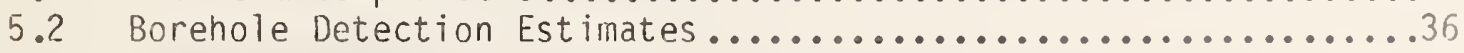

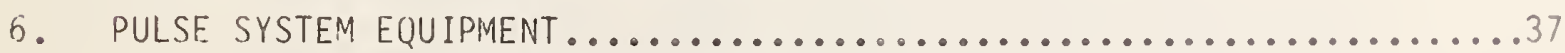

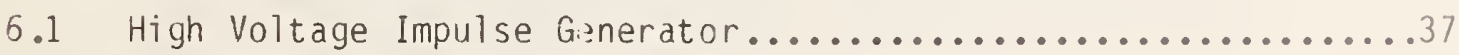

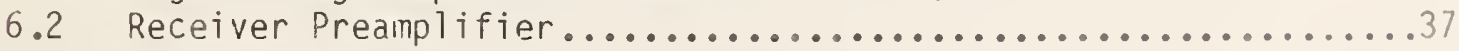

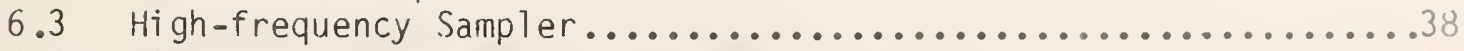

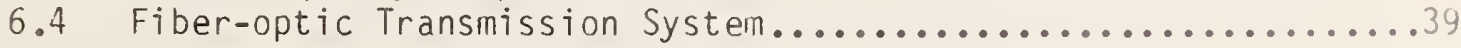

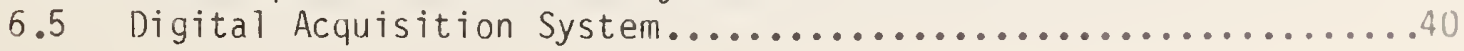




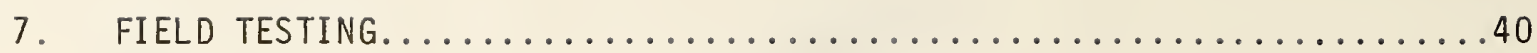

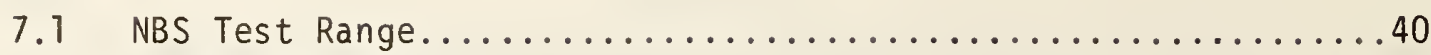

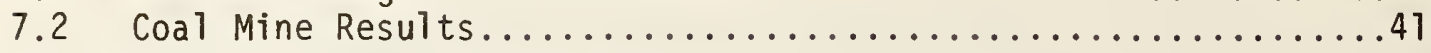

8. ADAPTATION OF SEISMIC DATA METHODS......................... 41

9. FUTURE DIRECTIONS FOR RADAR DETECTION OF UNDERGROUND ANOMALIES .....42

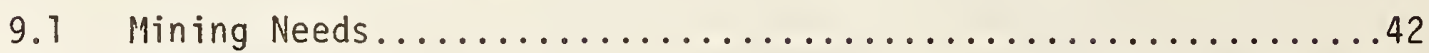

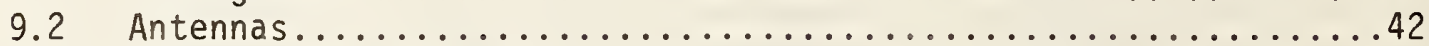

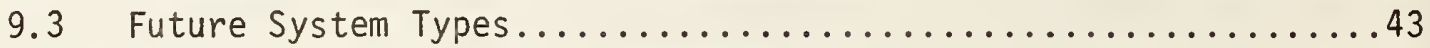

9.4 Accessibility to Mine Test Areas...................... 43

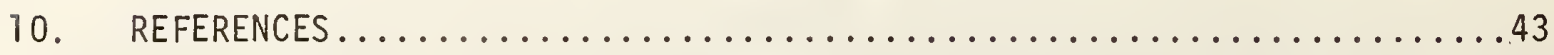

APPENDIX A. COMPUTER PROGRAM FOR SIGNAL-TO-NOISE RATIO, AS A FUNCTION

OF DISTANCE (RANGE) TO A SUBSURFACE TARGET............ 82 
Figure 1. Reflections due to the NBS-TQ antenna without the resistive material (1) and the same antenna with

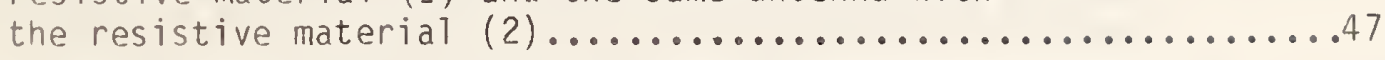

Figure 2. Transmitted waveform from the TG antenna (curve 1) and the

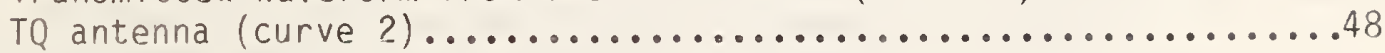

Figure 3. Relative transmission in $\mathrm{dB}$ for the TG antenna (plain line) forward direction, the TQ antenna (squares) forward direction, and the TQ antenna (triangles) reverse direction at a range of $3 / 4 \mathrm{~m}$.

Figure 4. Baffle arrangement for isolating TQ antennas when operated bistaticaliy

Figure 5. Response (1) of the system with the TQ antenna pair pointed at the sky and (2) to a 1.2 meter square metal plate $5 \mathrm{~cm}$ in front of the baffle.

Figure 6. Pulse system antenna return from an air-filled box target buried at 0.6 meter (1) and the same return corrected by subtraction of the sky return (2)

Figure 7. Non-coplanar fin antenna schematic...

Figure 8. Characteristic impedance of the non-coplanar fin antenna with equal fin angles (plotted from data presented by

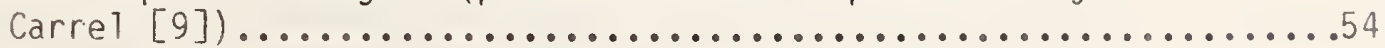

Figure 9. Input impedance of biconical antennas with $5.4^{\circ}$ cone angle and $0.054^{\circ}$ cone angle as a function of the length, $\ell$, of the cone in wavelengtis, $\lambda$ ( $p$ lotted from data presented by

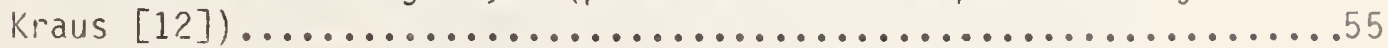

Figure 10. Input impedance of single cones with ground plane as a function of cone length, $l$, in wavelengths, $\lambda$. Cone angles are $10^{\circ}, 20^{\circ}, 40^{\circ}$, and $60^{\circ}$ (plotted from data presented by Kraus [12]).

Figure 11. Input impedance of single cone with ground plane versus $k \ell$. $K$ is the wave number, $\ell$ is the cone length, and the cone angle, $\theta_{0}$, is $60^{\circ}$ ( $p$ lotted from data presented by Papas and King [13]).

Figure 12. Characteristic impedance of a biconical antenna with equal cone angles, $\Psi_{0}$ (plotted from data presented by Carrel [y]) .....5. 
Figure 13a. Sketch of the rays from source to field point (target). Rays are refracted into the second medium and partially reflected, $n_{0}<n_{1}$

Figure 13b. Geometry for calculating Sommerfeld interface transmission. In this example, the source is in denser medium with refractive index $n_{0}>n_{1} \ldots$

Figure 14. Signal-to-noise ratio for a $\mathrm{CW}$ radar system with the following parameters: $500 \mathrm{MHz}$ source; horn antennas $50.8 \mathrm{~cm}$ $x 76.2 \mathrm{~cm}$; frequency $=0.5 \mathrm{GHz}$; complex permittivity $=9-j 0$ for low loss curves, 4-j0.16 for medium loss curves, 9-j0.9 for high loss curves. The upper curve of each group is for a flat mine tunnel wall $1.5 \mathrm{~m}$ high of infinite length with water in the tunnel. The lower solid curve of each group is for a $30 \mathrm{~cm}$ diameter cased borehole. The dashed curve is for a water-filled borehole without casing having a 30 $\mathrm{cm}$ diameter. The system noise is assumed to be $8 \mathrm{~dB}$ greater than Johnson noise in a $1 \mathrm{MHz}$ bandwidth and clutter

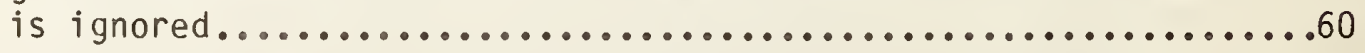

Figure 15. Simulated impulse waveforms having widths of 5, 2, 1, and

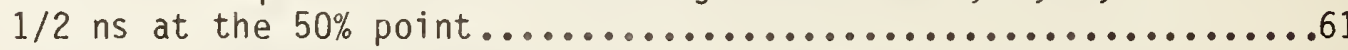

Figure 16. Resulting spectral energy distribution of the impulses shown in figure 15 in $\mathrm{dB}$ relative to $10^{-9}$ volt-seconds........62

Figure 17. The signals resulting from transmission of the impulses shown in figure 15 over a 1.5 meter path with the TQ antennas.

Figure 18. Simulated sinusoidal pulse waveforms having $8,4,2$, and 1 c.ycles. .64

Figure 19. Riesultant spectral amplitude of the sinusoidal pulses shown in figure 18. 8-cycle pulse, dotted curve; 4-cycle pulse, triangles; 2-cycle pulse, squares; 1-cycle pulse, solid

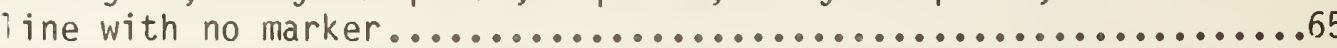

Figure 20. The signals resulting from the transmission of the sinusoidal pulse waveforms shown in figure 18 over a 1.5 meter path using TQ antennas..............................6

Figure 21. Simulated linear chirp pulse waveforms. 250-500 MHz in 10 nanoseconds, plain curve. 250-1000 MHz in 10 nanoseconds, square markers. 250-1000 MHz in 20 nanoseconds, triangle markers.........................67

Figure 22. Resulting spectral amplitudes of the linear chirp pulse waveforms shown in figure 21 . The curve markers correspond t.o figure 21 
Figure 23. Resulting signals after transmission of the linear chirp pulses over a 1.5 meter path using TQ antennas. The curve markers correspond to figure $21 \ldots \ldots \ldots \ldots \ldots \ldots \ldots . . \ldots \ldots 9 . \ldots \ldots$

Figure 24. The result of transmitting a 300-600 MHz linear chirp pulse over a 1.5 meter path using the TQ antennas ................70

Figure 25. The result of autocorrelation of the waveform of figure $24 \ldots \ldots 1$

Figure 26. Autocorrelation of the transmitted waveform of the 4 cycle

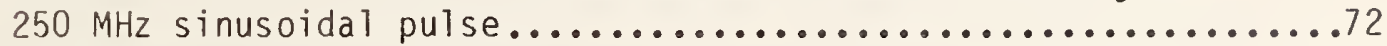

Figure 27. Simulated response in relatively damp coal for a $15 \mathrm{~cm}$ radius hole at 1 meter range. (1) cased hole, (2) air-

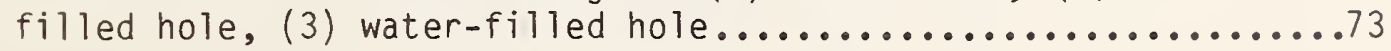

Figure 28. Simulated response in relatively damp coal for a 15-crn radius hole at a range of 1 meter (1), 2 meters (2), and 5 meters (3). The amplitude of the response at 5 meters has

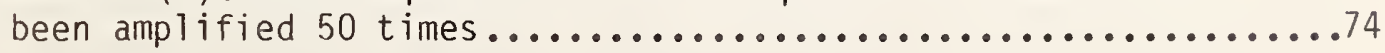

Figure 29. Simulated radar response in relatively damp coal for a 1.0 volt, 1 nanosecond input pulse reflected from a borehole. The given dimension is the borehole radius ...................

Figure 30. Simulated radar response in relatively dry coal for a 1.0 volt, 1 nanosecond input pulse reflected from a borehole. The given dimension is the borehole radius ..................

Figure 31. Preprototype pulse system block diagram...................77

Figure 32. Output waveform of the high-voltage impulse

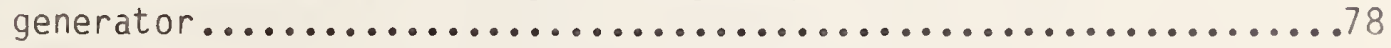

Figure 33 . NSB range for subsurface anomaty measurements ..............79

Figure 34. Pulse system response averaged over a sequence of 26 measurements (1). Single response from over the 0.6 meter deep air-filled target (2). The resulting response showing the target signal enhancement (arrow) obtained by subtracting sequence average from the response at one position (3) ........80

Figure 35. A stacked series of modified responses obtained by subtracting the average response of the series from each measured response. Target signal at arrow..............81 


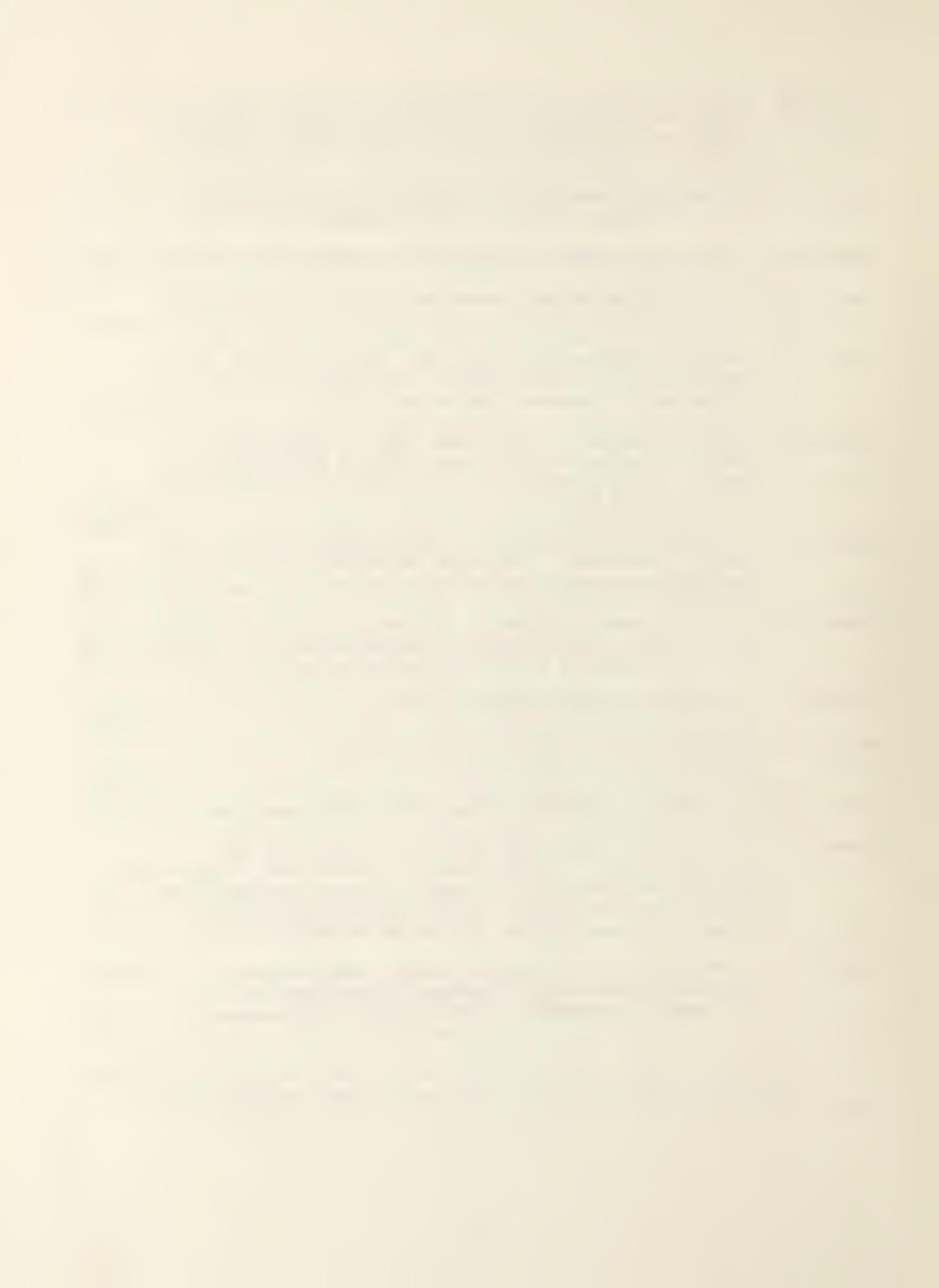




\title{
MICROWAVE DETECTION OF LOST OIL WELLS AND UNKNOWN WATER-FILLED VOIDS IN COAL MINES
}

\author{
D. R. Belsher (retired), R. H. McLaughlin, \\ A. G. Repjar, H. E. Bussy (retired) \\ Interference Characterization Group \\ Electromagnetic Fields Division \\ National Bureaul of Standards \\ Boulder, C0 80303
}

\section{INTRODUCTION}

This is the final report for work (Phase II) carried out by the National Bureau of Standards (NBS) under contract $\mathrm{H} 0272007$ with the Bureau of Mines.

Many thousands of oil wells pass through the coal beds of the United States. Of these, a very large number are in active underground coal mining areas. The casings of these wells are found, when possible, on the surface, and their underground positions are determined from drilling data or costly wel1-logging techniques. Many of these wells were drilled and abandoned years ago and are often impossible to locate at the surface. The drilling logs for those that can be located at the surface often do not exist. These "lost" wells are believed to have caused some major coal mine explosions. Some coal mine explosions have resulted in the loss of over a hundred lives in a single catastrophic event. The hazard is produced when the cutter teeth of a mining inachine contact and penetrate a steel well casing. The resulting sparks can easily ignite the casing gas and the coal gas and dust present at the inining face. Where the location of a well casing is known, the law requires a coal pillar to be left surrounding the underground position. Fven wells whose positions are known both on the surface and underground may be penetrated when new or inexperienced personnel misjudge their underground positions. The underground position of a cased well is often different from the surface position since many wells are not drilled vertically. As a result, mine managers must constantly check for any one of the many thousands of lost or undocumented cased and uncased wells.

Flooding is another coal mine hazard. Experience has shown that flooding generally occurs when coal is mined within approximately $3 \mathrm{~m}$ (10 ft) of a flooded area in a previously worked mine. Severe flooding can also occur by cutting into a water-filled well. This happens even when $6.10 \mathrm{~m}$ (20 foot) horizontal drilling guidelines are followed and mining is restricted to more than $15.3 \mathrm{~m}$ (50 feet) from old workings. Testing for old workings by driling is often a cause of dangerous flooding since the drill hole can easily be enlarged by water flow. Also, old workings can be missed when mining personnel test by drilling. 
A microwave technique appears to offer an excellent chance for success in detecting voids and well casings to ranges of $15 \mathrm{~m}$ (50 feet) or more. All of the prominent microwave techniques have potential advantages over other techniques in that:

(1) The antenna need not physically contact the working face;

(2) the antenna can be mounted on room and pillar mining machinery, or on longwall mining machinery, or it can be hand carried;

(3) ineasurements can be made quickly with minimum interference to production; and

(4) data can be analyzed and made available for immediate use by production personnel.

It was felt that the maximum benefit, for the available tirne and money provided in Phase I of this work, would be achieved by improvements in the antennas which would be useful for any microwave systern. Accordingly, in Phase II of this work, the goals were to provide additional antenna improvements and to assemble a pre-prototype impulse radar system for delivery to the Bureau of Mines. Additional effort has been expended on studies of potential range (see section 4) and a study of dielectric loading of antennas (see section 3). A short comparison study of possible pulse types useable in underground radar has been included (see section 5). The general layout of the prototype impulse system is described.

\section{ANTENNA CHARACTERISTICS}

\subsection{Antenna Construction}

During Phase I of this contract, a broadband, TEM-type antenna was developed. Designated the NBS-TG, it consisted of two tapered leaves and had an impedance which varied exponentially from 50 ohms at the feed to 377 ohms at the aperture. The antenna has been improved by encasing it in a shielded enclosure and is now known as the NBS-TQ. This shielding improves the frontto-back ratio as well as the isolation when the antennas are used bistatically. Shielding was added by placing the antenna in a metal box having an aperture at the open end of $50 \mathrm{~cm}$ by $80 \mathrm{~cm}$. The box was $63 \mathrm{~cm}$ deep and was lined with a 3/4-inch thickness of a commercially available conductive foam to suppress surface currents in the box. Additionally, two sheets of resistive material (approximately 100 ohms per square) $8 \mathrm{~cm}$ wide by $15 \mathrm{~cm} 1 \mathrm{ong}$ were riveted to the tips of the antenna halves. The resistive sheets were not terminated on the box but were spaced away from the metal. The resistive strips were added to provide lower reflections at the aperture. Figure 1 shows a comparison in the time domain of the reflections due to the NBS-TQ antenna without the resistive material, curve (1) and the same antenna with the resistive material added, curve (2). Notice that the right-hand peak, which is the aperture reflection, is much reduced. The incident signals were $1 / 2$ ns duration irnpulses of equal amplitude. 
The antenna feed is by way of a type $\mathrm{N}$ feedthrough connector at the box and a $0.6-m$ length of $R G-58$ coaxial cable connected to the antenna using an SMA connector. The remaining space in the shielding box is filled with low density styrofoam to completely fill the box and support the antenna firmly in position. The aperture was covered with sheet plastic and sealed with heavyduty tape.

\subsection{Antenna Response}

The measured transmitted pulse response, using an electrically small resistive dipole as a standard receiving antenna, of both the NBS-TG and the NBS-TQ antennas is compared in figure 2. It is obvious that the isolating box does introduce some changes in the transmitted waveform, but the reduction in transmission to the sides and back should make the overall system performance much better.

Unless otherwise noted, al1 measurements shown were made with the equipment described in the Phase I Final Report for this contract. That system included a commercially available digital-processing oscilloscope and an NBS-constructed impulse generator having a 45-volt amplitude into 50 ohms and 1/2-ns width (FWHM--full width, half maximum, or 50\% amplitude points).

\subsection{Antenna Pattern}

A comparison of the forward and reverse transmitting transfer function amplitudes with the probe at a $3 / 4 \mathrm{~m}$ distance from the nearest antenna surface of the NBS-TG and the encased version, the NBS-TQ, is shown in figure 3 . The transmitting transfer function characterizes the electromagnetic fields which are generated at a given distance by a known signal applied at the feed and has the units of volts/meter/volt. This concept is discussed in reference [3]. The front-to-back ratio for the NBS-TG is approximately 1; so even with some reduction in forward signal, the improvement in front-to-back ratio for the NBS-TQ is quite worthwhile. The improvement in front-to-back ratio is very important for use in the close confines of a mine and especially where noisy and highly reflective mining machinery is present. Thus, this task was given high priority.

\subsection{Isolation in the Bistatic Configuration}

A pair of the NBS-TQ antennas were mounted with the E-field directions paralle1. They were then tested with different spacings between the antennas, different baffle configurations between the antennas, and different standoff distances from material surfaces. The results of this work generally showed that an optimum spacing between antennas should be about $36 \mathrm{crn}$ with a baffle centered between the antennas and extending about $68 \mathrm{~cm}$ to the front. The most effective baffle tested consisted of a thin dielectric material (1/4-inch plywood) with a sheet of absorber glued to both sides (see figure 4). A metal baffle gave more isolation between antennas, but interacted with the antennas and the ground to give other responses which were undesirable. 


\subsection{Operation Close to Surfaces}

For operation near a coal face, for example, the antennas should change their characteristics as little as possible as the surface is approached. This means that the effect of variations in spacing from the coal surface encountered during use will not be a source of confusion. If the antenna performance is insensitive to distance from the coal face, it is possible, as a first step in processing the measurement data, to subtract a background signal which is common to the system. The background signal can be obtained by pointing the antenna pair at the sky in a position where it is not near reflecting objects. Figure 5 shows a comparison of the response of the TQ antenna pair with the baffle pointed at the sky with the response from a metal plate. Figure 6 shows how this same system sky response may be subtracted from a ground signal to yield an enhanced response from a buried target. It can be noted that even though the target response (see arrow) is down in absolute amplitude, its relative amplitude increase makes it visually more obvious in the corrected waveform.

\section{POTENTIAL FOR DIELECTRIC LOADING OF ANTEMNAS}

The infinite "bow-tie" antenna belongs to the frequency independent class of antennas because its shape is defined entirely by angles $[1,2]$. As a result, its impedance, polarization, pattern, etc., are independent of frequency and the anterna is said to be broadband. The characteristics of such broadband antennas are necessary for accurate impulsive electromagnetic field measurements [3] which can be used for the detection and analysis of prominent features in a geophysical environment [4].

The overall purpose of this section is to investigate the characteristics of a practical, i.e., finite, bow-tie antenna with the following specifications. The antenna should be broadband, matched to a 50-ohm source 7ine, and have some directionality $[5,6]$. The frequency range of interest for this study is specified to be from $10 \mathrm{MHz}$ to $1 \mathrm{GHz}$. To achieve these goals, considerations are given to dielectrically filling the antenna which also increases its electrical size [5]. Resistively loading the antenna to achieve broadbanding from $500 \mathrm{MHz}$ to $1 \mathrm{GHz}$ is currently being studied at NBS $[7,8]$.

First, the characteristics of the infinite bow-tie antenna as a function of its shape $[2,9]$ will be presented. Also, the effect of a dielectric medium on its electrical properties is shown. Next, the "finite" bow-tie antenna is considered, followed by a discussion on dielectrically loading antennas. By the use of non-symmetrical dielectric loading, a directional bow-tie antenna is then investigated. Finally, conclusions and recominendations are presented.

\subsection{Infinite Bow-Tie Antenna}

The problem of finding the characteristic impedance, $z_{0}$, of an antenna consisting of two infinitely long conical conductors which have a common apex has been solved by Carrel [9]. The examples presented include the coaxial biconical antenna [10], the non-coaxial biconical antenna [11], the coplanar fin antenna, and the non-coplanar fin antenna. The infinite bow-tie antenna 
can be simply treated as a special case of either of the latter two examples. See figure 7. Due to recent interest in TEM horns [7], it is appropriate to consider the infinite bow-tie antenna as a special case of the noncoplanar fin antenna (see figure 7). The solution for the characteristic impedance, $Z_{0}$, is given by

$$
z_{0}=n K / K^{\prime}
$$

where $n=\checkmark \frac{\bar{\mu}}{\varepsilon}$, the intrinsic impedance of the medium between the conductors. Here, it is only necessary to mention that $K$ and $K^{\prime}$ are the complete elliptic functions and inevitably depend only on the angles $\psi_{0}$ and $\theta_{0}$ defined in the figure. $\mu$ and $\varepsilon$ are, of course, the permeability and dielectric constant of the medium, respectively.

In figure 8, the characteristic impedance of the non-coplanar fin antenna with equal fin angles is given as a function of $\psi_{0}$ and $\theta_{0}$. For the infinite bow-tie antenna, $\theta_{0}=180$ degrees. It should be noted that the values obtained from figure 8 are for an antenna in free space which has a dielectric constant, $\varepsilon_{0}$.

In a dielectric medium with a relative dielectric constant, $\varepsilon_{r}$, the characteristic impedance, $Z_{0}\left(\varepsilon_{r}\right)$, is

$$
z_{0}\left(\varepsilon_{r}\right)=\frac{z_{0}\left(\varepsilon_{0}\right)}{\sqrt{\varepsilon_{r}}}
$$

where $z_{0}\left(\varepsilon_{0}\right)$ is the characteristic impedance in free space.

It is apparent that the characteristic impedance of an infinite bow-tie antenna thus depends on both the fin angle, $\psi_{0}$, and the dielectric constant, $\varepsilon_{r}$. These factors should be considered when matching the antenna to a 50-ohm source line and/or a dielectric medium.

\subsection{Finite Bow-Tie Antenna}

The input impedance equals the characteristic impedance for the infinite bow-tie antenna and for the infinite biconical antenna [12]. However, the theoretical input impedance has only been obtained for the "finite" biconical antenna. For the finite biconical antenna whose half-angle, $\psi_{0}$, is less than about 3 degrees, the input impedance has been calculated [12]. For the wideangle biconical antenna, the input impedance has been calculated [12, 13$]$. For these cases, it is significant that the wider biconical antenna has not only a lower characteristic impedance, but also an input impedance that is more constant as a function of cone length than the narrower antenna (see figure 9). From this result, it has generally been concluded that wider antennas are more suitable for broadband application. To further substantiate this trend, Kraus shows that, for cones with half angles of $5^{\circ}, 10^{\circ}, 20^{\circ}$, and $30^{\circ}$ 
[12] and characteristic impedances of 188, 146, 104, and 80 ohins, respectively, the input impedance variation reduces with increasing cone angles (see figure 10). It also appears evident that the input impedance varies about the characteristic impedance, as is further seen in figure 11 . The existence of these factors should be determined by measurement for the finite bow-tie antenna so that rnatching to a 50-ohm source line may be accomplished. It should be noted that the results in [12] and [13] are for the cases where the lower cone is replaced by a ground plane. Hence, the impedance values are half of those which would be obtained for the biconical antenna. For reference, the characteristic impedance of the infinite biconical antenna with equal cone angles is given in figure 12 .

\subsection{Dielectrically Loaded Antennas}

The basis for determining the characteristics of the bow-tie antenna in the presence of a dielectric has, in part, been discussed earlier in this section. To extend this basis, it is appropriate to study the cases where either a dipole [14] or biconical antenna [15] is imbedded in a spherical dielectric of radius a, since the case for the bow-tie antenna has not been solved. By letting a approach infinity, one could then study the effect of matching the dielectric filling to the dielectric medium [16].

For the above cases, consider the dielectric sphere to have a dielectric constant, $\varepsilon_{1}$, and let the dielectric constant of the medium be $\varepsilon_{2}$. For the dipole case and for $k_{1} a$ and $k_{2} a \ll 1$, the radiation resistance decreases as $\varepsilon_{1}$ increases [14]. However, the radiation power factor, which denotes the available product of bandwidth and efficiency $[14,17]$, increases only slightly for $1<\varepsilon<4$, where $\varepsilon \equiv \varepsilon_{1} / \varepsilon_{2}$, and decreases for larger values of $\varepsilon$. Over the range $1<\varepsilon<4$ the radiation resistance decreases by a factor of 4 . Hence, although the resistance can be varied to possibly match to a 50-ohm line, which is in itself desired, one might not expect a significant increase in bandwidth.

For the wide-angle biconical antenna and for $k_{1} a$ and $k_{2} a<1$, the radiation power factor decreases as the dielectric loading, $\varepsilon$, increases [15]. Also the conductance is independent of $\varepsilon$. It should be pointed out that the above results assume the electric field to be tangential to the dielectric boundary. However, these results, along with those for the dipole case, may be indicative of what to expect for the bow-tie antenna; that is, that the power factor may not significantly increase and the radiation resistance may decrease as $\varepsilon$ decreases. Dielectric loading may not offer a significant improvernent in antenna performance unless the antenna can be placed in contact with the medium of interest--the coal seam in this case. 
Lytle [6] has shown that a miniaturized directional antenna can be obtained by placing sources offset in material with a high dielectric constant. In a similar manner, one could offset the bow-tie antenna in a dielectric sheet such that the radiation would be maximized in one-half plane and would be minimized in the other half plane. In principal, the wavelength in the dielectric material, $\lambda_{1}$, is

$$
\lambda_{1}=\frac{\lambda_{2}}{\sqrt{\varepsilon}}
$$

where $\lambda_{2}$ is the wavelength in the medium and $\varepsilon=\varepsilon_{1} / \varepsilon_{2}$, where $\varepsilon_{1}$ and $\varepsilon_{2}$ are the dielectric constants in the material and medium, respectively. Hence, by offsetting the antenna froin one side of the dielectric sheet a distance $d_{1}$, such that $d_{1}=(2 \ell+1) \lambda_{1} / 4$, $\&$ an integer, and requiring the width of the inaterial to be such that the antenna is a distance, $d_{2}$, from the other side, where $d_{2}=n \lambda_{1} / 2, n$ an integer, one would maximize the radiation on the former side and minimize the radiation on the latter side. This design can only be optimized over a narrow bandwidth using frequency independent dielectrics. Further work is required in order to develop dielectrics or ferroelectrics which have dielectric constants inversely proportional to the square of frequency over the bandwidth of interest.

\subsection{Conclusions and Recommendations}

The input impedance of an infinite biconical or infinite non-coplanar fin antenna, which may be an infinite bow-tie as a limiting case or an infinite TEM horn, depends only on the angles defining the antenna and on the permeability and dielectric constant of the medium. When these antennas become finite, it appears that the input impedances of the wide-angle antennas are more constant as a function of frequency than are narrow-angle antennas and vary about the characteristic impedance. Such antennas, which include the wide-angle bow-ties, are considered to be broadband.

By enclosing the bow-tie antenna in a sheet of dielectric, one may increase the directionality of the antenna in a given band of frequencies, but the radiation resistance may decrease as the dielectric constant increases.

Based on the above, one can design either a wide-angle TEM horn or bowtie antenna such that the input impedance is known within bounds for a given dielectric medium. Thus, one can optinize the antenna so that its input impedance can be matched to a 50-ohm source to maximize power transfer. Using dielectric media such as air and water, it is recommended that the theory be verified to determine its applicability. In particular, the input impedance for the antenna in both inedia should be measured over the frequency range from $10 \mathrm{MHz}$ to $1 \mathrm{GHz}$. It would then be feasible to increase the directionality of 
the antenna over a frequency range by enclosing the antenna in a dielectric sheet. The accuracy of the theory could be verified and the bandwidth of the antenna immersed in the dielectric sheet could be compared to those obtained for the infinite media.

The above recommendations could effectively be accomplished by using image theory and performing the experiments using a ground plane, thereby avoiding the use of a broadband balun, which can be a drawback for precise and accurate measurements.

\section{SUBSURFACE RADAR RANGE LIMIT STUDY}

\subsection{Introduction}

This section gives theoretical estimates of the radar range limit of buried targets. Cook, in 1974 and 1975 [18,19], investigated the subject. The main parameters of the radar range equation are discussed, and the detection range is found with appropriate receiver noise assumed and no clutter.

The theory needs to be augmented by practical experience with "radar clutter," i.e., reflections from miscellaneous targets. This clutter is rather analogous to precipitation and atmospheric turbulence clutter during aircraft detection. The coal "atmosphere" has inhomogeneities that may act as false targets, giving clutter.

The desired targets considered are a borehole and a mine tunnel. The radar cross sections of these targets are obtained by suitable modifications of known theory for homogeneous dielectric and metaliic cylinders and for a strip approximating an old workings tunnel. An outline of this section is as follows: The radar signal equation is given which contains the source power, the antenna gain and area, the dielectric loss, interface loss, and inverse distance loss of propagation in the medium, and the radar cross section of the target. Cross sections for the targets of interest are derived in some detail, including new results for cross sections that depend on range. Finally, with assumed system parameters, including the noise figure, the radar range equation is solved (Appendix $A$ ).

\subsection{The Radar Equation}

The radar equation is developed in many radar books, e.g., Berkowitz [20]. In our application, the radar back scattered received signal power in a homogeneous lossy atmosphere (the coal) is calculated as

$$
S=\frac{P G_{1}}{4 \pi R^{2}}-\frac{\sigma}{4 \pi R^{2}}-\frac{\lambda_{0}^{2}}{4 \pi} I G_{d} D e^{-4 \alpha R} 1 \text {, }
$$


$P=$ emitted power during the $r f$ pulse, or the effective power level of the "continuous" wave of an FM-CW radar, watts,

$G_{1}, G_{2}=$ gains of transmitting and receiving antennas (gains may differ from specifications if target is closer than diam²/wavelength),

$\sigma=$ target radar cross section area,

$\lambda_{0}=$ wavelength of EM wave in air,

$\alpha=$ attenuation coefficient of the EM field due to the material dielectric, loss specifically, $\alpha=$ the real part of $\left(-\omega^{2} \mu \varepsilon\right)^{1 / 2}$ where $\omega$ is the radian frequency and $\mu$ and $\varepsilon$ are the magnetic permeability and electric permittivity of the material,

$\varepsilon=\varepsilon_{0}\left(\varepsilon^{\prime}-j \varepsilon^{\prime \prime}\right)$, where $\varepsilon_{0}$ is the permittivity of vacuum, and $\varepsilon^{\prime}, \varepsilon^{\prime \prime}$ are the real and imaginary parts of the relative complex permittivity $\varepsilon^{\star}=\varepsilon^{\prime}-j \varepsilon^{\prime \prime}$ (with $\exp (+j \omega t)$ time dependence), and $n \cong \sqrt{\varepsilon}$, a useful refractive index for finding $\theta$ in Snell's law.

$I=$ the reflection power loss at the air-to-material interface counting both the entrance and the exit of the radar beam,

$D=$ a dispersion effect which arises only from the broadening and consequent decrease in height of the signal pulse due to dielectric dispersion in the medium. An extended target (noncompact) may broaden the pulse. The radar antennas and components may disperse the pulse. Both of these effects are included in the FFT synthetic pulse calculations in section 5 and are not included in D,

$R$ = range, distance from radar antenna to target,

$R_{1}=$ the part of the range distance that is in the coal,

$C_{d}=$ convergence-divergence product to give the change of the signal $S$ due to refraction of the incident and the target returned signals through the interface.

In (4), the first fraction is the power density at the target and the second fraction is the returned power density at the radar, both in assumed free space. The third fraction is the receiving area of the radar. The terms $I, C_{d}, D, \exp \left(-4 \alpha R_{1}\right)$ represent various losses due to the ponderous medium and interface.

In the remaining discussion, the case of $R_{1} \neq R$ will be recognized only for the factors $D, C_{d}$, and $\exp \left(-4 \alpha R_{1}\right)$. 
Separation of the attenuation, $\exp \left(-4 \alpha R_{1}\right)$, from the dispersion effect, $D$, is an approximation. In section 5, these factors are taken into account by convolving the impulse response of the dispersive medium with transmitted and reflected pulses.

\subsection{Interface Transformation Factors, I $C_{d}$}

Factors of the interface problem are obtained by what is often called Sommerfeld's method for a dipole in a half space. Hufford, in 1969 [22], gave a rigorous derivation and practical results for the far field (obtained by geometrical optics) and for the ground wave, both in the air half space with a buried electric or magnetic dipole source. The treatise by Baños [34], is detailed; see especially his "Expansions valid over an entire hemisphere." Brekhovskikh's 1980 treatise [35] is useful because, with the source in either space, it gives specific equations for the geometrical optics term, the ground wave term, and a third term--the lateral wave. One of the authors of this report (HEB) has extended the geometrical optics solution to all ranges, from the interface to infinity in the refracted beam (as was necessary because the target was usually quite near the interface). Although our solution looks different, it agrees exactly with Brekhovskikh's first term.

Our solution will be briefly developed for an isotropic point source of power in a half space and assuming classical geometrical ray optics with energy conservation in the reflected and refracted ray-bundles (infinitesimal solid angles) of the problem--see figure 13. Finally, to get the radar factors I and $c_{d}$ of equation (4), the results must be applied twice. The point source in air transmits to the target which then transmits back to the radar.

The geometry of the problem in a spherical coordinate system is completely specified by assigning $z_{0}$, the perpendicular distance from the source to the interface, $n_{0}$ and $n_{1}$ the refractive indices of the source space and the other half space, respectively, and the location of the receiving point (field point) at a distance, $z_{1}$, from the interface and $\rho_{1}$ from the $z$ axis. From Snell's law, the ray paths are found giving the angles $\theta_{0}$ and $\theta_{1}$, and $r_{0}$ and $R_{1}$, the distances from the source to the point of refraction and from there to the field point, respectively. The source power is denoted as $w_{0}$ watts. The power density at distance $r_{0}$ is $s_{0}\left(r_{0}\right)=w_{0} /\left(4 \pi r_{0}^{2}\right) \quad W / m^{2}$ in homogeneous space.

Now consider the power transport by the rays in an infinitesimal solid angle, $d \Omega_{0}$, at incidence angle, $\theta_{0}$, that will refract to the target. The incident rays from the source are homocentric. Therefore, the power flow in the solid angle is $d W_{0}=s_{0}\left(r_{0}\right) r_{0}^{2} d \Omega_{0}$ at distance $r_{0}$ as it intersects the interface. Note that the E-field rms magnitude may be obtained from $s_{0}=E_{0}^{2} n_{0} Y_{0}$ where $Y_{0}$ is the wave admittance of the vacuum. The transmitted power of the refracted rays is the incident minus the reflected power. Thus, 
Fresnel's interface reflection coefficient, $\Gamma$, gives the power transmission coefficient, $\left(1-|\Gamma|^{2}\right)$. The power density, $s_{1}$, of the refracted beam based on energy conservation is expressed as

$$
s_{1}\left(R_{1}\right) d \sigma_{1}\left(R_{1}\right)=s_{0}\left(r_{0}\right) d \sigma_{0}\left(r_{0}\right)\left(1-|\Gamma|^{2}\right),
$$

where do is the area of a solid angle at the distance indicated.

It remains to find $d \sigma_{1}$ of the refracted beam. The solid angle of an incident beam element at angle $\theta_{0}$ may be written in spherical coordinates as

$$
\mathrm{d} \Omega_{0}=\sin \theta_{0} d \phi_{0} d \theta_{0},
$$

where $\phi=$ azimuthal angle and $\theta=$ polar angle.

For the refracted rays by symmetry, $d \phi_{1}=d \phi_{0}$ and by Snell's law

$$
d \theta_{1}=\left(n_{0} / n_{1}\right) \cos \theta_{0} d \theta_{0} / \cos \theta_{1}
$$

However, to find the area of the refracted beam, we must recognize the astigmatism of the refraction. At distance $R_{1}$ in the solid angle $d \Omega_{1}$, the range distance back to the apparent source of the ray bundle in medium 0 , as determined by tracing back two rays diverging by $d \phi_{1} \equiv d \phi_{0}$, is $R_{1}+r_{1}$ where

$$
r_{1}=r_{0} n_{1} / n_{0}
$$

(From this we find that the origin of the segment $r_{1}$ is a point on the $z$ axis.) Therefore, one dimension of the refracted solid angle, $d \Omega_{1}$, is

$$
\left(R_{1}+r_{1}\right) \sin \theta_{1} d \phi_{1}
$$

The range distance to an apparent source point as determined by tracing back two rays diverging by the angle $d \theta_{1}$ is $\left(R_{1}+r_{2}\right)$ where

$$
r_{2}=r_{1}\left(\cos \theta_{1} / \cos \theta_{0}\right)^{2} .
$$


The cross-section area of the refracted rays is then obtained at any distance, $R_{1}$

$$
d \sigma_{1}\left(R_{1}\right)=\left(R_{1}+r_{1}\right)\left(R_{1}+r_{2}\right) \sin \theta_{1} d \phi_{1} d \theta_{1} .
$$

Finally, substituting into (5), including Fresnel's $\Gamma$, we find the transinitted power density, $s_{1}$,

$$
\frac{s_{1}\left(R_{1}\right)}{s_{0}\left(r_{0}\right)}=\frac{r_{0}^{2}}{\left(R_{1}+r_{1}\right)\left(R_{1}+r_{2}\right)} \frac{n_{1}^{2} \cos \theta_{1}}{n_{0}^{2} \cos \theta_{0}} \frac{4 n_{0} n_{1} \cos \theta_{0} \cos \theta_{1}}{\left(n_{0} \cos \theta_{0}+n_{1} \cos \theta_{1}\right)^{2}}
$$

where TE denotes the TE mode, i.e., when the E-field is parallel to the interface. For the TM mode, replace the TE quantity by

$$
\left(n_{0} \cos \theta_{1}+n_{1} \cos \theta_{0}\right)^{2} \text { TM }
$$

Equation (7) may be evaluated at the interface $R_{1}=0$. It reduces to

$$
\left|E_{1}\left(R_{1} \rightarrow 0\right) / E_{0}\left(r_{0}\right)\right|=2 n_{0} \cos \theta_{0} /\left(n_{0} \cos \theta_{0} n_{1} \cos \theta_{1}\right)
$$

and, similarly, for the TN mode, both agreeing exactly with Fresnel's interface transmission coefficients (Stratton, 1941) [36].

We will show next that (7) reduces to Hufford's far-field solution as $R_{1}$ tends to $\infty$. First, we must renormalize (7) to Hufford's reference field, $E_{f}$. The field, $E_{f}$, is defined as the field that would exist at the receiver from the isotropic power source, $W_{0}$, if all space were homogeneously filled with the material having refractive index $n_{1}$,

$$
E_{f}^{2}(r)=w_{0} /\left(4 \pi r^{2} n_{1} Y_{0}\right)
$$

and $r$ is the direct distance from the source to the receiving point. The incident $E_{0}^{2}$ and $s_{0}$ have already been defined in terms of $W_{0}$ and $r_{0}$. After taking the square root of (7) with $R_{1}=\infty$, which inakes $r=\infty$, and replacing $E_{0}\left(r_{0}\right)$ by $E_{f}(r)\left(r / r_{0}\right)\left(n_{1} / n_{0}\right)^{1 / 2}$, we have

$$
\left|\frac{E_{1}\left(R_{1}\right)}{E_{f}(r)}\right|=\frac{2 n_{1}^{3 / 2} \cos \theta_{1}}{n_{0}^{1 / 2}\left(n_{0} \cos \theta_{0}+n_{1} \cos \theta_{1}\right)} \text {. }
$$


For the $M$ mode, use $\left(n_{0} \cos \theta_{1}+n_{1} \cos \theta_{0}\right)$ TM. Equation (8b) agrees exactly with Hufford's far-field result for his interface transmissions $\left|L C_{e}\right|$ and $\mid L$ $C_{m}$ l (TE and Tri) when specialized to his case; namely, $n_{1}=1$ in the air and $n_{0}$ is of the dense material in which the source is buried. Also, Hufford obtained $\left(\operatorname{Re} n_{0}\right)^{1 / 2}$ instead of our $n_{0} 1 / 2$ and he gave the phase of $E_{1} / E_{0}$. Having started with power densities, we lose the phase and must assume the dielectric loss tangents are less than 0.1 to yield square roots of $n_{0}$ and $n_{1}$ with less than 1\% error.

The following tabulation gives computer results for $\left|E_{1}\left(R_{1}\right) / E_{f}(r)\right|$ (found from equations (7) and (8a), valid at any value of $R_{1}$ consistent witn $z_{1}>\lambda_{1}$, the wavelength in medium (1), of figure 13, and also gives results for Brekhovskikh's $\Psi\left(R_{1}\right)$ multiplied by $r$--the distance defined in $E_{f}$, equation 8a. Reciprocal paths were calculated. The fields, $\Psi_{1}$, exhibit reciprocity as expected. The last column shows that $\left|E_{1} / E_{f} / \Psi\left(R_{1}\right)\right|=\left(n_{1} / n_{0}\right)^{1 / 2}$.

\begin{tabular}{cccccc}
$n_{0} / n_{1}$ & $\theta_{0}$ & $r_{0}, R_{1}$ & $\left|E_{1} / E_{f}\right|$ & $r \Psi\left(R_{1}\right)$ & $E_{1} /\left(E_{f} r \Psi\left(R_{1}\right)\right)$ \\
\hline 2 & $20.7^{\circ}$ & 1,1 & 0.546 & 0.7724 & 0.707 \\
$1 / 2$ & $45^{\circ}$ & 1,1 & 1.092 & 0.7724 & 1.414 \\
2 & $20.7^{\circ}$ & 5,1 & 0.788 & 1.115 & 0.707 \\
$1 / 2$ & $45^{\circ}$ & 1,5 & 1.577 & 1.115 & 1.414
\end{tabular}

The refraction contributes divergence when $n_{0} / n_{1}>1$ and convergence when $n_{0} / n_{1}<1$. Hufford's normalization is especially appropriate for practical calculation because $\left|E_{1} / E_{f}\right|^{2}$ is the power loss (or gain) of the interface. $\left|E_{1} / E_{f}\right|$ correctly changes value accordingly as divergence or convergence, $d$ or $c$, occurs. The separate factors $I$ and $C_{d}$ are given, respectively, by $\left(1-|\Gamma|^{2}\right)^{2}$ (which is the last fraction in (7) squared) and by the first two fractions in (7) evaluated in each direction and multiplied. The total interface radar power transfer is

$$
\text { I } C_{d}=\left|E_{1} / E_{f}\right|_{d}^{2}\left|E_{1} / E_{f}\right|_{c}^{2} \text {, }
$$

evaluating equation (7) first with subscript o denoting parameters in the denser medium and 1 denoting the rarer medium, convergent case, and then again with all parameters interchanged, diveryent case. For the above exainple, where $r_{0}$ and $R_{1}$ are 1,5 and then 5, 1 on return, the value of $I C_{d}$ is -1.545 , using the table. 
Summarizing, we have developed a convenient expression for Sommerfeld's geometrical optics term which is valid from the interface, $R_{1} \cong \lambda_{1}$ to $R_{1}=\infty$. The ground and lateral wave terms have not been included, a valid approximation when $\theta_{0}$ and $\theta_{1}$ are small and $z_{0}$ and $z_{1}$ are greater than $\lambda_{0}$ and $\lambda_{1}$ in the two media. However, when the source is at or on the interface, Engheta, [37] and Lewis [38] have demonstrated large correction of the optical term.

Our expression for this area in the refracted beam seems to be new, but agrees with Brekhovskikh. His area term is somewhat remeniscent of an equation in optics for the caustic which gives the location of the origin of our segment, $r_{2}$, cf. Martin and Welford [39]. Their equation is $a x^{2 / 3}+b y^{2 / 3}=1$ in a Cartesian system, where $a$ and $b$ are functions of $n_{0}, n_{1}$, and $z_{0}$.

Our caustics may be described as follows: The origin of $r_{1}$ is in medium 0 on the $z$-axis at a distance

$$
z_{1}=z_{0} \tan \theta_{0} / \tan \theta_{1}
$$

from the interface. The origin of $r_{2}$ is found by tracing the refracted ray at angle $\theta_{1}$ backward into medium 0 a distance

$$
r_{2}=r_{0}\left(n_{1} / n_{0}\right)\left(\cos \theta_{1} / \cos \theta_{0}\right)^{2}
$$

These equations hold no matter which medium contains the source.

\subsection{Attenuation in the Medium}

The term $\exp \left(-4 \alpha R_{1}\right)$ gives the power loss in the medium during the round trip. The factor $\alpha$ is the attenuation factor of the propagation constant in the medium,

$$
\alpha+j \beta=\left(-\omega^{2} \mu \varepsilon\right)^{1 / 2} \text {. }
$$

A good approximation for coal media at frequencies of interest is

$$
\alpha \cong 0.5 \beta \quad \varepsilon^{1 / 2} \tan \delta
$$

where $\tan \delta=\varepsilon^{\prime \prime} / \varepsilon^{\prime}$ for the medium, and $\beta_{0}=\omega\left(\mu_{0} \varepsilon_{0}\right)^{1 / 2}$, the phase factor of free space. The error of the approximation, (10), is proportional to 0.5 $\tan ^{2} \delta$ if $\tan \delta<0.1$ 
Section 5 uses exact complex scattering coefficients of a homogeneous cylinder to obtain exact synthetic pulse computer predictions of signatures of the various cylinders. Synthetic pulse trials also allowed the factor $D$ of eq (4) to be estimated by comparing the pulses in dispersive coal and fictitious nondispersive coal. From this we chose a very approximate value of $D=0.5$, for a range sufficiently large to bring $S / N$ down to approximately 2. (To a good approximation, $D$ is proportional to $d \varepsilon^{\prime} / d \omega$ in the radar frequency band, and to range $R_{1}$. Lynch [42] approximates $d \varepsilon^{\prime} / d \omega$ as proportional to tans.

\subsection{Radar Range Calculation from S/N}

The ultimate radar range may be defined by setting the signal power, $S$, of equation (4) equal to the noise power, $N$, of the radar receiver

$$
S / N=1
$$

and solving for $R$.

The noise power must be suitable for the type of radar, detection method, search method, time duration of display, etc. However, we will use the noise power often given for common of pulse radar,

$$
N=k T B N F
$$

where the Boltzman constant, $k=1.38 \times 10^{-23}$ watt $/ \mathrm{Hz} \mathrm{Kel}$ vin, $T=$ temperature = 290K, $B$ = receiving bandwidth in $\mathrm{Hz}$, and $\mathrm{NF}$ is the multiplicative noise figure. We have used $N F=6.31$, which is $8 \mathrm{~dB}$. This figure is somewhat higher than that for well designed systems where $4 \mathrm{~dB}$ to $5 \mathrm{~dB}$ might be attained. The noise figure theory is discussed in Mumford and Scheibe [23]. For baseband pulse radar, the $S / N$ may be obtained using the reflected pulse voltage, $S$, divided by the rms noise voltage, $N$, of the usual A-scope presentation. By signal processing, S/if can be enhanced by integration over many pulses with a sampling scope. The latter method may detect a signal smaller than noise, but may require a significant amount of time depending on the pulse repetition rate, pulse broadening by dispersion, and volume in the earth to be examined.

\subsection{Radar Cross Section}

The factor $\sigma$ is given by the equation (Bowman, Senior, and Uslenghi, equation I.30) [24],

$$
\lim (R \rightarrow \infty) \sigma\left|E_{i}\right|^{2}=4 \pi R^{2}\left|E_{s}\right|^{2},
$$


where $E_{i}$ is the field strength of the wave incident on the target and $E_{S}$ is the field strength of the scattered field at the receiving antenna. $R$ is the separation distance of the target and radar. $E_{s}$ must be either calculated or measured. Approximate calculations for a borehole and dielectric strip are given in sections 4.7 through 4.14 and used in Appendix $A$. (Exact cylinder scattering was used in section 5. )

The use of $\sigma$ in (4) is simply a way of stating the value of $\left|E_{s} / E_{j}\right|^{2}$ of the target. The use of $\sigma / 4 \pi R^{2}$ in (4) requires the $\sigma$ defined in (13). The so-called cylinder cross section, $\sigma^{c}$, cannot be used directly, even for a cylinder, in solving equation (4).

Values of $\sigma$ are often available based on the theory of a plane wave, the source at infinity. As will be discussed, we deduce correction factors of 0.5 and 0.25 to the theoretical $\sigma$ of infinite cylinders and an infinite plane, respectively, when the source is at a finite distance as for practical radar.

\subsection{Electromagnetic Theory of Scattering Cross Sections of Extended Targets}

The radar cross section in (4) and (13) may be essentially constant, i.e., independent of range, $R$, for compact targets. Then, e.g., $\sigma$ of a large metal sphere is $\pi r^{2}, r=$ sphere radius $R \gg r>\lambda$. For a general convex, large, compact metal body

$$
\sigma=\pi r_{1} r_{2},
$$

where $r_{1}$ and $r_{2}$ are the two radi $i$ of curvature at the reflection point.

For extended bodies, however, the cross section is usually a function of the range distance, $R$. For example, $\sigma$ of an effectively infinite planar metallic interface is $\pi R^{2}$. With this cross section, the signal in (4) decays as $R^{-2}$, instead of $R^{-4}$ as for compact targets. Cook [19] deduced $R^{-3}$ dependence, qualifying it as being only for a rough surface.

The references on radar cross sections, (Berkowitz, p. 556) [20], (Kerr, sect. 6.2) [25], (Ruck) [26], and the basic EM scattering treatises, e.g., (Bowman, Senior and Uslenghi) [24], do not specifically give these rangedependent cross sections. The basic theory is the basis for the cross sections that we will give for cylinders representing boreholes, and for strips representing old workings tunnels. The purpose here will be to obtain theoretical values for such targets, including the necessary range dependence. Tarantolo and Unterberger [27] deduced a dependence on range, beamwidth, and pulse length. Our results differ from theirs. In particular, the beamwidth is not a necessary parameter for usual conditions. 
Consider, as a simple example, a small radar antenna interrogating an infinitely extended flat metallic interface. The field is $E_{0}$ at $1 \mathrm{~m}$ from the source point. The incident field at the target at distance $R$ is then

$$
E_{i}=E_{0} / R \text {. }
$$

By image theory, the radar receiving antenna may be at distance $2 R$ with the interface removed. Therefore, we deduce that the received (scattered) field is

$$
E_{S}=E_{j} / 2 \equiv E_{0} / 2 R \text {. }
$$

These fields in eq (13) give

$$
\sigma=\pi R^{2}
$$

dependent on range as was stated previously.

\subsection{Half-period (Fresnel) Zones}

Radar aspects of scattering from a plane, a strip, and a cylinder may be obtained by Kirchhoff-Fresnel-Huygens' concepts of wave surfaces and scattering surfaces as intermediate sources. There are half-period (Fresnel) zone areas on the surfaces that contribute a range of phases from zero to $\pi$, $\pi$ to $2 \pi$, and so on. Kerr [25], section 6.2, obtained radar cross sections of plane and curved surfaces by the vector Kirchhoff-Huygens method. He states, $p$. 417, that Fresnel zones may be defined on reflecting surfaces.

The scalar Kirchhoff-Huygens method will be used here to obtain an approximate solution for a strip, and to obtain a rather good estimate of the difference between theoretical plane wave incidence, and practical pointsource, radar cross sections. First the planar surface will be reconsidered and (14) redeveloped from diffraction theory to illustrate the Kirchhoff method.

In a Cartesion coordinate system, consider a planar reflecting intermediate surface lying in the $y z-p l a n e$ at $x=0$ with a point receiver at $x=R$ and a point source at $x=R^{i}$, both on the $x$-axis. The radius of the circular first half-period zone on the intermediate surface is

$$
r_{1}=\sqrt{R^{\top} R \lambda /\left(R^{1}+R\right)}
$$

assuming $R, R^{\prime} \gg \lambda$. In the case of monostatic radar $R^{\prime}=R$, 


$$
r_{1}=\sqrt{R \lambda / 2} \text {. }
$$

In the case of a fictitious plane wave incident radar, $R^{\prime}=\infty$,

$$
r_{1}=\sqrt{R \lambda} \text {. }
$$

There are successive annular half-period zones surrounding the first zone. For more discussion see, e.g., Jenkins and White [31], chapter 18, and Kerr [25], chapter 5 .

The total reflected field from a smooth flat or curved intermediate surface is almost proportional to the area of the first zone. For a planar target, the areas and consequent relative scattered fields, $E_{s} / E_{j}$, for monostatic and for bistatic plane wave incident radar are proportional to the circle areas, $\pi R \lambda / 2$ and $\pi R \lambda$, respectively, from $(15 b, 15 c)$. The scattered fields proportional to these areas are (by a calculation similar to Fresnel's integrals in equation (17))

$$
\begin{aligned}
E_{s, m} & =E_{i} / 2, \\
E_{s, p w i} & =E_{i},
\end{aligned}
$$

where $m$ denotes monostatic, and pwi denotes plane wave incident radar.

The interesting conclusion may be drawn that the concept of half-period zones enables us to solve, approximately, the problem of how to obtain monostatic point-source radar cross sections from known canonical plane wave incident scattering solutions, as will be further demonstrated below.

For the radar interrogation of an infinite planar interface, equations (16a) in (13) give

$$
\begin{aligned}
\sigma_{m} & =\pi R^{2} \\
\sigma_{p w i} & =4 \pi R^{2} .
\end{aligned}
$$

$\sigma_{m}$ here correctly agrees with equation (14). 
Consider next an infinitely long dielectric strip representing one side wall of an old workings tunnel. The reflection of the farther side wall of the tunnel will be discussed qualitatively later. Assume that the width of the strip is $1.5 \mathrm{~m}$ (5 feet), which equals 1 to 5 wavelengths in air (more in coal), for radar whose main spectrum is in the band $0.2 \mathrm{GHz}$ to $1.0 \mathrm{GHz}$. With these parameters, the reflection coefficient of the assumed strip may be represented by a physical optics approximation, which means that the discontinuities at the edges of the strip are disregarded.

It is useful to discuss a metallic strip initially instead of the dielectric interface strip. When the range, $R$, to the radar (which is located on a normal from the center of the strip) is of the order of the strip width, $2 \mathrm{w}$ $(\sim 1.5 \mathrm{~m})$, of the order of a few wavelengtins or less, the cross section is approximately that of an infinite planar interface, i.e., $\sigma \sim \pi R^{2}$, from (16b). The reflection will be relatively strong at such a close distance. It may be shown by means of Fresnel integrals that $\sigma$ will vary by about $\pm 2.5 \mathrm{~dB}$ from $\pi k^{2}$, depending on $w / r_{1}(w=$ half width of strip).

The important case of the strip far from the radar, presenting a small cross section, may be solved by application of the Kirchhoff-Huygens method. Assume the width, $2 w>\lambda$, so that the discontinuities at the edges of the strip may be neglected, and the range, $R \gg w$, so that the waves are approximately at perpendicular incidence over the first Fresnel zone. The results for $\mathrm{plane}$ wave incident (pwi) radar and for monostatic radar look the same; E-scattered is

$$
E_{s}=E_{i} \frac{(1+j)}{\lambda R} r_{1}^{2}[C(u)-j S(u)]
$$

where $E_{j}$ is incident field, $C$ and $S$ are Fresnel's cosine and sine integrals (tabulated functions) and $u=\sqrt{2} w / r_{1}$. (The total phase lag of $R^{\prime}+R$ is suppressed.) For pwi radar $R^{\prime} \rightarrow \infty$ and $(15 \mathrm{C})$ is used. For monostatic radar $R^{\prime}=R$ and $(15 b)$ is used. For the conditions assuned, especially $R \gg w$ which gives $r_{1} \geqslant 2 w$, the approximations $c(u)=\sqrt{2} w / r_{1}$ and $S(u)=0$ hold, giving

$$
\begin{aligned}
\left|E_{s} / E_{i}\right|_{m} & =\sqrt{2} w / \sqrt{\lambda R} \text { (monostatic), } \\
\left|E_{s} / E_{i}\right|_{p w i} & =2 w / \sqrt{\lambda R}(p w i) .
\end{aligned}
$$

These field ratios in (13) give range-dependent cross sections,

$$
\begin{gathered}
\sigma_{m}=8 \pi R w^{2} / \lambda \equiv 4 k_{0} w^{2} R, \\
\sigma_{p w i}=16 \pi R w^{2} / \lambda \equiv 8 k_{0} w^{2} R .
\end{gathered}
$$


It may be confirmed that these results are a good approximation by suitable comparison with eq $7.4-17$ (Fig. 7-17) of Ruck [26], which gives

$$
k_{0} \sigma^{c} \sim 1+\left(2 k_{0} w\right)^{2}
$$

where $\sigma^{C}$ is the cylindrical cross section per unit length, not a function of range, $R$. The cylindrical cross section in (20a) is defined as $\sigma^{C}=1$ im $2 \pi R$ $\left|E_{S} / E_{i}\right|^{2}$, in the limit as $R \rightarrow \infty$. (As was discussed, the correct $\sigma$ radar is obtained by putting $E_{S} / E_{j}$ in equation (13)). However, for the present comparison, we want $\sigma^{C}$ (from (18b)) which is obtained as

$$
\begin{aligned}
\sigma^{c} & =8 \pi w^{2} / \lambda, \\
k_{0} \sigma^{c} & =16 \pi^{2} w^{2} / \lambda^{2} \equiv\left(2 k_{0} w\right)^{2},
\end{aligned}
$$

almost agreeing with Ruck, $i . e .$, with (20a) but missing the 1 of (20a) because we have neglected edge effects, which is valid because $w / \lambda>1$. It is concluded that our range-dependent cross sections (19a) and (19b) are correct approximations, neglecting edge effects.

\subsection{Cross Section of a Dielectric Tunnel at Broadside Incidence}

The necessary next approximation is to assume that the cross section of a dielectric strip interface may be obtained from that of a metal strip by the relation

$$
\sigma_{\text {diel }}=\sigma_{\text {metal }}|\Gamma|^{2}
$$

where $\Gamma$ is the reflection coefficient at the tunnel interface. Using the notation refractive index, $n=\varepsilon^{\star^{1 / 2}}$, the reflection coefficient in passing from medium 1 to medium 2 is

$$
\Gamma=\left(n_{1}-n_{2}\right) /\left(n_{1}+n_{2}\right)
$$

For example, $\Gamma$ from coal with $\varepsilon_{1}=5$ and a water-filled shaft, $\varepsilon_{2}=81$, is

$$
\Gamma_{W}=-0.60
$$


and from coal to a gas such as methane or air is

$$
\Gamma_{\mathrm{a}}=+0.38
$$

These values in (21) show that $\sigma$ of a water-filled shaft is 2.5 times greater than for a gas, depending upon the assumed permittivity of the coal medium. The farther wall of a tunnel has a reflection coefficient of the same magnitude as the first wall. There are multiple reflections in the time domain. However, the EM power returned by the second wall is

$$
\left(1-|\Gamma|^{2}\right)^{2}
$$

weaker than from the first wall, due to the transmission loss in each direction at the first wal1. (Equation (22) is the square of the factor in (5).) These multiple reflections will be seen as successively smaller reflections in the time domain, or as interfering varied amplitude returns with an FM-CW swept radar.

We note that, with gas in a shaft, the sign of the reflection coefficient at the first wall is positive. With water, the sign is negative. The opposite occurs at the second wall. In effect, this is also true for detecting a borehole when $k_{0} a n_{2}$ is large.

We conclude that, at least theoretically, the radar return signature may be used to identify the target, i.e., to solve the inverse problem. The magnitude and sign of the reflection coefficient, and/or the rate of decay of successive reflections (influenced by attenuation of water) may give the width of the shaft and distinguish between water and gas filling. Successive reflections are seen in the simulated pulse study in section 5.2. Thus, detailed data could contribute to solving the inverse problem. Wittmann [40] has demonstrated comparable synthetic pulses in a planar layered medium. Roe and Wittmann [41] gave a synthetic analysis of the phasor signals of the FM-CW radar scattering from a planar layered medium, to distinguish $n_{1}<$ or $>n_{2}$.

\subsection{Cross Sections of Circular Cylinders (Boreholes)}

The radar scattering of circular cylinders involves concepts similar to those brought out in the scattering of a strip (tunnel). Just as for the strip, we will convert to the monostatic radar case by observing that the significant excited length along the cylinder is 0.707 of that for the canonical plane wave incidence case.

The scattering of a small cylinder is much greater with TM polarization than with TE polarization. Only the TM case will be discussed. In the mine, the antennas should be oriented to give a vertical E-field. However, it would be useful to rotate them by $90^{\circ}$ in trying to solve the inverse problem of identifying a target, e.g., to distinguish a borehole from a tunnel and to identify clutter. 
The scattering from a metallic cylinder with plane wave incidence and the receiving point at distance $p \gg a_{,} a=c y l i n d e r$ radius, is

$$
\begin{aligned}
& \lim (\rho \rightarrow \infty) E_{S}=E_{i}(2 / \pi k \rho)^{1 / 2} P, \\
& P=-\sum_{n=0}^{\infty}(-1)^{n} e_{n} J_{n}(k a) \cos n \phi / H_{n}^{(2)}(k a)
\end{aligned}
$$

where $e_{n}=1$ for $n=0$ and $e_{n}=2$ otherwise, and

$$
k=\left(\omega^{2} \mu_{0} \varepsilon_{0} \varepsilon^{*}\right)^{1 / 2}
$$

The $J_{n}$ are Bessel functions, $\phi=0$ for monostatic radar, and the $H_{n}^{(2)}$ are Hankel functions for outgoing waves, section 2.2.1 [23], [26]. In (23) the phase factor, $\exp (-j k \rho-j \pi / 4)$, has been omitted. The $\varepsilon^{*}$ is of the surrounding medium.

Using $E_{s} / E_{j}$ of equation (23) in (13), which amounts to assuming that there is a bistatic radar with the source at infinity and the receiver at $\mathrm{R}=$ $p$, we get

$$
\sigma_{p w i}=8 \mathrm{R}|\mathrm{P}|^{2} / \mathrm{k}
$$

However, for practical monostatic radar the $\sqrt{2}$ is removed from the numerator of (23) giving

$$
\sigma_{m}=4 R|P|^{2} / k
$$

As in equation (16) for the strip, $\sigma_{m}=0.5 \sigma_{p w i}$. The $k$ is in the medium. It may be shown that in the geometrical optics approximation, $i . e ., a>\lambda$, $|P|^{2}=k \pi a / 4$, which in (27) gives

$$
\sigma_{m, G .0 .} \sim R \pi \mathrm{a} .
$$

The dependence of cross section on range, $R$, was also demonstrated in the development of equations (14) and $(19 a, b)$. The change in cross section for the case of plane wave incident radar and monostatic radar, a factor of 4 in (17b) and factor of 2 in (19a, b), was deduced. The reasons for all of these results may be based on the concept of the first Fresnel zone area as a function of $R$ and the consequence that $\sigma_{m}=0.5 \sigma_{p w i}$ for a long cylinder. 
The method of obtaining the monostatic cross sections of strips and cylinders by multiplying $\sigma_{p w i}$, by 0.5 furnishes a very simple approximation which avoids difficult calculations for a full wave [28], point source radar solution in place of eq (23). Our approximate results are, however, believed to be reasonably accurate for $R \gg a, R \gg \lambda$. (We recently found that Brysk [32] deduced the factor $0.5=\sigma_{\mathrm{m}} / \sigma_{\text {pwi }}$ and a range dependent $\sigma_{.}$)

\subsection{Computer Routine for S/N Versus Range}

The appendix lists a computer program for calculating the signal/noise ratio, S/N, of an FM-CW radar. The program is in BASIC language and is suitable for a desk top computer. It solves for $S$ of (4) divided by $N$ of (12) as a function of range, $R$. The approximation of equations (21) and (22) are reasonable for large water-filled bore holes; but for air filling, the ranges are too optimistic--especially if the hole diameter is less than the wave length in the coal. (For the tunnel, (21) and (22) are good approximations.)

Figure 14 shows curves of $S / N$ as a function of distance for detecting a $30-c m$ diameter well. Reasonable values for two coal "atmospheres" and three borehole contents: metal, water, and gas are assumed.

The exact solution (23) is used for metallic cylinders in the intermediate range, $\mathrm{ka}$, of the order of 1 . For small ka, an accurate Rayleigh-type approximation is used. For large ka, the geometrical optics value, $\sigma^{c}=\pi a$, as modified (28) for monostatic radar, is used. The cross sections with dielectric filling were assumed to be the above multiplied by $|\Gamma|^{2}$, equation (21).

\subsection{Conclusions}

The radar signal equation was modified to take into account changes in the signal due to the coal "atmosphere". These effects include mainly the air-to-coal interface, the attenuation of the waves by the dielectric loss, and the spreading of the pulse by dielectric dispersion in the coal. The last effect had to be estimated; more research on dispersion could be useful.

Regarding the target locations, they were idealized as being straight ahead of the working antennas. A borehole off to the side would, theoretically, give a weaker signal because the radar antenna gives maximum gain straight ahead. Preliminary experiments to evaluate the degradation of the range capability for off-side boreholes would be useful.

The old tunnel may give a small cross section if it is higher or lower than the radar antennas and, also, when it is not perpendicular to the working tunnel surface. Regarding the relative heights, the inevitable roughness of the old tunnel wall will furnish some non-specular reflections, thus permitting some reflection, though weak compared to the specular reflection cross 
section used in (19a). For non-perpendicular intersection of the old and new tunnels, the antenna gain pattern, $G_{0}$, of the radar antennas for the ray angle that arrives at perpendicular incidence on the old tunnel will determine the change in the cross section.

There is a real need for experience with clutter. The scattering of various clutter targets both attenuates the wave to and from the desired target and gives interfering signals that may, in effect, raise the noise figure, NF, of (13).

It may be noted that Ruck [26], devoted Chapter 9 to rough surface effects. Their work could be useful in extending the present work.

We have attempted to give a clear explanation of the cross sections of infinite planes, cylinders, and strips. Some of our results may be new. The modification of plane wave scattering theory for point-source radar scattering was investigated and solved. To find $\sigma$ for eq (4) from $\sigma^{c}$ use $\sigma=2 R \sigma^{c}$.

Finally, it seems necessary to caution that with the targets off-side, higher and lower, walls not perpendicular, rough targets, and also clutter, we cannot expect ranges to be as great as those calculated in the figures.

The antenna pattern in the material is limited to forward directions. For example, if the pattern in air covered the whole forward hemisphere, then the pattern in coal with $\varepsilon^{\prime}=9$ would cover only $19.5^{\circ}$ from straight ahead of the significantly illuminated area of the interface.

\section{Pulse Response Simulations}

Impulsive signals have typically been used at NBS for geophysical probing because they are relatively easy to generate at high repetition rates, and they have a broad spectral content and a short duration which permits discrimination between closely-spaced returns. On the negative side, it's very difficult to construct efficient, portable antennas with sufficient bandwidth. In addition, the higher frequency components of the 1/2-ns NBS impulse generator are severely attenuated in lossy materials such as coal.

At the contract monitor's suggestion, a comparison of potentially useful pulse waveforms was done by simulating the transmission between the two TQ antennas in air. The received signals are examined for amplitude, width, distortion, and for the feasibility of using additional signal-processing techniques. The analysis is extended to include transmission through a lossy medium and reflection from a borehole as described in chapter 4 . The result is an estimate of the likelihood of detecting a borehole at a given range with an actual measurement system. 
The two TQ antennas, separated by $1.5 \mathrm{~m}$, are treated as a two-port network. The simulated response of this network to any signal is the convolution of the input waveform with the measured network impulse response. Impulses of 1-volt amplitude and 1/2-, 1-, 2-, and 5-ns duration at the 50\% point were approximated by the raised cosines shown in figure 15 with the spectral distributions of figure 16. Clearly, the 5-ns impulse has so little energy in the pass band of the antennas (see figure 3) that only a very sinall signal would be expected at the receiving antenna terminals. The results of the convolution operation (figure 17) confirm that impulses with a duration of inuch inore than 2 ns will be too inefficiently transmitted to be useful with the TQ antennas.

Similar comparisons using 1 volt peak-to-peak sinusoids of 1,2 , 4 , and 8 cycles are illustrated in figures 18 through 20 . The carrier frequency was chosen as $250 \mathrm{MHz}$ to approximate the transition duration of the impulse generator to be delivered to the Bureau of Mines as a part of this contract. The monocycle, with its reduced energy at lower frequencies, may be an improvement over the impulse. The received signal is no better for the other three 250 $\mathrm{MHz}$ examples, and the additional pulse width would make detection of adjacent geologic features more difficult.

Above-ground radar systems use pulse compression schemes to combine a narrow response with higher average power. This can be accomplished by transmitting a carrier frequency which is a linear function of time, a so-called chirp, and cross correlating the returns with a replica of the chirp. Hardware is becoming available which could potentially perform the same function quickly in a coal mine environment without extensive computer processing.

A set of linear chirp waveforms are shown in figure 21. These were chosen as an estimate of what might be usefully transmitted using the TQ antennas. The three waveforms are: 250-500 $\mathrm{MHz}$ in $10 \mathrm{~ns}, 250-1000 \mathrm{MHz}$ in 10 $\mathrm{ns}$, and 250-1000 $\mathrm{MHz}$ in $20 \mathrm{~ns}$. The spectra of these pulses are shown in figure 22. The resulting received waveforms, after transmission over a $1.5 \mathrm{~m}$ path with a pair of TQ antennas, are shown in figure 23. Correlation techniques would be less effectively applied to the upper waveform which shows little evidence of the frequency change. The higher frequencies of the other two waveforins would be severely attenuated in the coal. That additional distortion could limit the system's effectiveness. A fourth chirp was numerically generated covering 300-600 $\mathrm{MHz}$ with a 20-ns duration. Figure 24 shows that the simulated received waveform retains the chirp characteristic. The autocorrelation of that waveform as depicted in figure 25 demonstrates the extent of the pulse compression possible. Compare this result to the autocorrelation of the 4-cycle, $250 \mathrm{MHz}$ sine wave presented in figure 26. Both received waveforins are about 25 ns in duration, but the chirp has a much narrower autocorrelation function.

Multi-cycle sinusoids will be less effective sources than will simpler waveforins until rapid coding schemes can be perfected. Chirps, on the other hand, offer distinct advantages if the "clutter" signals can be suppressed. Some additional work is certainly warranted in this area. At present, it seems that 1 - to 2-ns wide impulses or monocycles are optimal choices for the antennas and detection system. 


\subsection{Borehole Detection Estimates}

It is helpful to have a method which predicts the response of a measurement system to a particular mining threat. One can combine an incident waveform with the antenna impulse response as was done in the previous section, correct for the attenuation in a lossy dielectric material, include the reflection characteristics of a given target, and thereby estimate the amplitude and form of the received signal. The analysis can predict the ability of a given system and data processing technique to discriminate between targets and background responses and can estimate the limiting range of the system.

A 1-volt amplitude, 1-ns duration impulse (figure 15) was chosen as the incident waveform and convolved with the TQ antenna impulse response. The antennas were assumed to be immersed in the dielectric medium, i.e., no corrections were made for the air/coal interface in the simulation. The antennas might well be placed directly against the work face when the system is to achieve maximum range. Typical surface roughness and contours add great uncertainties to any estimate of the reflection at that interface.

The coal seam was modeled as a frequency independent dielectric with infinite extent. Dry coal was assigned a relative dielectric constant of 3.65 and a loss tangent of 0.03 ; for damp coal, it was 6.5 and 0.05 , respectively. These are reasonable approximations since our primary operating range lies above $200 \mathrm{MHz}$. When measured values of dry coal permittivity versus frequency were substituted for the frequency independent value, the results were not substantially changed. We are not aware of measurements on damp coal covering a broad enough spectrum for this analysis.

Vertical boreholes representing the uncharted, abandoned wells are the mining threats of greatest interest for this work. Based on Bowman [24], the complex reflection coefficient was computed at each of the component frequencies of the simulated pulse for several types of boreholes in dry and damp coal. The antennas are considered as point sources. The responses to a metal-cased 15-cm radius borehole, an empty hole, and one that is water-filled are given in figure 27 for a $1-m$ range. As expected, the response from the cased hole is larger and inverted when compared to the empty hole return. The water-filled, uncased pattern is much more complicated. The slightly saline solution delays the signal enough that each reflection across the diameter of the pipe is distinct and inverted from the previous one. Thus the sign, amplitude, and pattern of a response may well indicate the type of threat encountered. Figure 28 illustrates the rapid attenuation of the response to a cased $15-\mathrm{cm}$ radius borehole in relatively damp coal as the target range is increased. The received signal drops by more than $30 \mathrm{~dB}$ as the range is increased from $2 \mathrm{~m}$ to $5 \mathrm{~m}$.

A summary of these simulations is presented in figures 29 and 30 . The predicted responses from five types of boreholes at ranges to $10 \mathrm{~m}$ in dry and damp coal are shown. Since the noise introduced by the sampling circuitry of a time domain receiver is on the order of $1 \mathrm{mV}$, high voltage pulses, preamplification, and signal averaging will be required if the system is to recognize boreholes at even a five meter range. Electromagnetic (EM) techniques have been used over large ranges in salt domes. The response of the 
systell to a $15-\mathrm{cm}$ borehole in rock salt at various ranges is also shown on figure 29. A relative dielectric constant of 6.5 and a loss tangent of 0.001 was assumed for rock salt. Clearly, operation at much larger ranges is possible in a low loss material such as salt.

\section{Pulse System Equipment}

A prototype time domain measurement system has been assembled for delivery to the Bureau of Mines at the conclusion of Phase II. The design goals emphasized a portable system that could be readily interfaced to the existing equipment at both the Bureau of Mines and NBS and which offered flexibility and fast waveform acquisition. As shown in figure 31 , the equipment includes the TQ antennas described in chapter 2, and an impulse generator and equivalent-time sampler located at the antennas. A two-channel fiber-optic link carries the analog signal and trigger to a remotely-located digital acquisition unit where the waveforms are analyzed and stored on floppy disks. In this arrangement, laboratory equipment has been integrated into a wide bandwidth system useful for a variety of subsurface measurements.

\subsection{High-voltage Impulse Generator}

A specially constructed pulser supplies 500-volt high, $\sim 2$-ns wide impulses to the 50-ohn transmitting antenna at a pulse repetition frequency (prf) of $10 \mathrm{kHz}$ (see figure 32). The spectral content of the pulse overlaps the operating ranges of the TQ's and other Bureau of Mines' antennas. A stable pretrigger, adjustable from $50 \mathrm{~ns}$ to $300 \mathrm{~ns}$, is furnished to the highfrequency sampler. The unit may be operated from either the power line or a 12-volt battery. Always set the 10-turn voltage cuntrol to 0 (full counterclockwise) and the RATE switch to OFF before turning the power ON. Allow a two-minute warm up, set RATE to ON to enable the clock circuitry and pretrigger, and increase the 10-turn voltage control. Set that control to 0 again before turning the pulser OFF.

\subsection{Receiver Preamplifier}

An optional 29-dB amplifier between the receiving antenna and the highfrequency sampler provides two functions in the system. It boosts low-level signals of up to $30 \mathrm{mV}$. The amplifier has good fidelity and a bandwidth of $10 \mathrm{MHz}$ to $2 \mathrm{GHz}$. In addition, it acts as a limiter for signals of up to $1 / 2$ volt at its input, holding the output to about 1 volt maximum. The amplifier compresses the signals from the receiving antenna, enabling the sampler to recover returns which otherwise would be buried in the sampling noise while protecting the sampling diodes from dainaging voltages. The unit has a quick recovery from saturation. The input to the amplifier must be limited to not more than 7 volts for 3 ns or the unit may be darnaged. When using the highvoltage impulse generator, take care that the peak received signal (usually from the air/coal interface) does not exceed $1 / 2$ volt. Add attenuators and/or renove the amplifier when large signals are likely. 


\subsection{High-frequency Sampler}

The sampler is used to construct a discrete, equivalent-tine replica of the repetitive signal from the receiving antenna/preamplifier. By sequentially acquiring one point per repetition at increasingly delayed times over 1024 signals, the system assembles a waveform which resembles the input signal but with a duration of about $100 \mathrm{~ms}$ instead of $200 \mathrm{~ns}$. This Tonger waveform can easily be digitized in real time and transferred to a computer for further processing.

The high-frequency sampler is a modified version of a commercial oscilloscope plug-in and is compatible with the present equipment at both NBS and the Bureau of Mines. The unit uses replaceable sampling heads which offers several advantages. First, if a sampling diode bridge were damaged on a field measurement, the head alone could be exchanged; there would be no need to send the modified sampling plug-in for repair. Second, a higher performance sampling head could be substituted if wider bandwidth signals were to be measured. The sampling head furnished with this system covers dc to $1 \mathrm{GHz}$ at - $3 \mathrm{~dB}$, but models are available with responses to $12 \mathrm{GHz}$. Finally, the sampling head is quite small, and possibly a long extender cable could be integrated into the sampler in the future to allow the head to be lowered into a borehole. This potential was attractive to the Bureau of Mines. The normal input range of the sampler is up to 1 volt peak-to-peak with a tangential noise of $1 \mathrm{mV}$, indicating a dynamic range of $54 \mathrm{~dB}$ without signal averaging.

The high-frequency sampler could be set up as follows for a coal-seam measurement:

$\begin{array}{lll}\text { TIME-DISTANCE } & : & 0, \times 1 \\ \text { TIME/DIV } & \vdots & 20 \mathrm{~ns} \\ \text { PRESET } & \text { Out } \\ \text { HIGH RESOLUTION }: & \text { Out } \\ \text { inV } & \vdots & \text { In } \\ \text { ATTENUATOR } & \vdots & 200 \mathrm{mV} / \text { diV } \\ \text { STABILITY } & \vdots & \mathrm{CCW} \\ \text { LEVEL } & \vdots & \mathrm{CW} \\ \text { SLOPE } & : & +\end{array}$

With that arrangement, a 200-ns time window would be acquired which represents a 10- to 15-m range. Since the time corresponding to the left edge of the window can be varied froin 0 ns to 1000 ns, the total observable range is from $0 \mathrm{~m}$ to between $60 \mathrm{~m}$ and $90 \mathrm{~m}$, depending on the permittivity of the coal. If shorter ranges are desired, set the time-distance dial to X.1, which permits a viewable range in damp coal of at least $10 \mathrm{~m}$ from the face.

The output of the sampling process is a discrete waveform resembling a staircase in which each step corresponds to a single sanple. The frequency at which the samples are taken is constant regardless of the time window selected and is equal to the prf of the inpulse generator. The SCAN control must be adjusted to set the width of the equivalent-time waveform so that enough samples are included to accurately characterize the sampler input signal. At 
least 5 samples/nanosecond are required for the high-voltage impulse generator. The product of that number, the TIME/DIV setting, and 10 divisions yields the total number of samples in the equivalent-time waveform--in this case, 1000. The duration of that waveform is the number of samples divided by the inpulse generator pulse repetition frequency, $10 \mathrm{kHz}$ for this case. Thus, the impulse generator prf (or $30 \mathrm{kHz}$, whichever is less) dictates the rate at which samples are taken, the scan control determines the number of samples in the equivalent-time waveform, and the TIME/DIV setting maps the real time window into equivalent-time. At full clockwise, the SCAN control selects about 512 samples. That number increases as the knob is turned counterclockwise. Connect an oscilloscope to the SWEEP OUT jack, and adjust the SCAN control until the ramp is the correct duration.

The plug-in "blanking" signal provides the trigger marking the start of the equivalent-time waveform. The output waveform is derived from the plug-in "VERT SIG OUT" which is scaled to 1 volt peak-to-peak to be compatible with the fiber-optic system. The high-frequency sampler may be connected to the digital acquisition system through the fiber-optic link or through up to $20 \mathrm{~m}$ of coaxial cable by reconnecting two coaxial cables within the sampler. The plug-in may also be removed from the sampler and inserted directly in an oscilloscope for laboratory use.

\subsection{Fiber-optic Transmission System}

A two-channel, fiber-optic transmission systen provides low noise signal paths from the high-frequency sampler, which is located at the receiving antenna, to the digital acquisition system at a remote point. The system consists of two channels, one used to transinit the equivalent-time replica of the received signal and the second for a trigger marking the start of that waveform. Each path consists of a transmitter, approximately $125 \mathrm{~m}$ of fiberoptic cable, and a receiver. The system has a maximum signal, in or out, of 1 volt peak-to-peak and a frequency response of $15 \mathrm{~Hz}$ to $25 \mathrm{MHz}$. The dynamic range has been verified at greater than $50 \mathrm{~dB}$.

Both commercial transmitters have been modified. The input impedances have been increased to 600 ohins to reduce the loading of the previous stages. In addition, an R-C differentiating circuit was added to the transmitter in the trigger path to condition the TTL "blanking" signal to the bandwidth and input voltage limits of the fiber-optic system. As a result, the transinitter units are not interchangeable and damage may result if the cables to the transmitters are reversed.

The receiver units require 50-ohm terminations. A feedthrough terminator must be used on the signal path. The trigger signal is terminated and transformed to $>2$ volts to reliably trigger the digital acquisition systeln. No additional termination is required for that channel. 


\subsection{Digital Acquisition System}

The digital acquistion systein consists of a microprocessor-controlled waveform recorder, monitor, and 5 1/4-inch floppy disk drive. The waveform recorder can acquire up to 10,000 points at a $66 \mathrm{kHz}$ rate with 12-bit $\mathrm{A} / \mathrm{D}$ conversion. It can average repetitive waveforms to reduce the effects of random noise, correct for time and amplitude scaling, apply time-weighted-gain functions, and subtract previously acquired waveforms such as background or clutter waveforms. The measurements, setups, and procedures are stored on diskettes or transferred to a controller/computer via either RS-232 or GPIB interfaces.

The unit would nomaliy be used in the averaging mode, external trigger at $10 \%$ level, with the sweep time equal to the duration of the high-frequency sampler's equivalent-time waveform, and with 1000 element arrays.

\section{FIELD TESTING}

\subsection{NBS Test Range}

An indoor test range has been installed at NBS (see figure 33 ). This test range holds both air-filled and water-filled voids for use in checking underground void measurement or imaging systems. The loss in the soil of the range goes from about $25 \mathrm{~dB}$ per meter at $300 \mathrm{AHz}$ to about $50 \mathrm{~dB}$ per meter at $1 \mathrm{GHz}$. This loss is quite high for operation of any radar systern at these frequencies and thus represents a rather stringent test of any detection system.

Several series of measurements were taken with the pulse and FM-CW systems using different antennas and antenna arrangements. Using the TQ antennas with a center baffle (as described in section 2.4), a sequence of 26 measurements was made starting at the end of the range having the air-filled targets. The transinitting and receiving antennas with the septum were moved along the range as an assembly. Responses were recorded at 5-cm intervals. Figure 34 shows a typical result using a possible data handling method. Curve 1 shows the average background response for the series. Each point on that curve is the mean of the 26 responses at that relative time. Curve 2 shows one of the response curves taken over the $0.5-m$ deep target. Curve 3 is obtained by subtracting an average background from a response taken over a target to show how the target signal may be enhanced. The target response is shown by the arrow in figure 34. A series of enhanced responses is shown stacked in figure 35. The hyperbolic contour of the target signal (arrow) can be relatively easily seen.

Other approaches to the deconvolution problen were tried, but at this time no significant results are available. A large number of measurements were made using the FM-CW systen with very similar results to those of the pulse system. 
Measurements of borehole returns were made at the Kaiser mine near Raton, New Mexico. The useable boreholes were located in a bench of coal approximately 1.6-m thick which was left in an open pit area of the mine. The borehole dianeter was $12 \mathrm{~cm}$. All the measurements made at the mine site were done with the FM-CW system.

The most notable result is that there was much more consistency from point to point in a series of measurements with the hole refilled and tamped with coal than in a similar series run with the boreholes either einpty or with a metal pipe inserted. Placing a pipe in the hole especially alters the response pattern over a wide frequency range and a wide spacial range much inore than would be indicated by simple theory. Probably, more theoretical work is needed on the FM-CW systeln before proceeding much further with hardware implementation.

Some series were run using a variable-density recorder to display results. It appears that this approach may be useful to help visually interpret a large arnount of data.

Long delays in delivery of equipment ordered for this project precluded additional tests that had been planned for that site and a location at the Pittsburgh seam.

\section{ADAPTATION OF SEISMIC DATA METHODS}

A local comnercial company, suggested by the Bureau of Mines, was approached to suggest methods for applying its proprietary seisinic data analysis techniques to electromagnetic pulse-type responses. The results of the initial discussion seem promising; but thus far in the program, we have not been able to provide the multiple results in the pattern which their method demands.

It should be noted that the suggested approach utilizes a modification of the common depth point (CDP) method. This is not an ideal approach for an underground radar, since one must know approximate range or position of the target in advance and multiple sets of measurements must be made. This technique is not anenable to a near, real-time scan method at a coal face, but nay have some value for other survey work. 


\section{FUTURE DIRECTIONS FOR RADAR DETECTION OF UNDERGROUND ANOMALIES}

\subsection{Mining Needs}

This contract was initiated with the goal of developing a system to locate old mine workings and old wells penetrating coal seams near present coal mining activity. The next phase in this ongoing program is to be devoted to the construction and testing of a model system that will detect these hazards. It is believed that the goals should be expanded to cover clay veins and faults in coal seams in any future effort. The time allocated to the overall effort would have to be increased, but the expenditures of funding would be more effective if applied to these closely related problems in the same program.

The location of clay veins and faults in coal seams has become of much greater importance in the last few years, since these situations are severe impediments to production, especially when mining is done with longwall machinery. The location of clay veins is important, since the coal seam may have different heights on each side. This situation is difficult to mine effectively with longwall machinery because the longwall machine cannot be angled up or down enough to accommodate the sudden shift in coal height. The clay can be hard ellough to damage the cutters on mining machines. The roof structure near faults or clay veins is usually unstable and requires extensive bolting. It would be helpful to know ahead that such a situation exists so as to start turning a longwall machine or to make the most economic choice on allocating manpower or machinery to the job of mining the clay vein. Since a clay vein may act as a partial barrier to methane drainage, the mining through a clay vein may suddenly release large quantities of gas. This gas can quickly becoine a safety problen which can be best solved when one is forewarned. As a consequence, one future goal should be to make any hazard detection systerl adaptable to use with longwall mining machines.

\subsection{Antennas}

The antennas developed for this work are useable with both the FM-CW system and pulse system. This development effort has been carried only as far as is consistent with progress in other areas of the work. The work done on them to this point indicates further improvenents in performance can be made. The present indications are that improvenents can best be brought about by careful use of dielectric loading to reduce the physical size, resistive loading to reduce unwanted reflections, and perhaps by investigating the general bow-tie antenna (see section 3) or a wideband microstrip approach [30]. 
This effort, in both the Phase I work as well as Phase II work, has been confined to exploiting the time domain or pulse-type system primarily and the Frequency-Modulated, Continuous-Wave (FM-CW) system to a lesser degree. Recent improvements to both systems continue to make them important candidates for an ultimate solution. Geophysical hazard detection is a very difficult problem. The solution requires many design compromises which subsequently limit the system applications and the analysis of the responses. At this writing, no technique is clearly superior to all others. It is important to this program that a number of methods are developed in parallel and that new designs are carefully evaluated.

NBS has done some introductory theory development work on the Planar Near Field Reconstruction (PNFR) technique and also, more recently, has made some beginning measurements to verify this theory [4]. The results have been good enough to suggest that this system should also be looked at very seriously in any underground radar mapping work.

\subsection{Accessibility to Mine Test Areas}

In any future effort, a test area meeting several requirements is crucial. It must have a well-described physical arrangement of rock and coal as well as the anomaly to be detected. The anomaly must be available at the proper ranges and be of the appropriate type. A very important feature of any test area is that access be available for a relatively long period of time. This should be done so that comparisons of test results can be made to detect small changes or inprovements in system performance as the work progresses.

The test range constructed by NBS during this phase has been invaluable to this work, but it does not provide all the elements needed. It is felt that a dedicated test area in an actual mine environment is necessary for this type of work.

\section{REFERENCES}

[1] Rumsey, V. H., Frequency Independent Antennas. Academic Press, New York $(1966)$.

[2] Carre1, R. L., The Characteristic Impedance of the Fin Antenna of Infinite Length. Antenna Laboratory Technical Report No. 16, Electrical Engineering Research Laboratory, Engineering Experiment Station, University of Illinois, Urbana, Illinois (1957).

[3] Lawton, R. A. and Ondrejka, A. R., Antennas and the Associated Time Domain Range for the Measurement of Impulsive Fields. Nat. Bur. Stand. (U.S.) Tech. Note $1008 ; 1978$ Novernber. 
[4] Jesch, R. L., Johnson, R. B., Belsher, D. R., Yaghjian, A. D., Steppe, M. C., and Fleming, R. W., High Resolution Sensing Techniques for Slope Stability Studies. FHWA-RD-79-32 (January 1979).

[5] Buettner, H. M., Antenna Design for Geophysical Application. URS I Digest, North American Radio Science Mtg., Laval University, Quebec, Canada (June 2-6, 1980).

[6] Lytle, R.J. and Laine, E. F., Design of a Miniature Directional Antenna for Geophysical Probing from Boreholes. IEEE Trans. on Geoscience Electronics, Vol. GE-16, No. 4, (Oct. 1978).

[7] Kanda, M., The Effects of Resistive Loading on TEM Horns. Nat. Bur. Stand. (U.S.) NBSIR 79-1601; 1979 August.

[8] Kanda, M., The Characteristics of a Linear Antenna with Tapered Resistive and Capacitive Loading. Digest of IEEE/AP-S International Symposium, Laval University, Quebec, Canada (June 2-6, 1980).

[9] Carrel, R. L., The Characteristic Impedance of an Infinite Biconical Antenna of Arbitrary Cross Section. Antenna Laboratory, Technical Report No. 25, Electrical Engineering Research Laboratory, Engineering Experiment Laboratory, Engineering Experiment Station, University of Illinois, Urbana, Illinois (1957).

[10] Schelkunoff, S. A., Electromagnetic Waves. D. Van Nostrand Co. Inc., New York, (1943).

[11] Schelkunoff, S. A., Advanced Antenna Theory. John Wiley and Sons, Inc., New York (1952).

[12] Kraus, J. D., Antennas. McGraw-Hi 17 Book Co, Inc., New York, (1950).

[13] Papas, C. H. and King, R., Input Impedance of Wide-Angle Conical Antennas Fed by a Coaxial Line. Proc. of I.R.E., pp. 1269-1271, (Nov. 1949)。

[14] Galejs, J., Antennas in an Inhomogeneous Medium. Pergamon Press, New York, (1969).

[15] Galejs, J., Dielectric Loading of Electric Dipole Antennas. Journal of Research of the Nat. Bur. Stand. (U.S.), Digest Radio Propagation, Vol. 660, No. 5, (Sept.-Oct. 1962).

[16] Balanis, C. A., Jeffrey, J. L., and Yoon, Y. K., Electrical Properties of Eastern Bituminous Coal as a Function of Frequency Polarization and Direction of the Electromagnetic Wave and Temperature of the Sample. IEEE Trans. on Geoscience Electronics, Vol. GE-16, No. 4 (Oct. 1978).

[17] Wheeler, H. A., Fundamental Limitations of Small Antennas. Proc. $\operatorname{IRE}(12), 1479-84,(1947)$. 
[18] Cook, J. C., Status of Ground-Probing Radar and Some Recent Experiments. Reprinted by Am. Soc. of Civil Engrs., from Proc. of Engineering Foundation Conference on "Subsurface Exploration for Underground Excavation and Heavy Construction", Henniker, N.H., Aug. 1974.

[19] Cook, J. C., Radar Transparencies of Mine Tunnel Rocks. Geophysics, vol. 40, pp. $865-885,1975$.

[20] Berkowitz, R. S., Modern Radar (Analysis, Evaluation, System Design). John Wiley \& Sons, New York, 1965.

[21] Bussey, H. E.; Larsen, E. B., Buried Antenna Performance; Development of Small Resonant Antennas. Rome Air Development Center, Griffiss AFB, New York, RADC-TR-74-169, June, 1974 (Defense Documentation Center No. AD 783-274).

[22] Hufford, G. A., UHF Propagation from Buried Antennas. ESSA Research Laboratories, ITS, Tech. Memorandum ERLTM-ITS191, 68 pp, July, 1969.

[23] Mumford, W. W., Scheibe, E. H., Noise Performance Factors in Communication Systenis. Horizon House-Microwave, Inc., 1968.

[24] Bowman, J. J., Senior T. B. A., and Uslenghi, P. L. E., Electromagnetic and Acoustic Scattering by Simple Shapes. North Holland Pub. Co., Amsterdan, 1969.

[25] Kerr, D. E., Propagation of Short Radio Waves. McGraw Hill Book Co., New York, 1951

[26] Ruck, G. T., Barrick, D. E., Stuart, W. D., and Krichbaum, C. K., Radar Cross Section Handbook. Plenum Press, New York, 1970.

[27] Tarantolo, P. J. and Unterberger, R. R., Radar Detection of Boreholes in Advance of Mining. Geophysical Prospecting, vol. 26, pp. 359-382, 1978.

[28] Wait, J. R., Electromagnetic Radiation from Cylindrical Structures. Pergamon Press, New York, 1959.

[29] Jesch, R. L., Void Detection. Final report to Navy, National Bureau of Standards, Boulder, CO (August, 1980).

[30] I-Ping, Yu, Multiband Microstrip Antenna. NASA Tech Briefs, vol. 5, no. 1, Spring 1980.

[31] Jenkins, F. A.; White, H. E., Fundamentals of Physical Optics. McGrawHill, 1937.

[32] Brysk, H., The radar cross section of a semi-infinite body. Can. Jour. Physics, vol. 38, pp. 48-56, 1960. 
[33] Bussey, H. E.; Richmond, J. H., Scattering by a lossy dielectric circular cylindrical multilayer, numerical values. IEEE Trans. on Ant. \& Propa., vol. AP-23, pp. 723-725; September 1975.

[34] Baños, A., Jr., Dipole radiation in the presence of a conducting halfspace. Pergamon Press, New York, 1966.

[35] Brekhovskikh, L. M., Waves in layered media. Academic Press, New York, Second Edition, 1980.

[36] Stratton, J. A., Electromagnetic theory. McGraw-Hill, New York, 1941.

[37] Engheta, N.; Papas, C.H.; Elachi, C., Radiation patterns of interfacial dipole antennas. Radio Science, vol. 17, No. 6; November-December 1982.

[38] Lewis, R. L., Far-field computaion inside a dielectric media for an antenna above a planar interface. National Radio Science Meeting, U.S. Nat. Committee/URS I, 24-28 May 1982, Univ. of New Mexico, Albuquerque.

[39] Martin, L. C.; Welford, W. T., Technical optics, vol. I. Pitman Pub. Corp., New York, 1966 (second edition).

[40] Wittmann, R. C., Simulated pulse techniques for measuring coal layer thickness. Ch. 6, pp. 287-297 in J. R. Wait, Ed., Analytical bases for electromagnetic sensing of coal properties, final technical report to U.S. Dept. of Energy ET-75-G-01-8972, JuTy 1978.

[41] Roe, K. C.; Wittmann, R. C., Improved coal interface detector, report to U.S. Dept. of Energy. Nat. Bur. Stand. (U.S.) NBSIR-82-1663, 1982 May.

[42] Lynch, A. C., Relationship between permittivity and loss tangent. Proc. IEEE (London), vol. 188, pp. 244-246, 1971. 


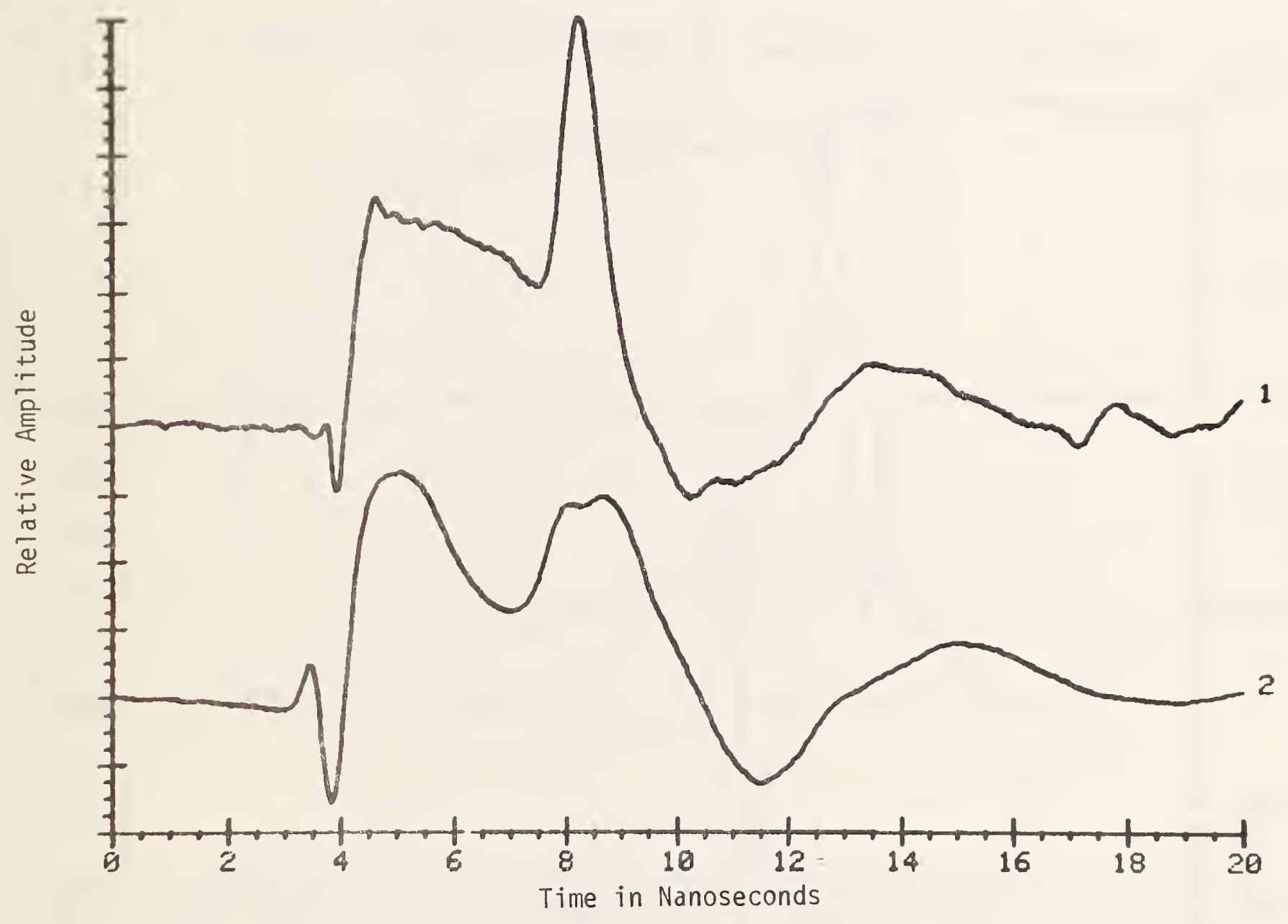

Figure 1. Reflections due to the NBS-TQ antenna without the resistive material (1) and the same antenna with the resistive material (2). 


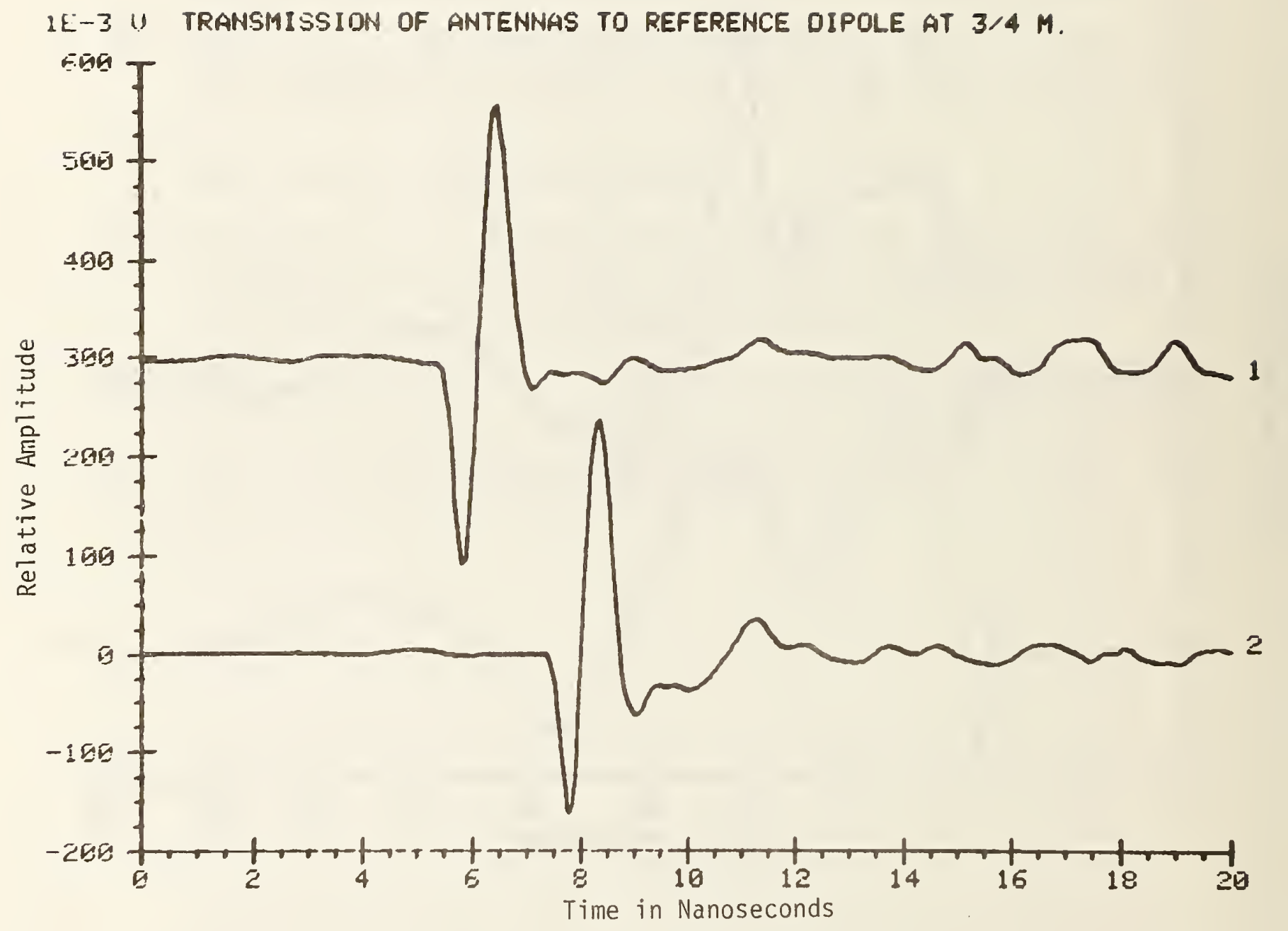

Figure 2. Transmitted waveform from the TG antenna (curve 1) and the TQ antenna (curve 2). 
$\mathrm{dB}$ above $1 \mathrm{~V} / \mathrm{M} / \mathrm{V}$

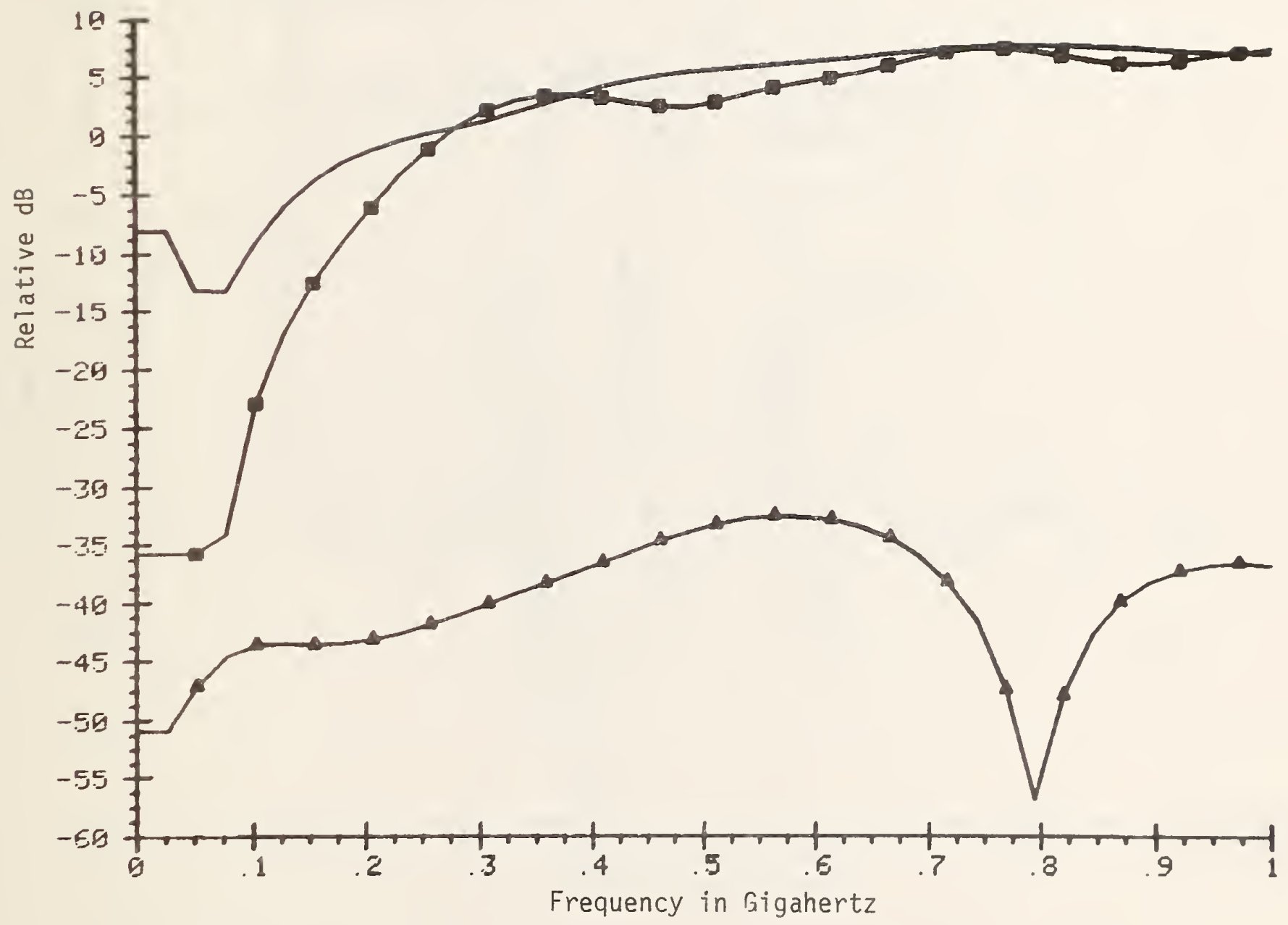

Figure 3. Relative transmission in $d B$ for the $T G$ antenna ( $p l a i n$ line) forward direction, the TQ antenna (squares) forward direction and the TQ antenna (triangles) reverse direction at a range of $3 / 4 \mathrm{~m}$. 


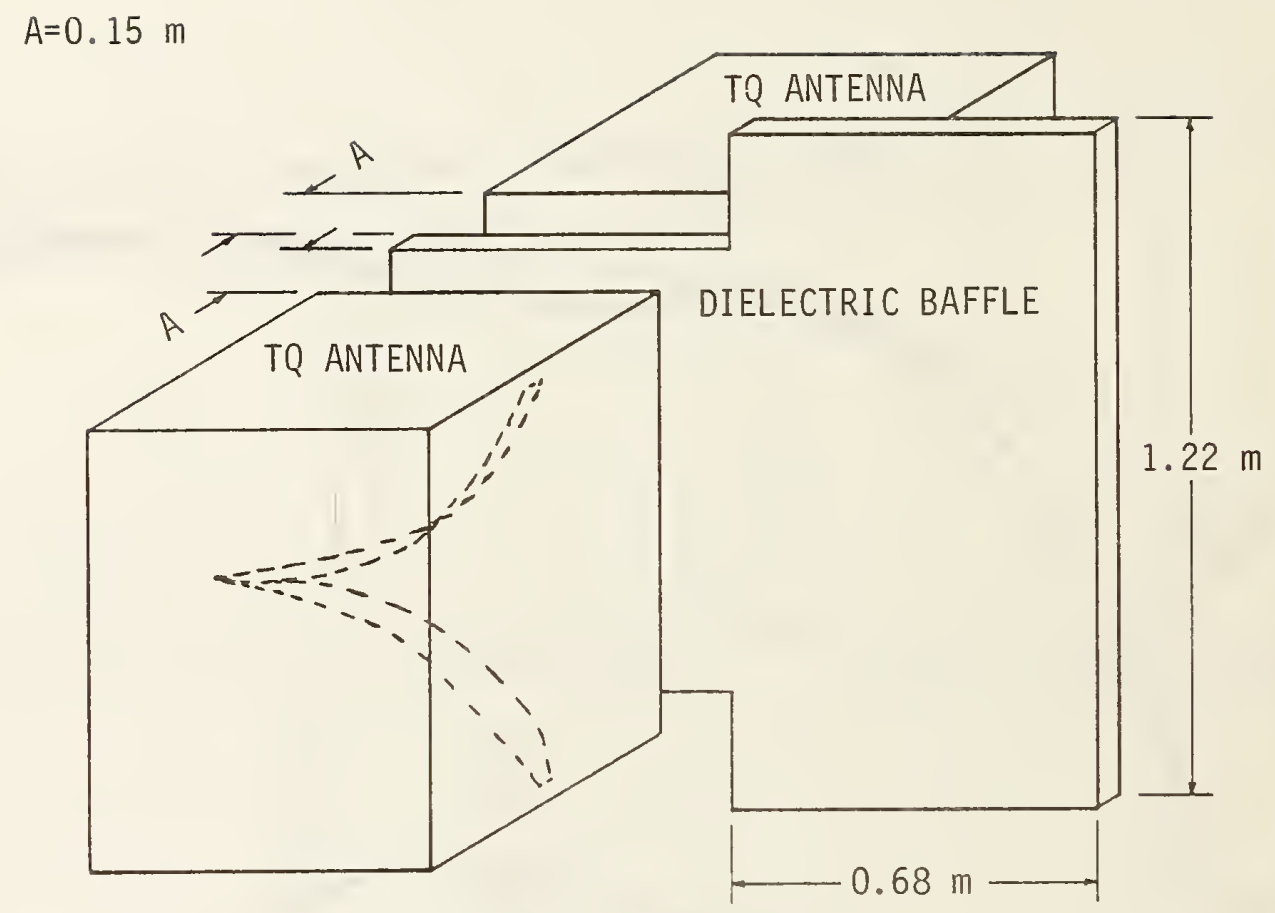

Figure 4. Baffle arrangement for isolating TQ antennas when operated bistatically. 


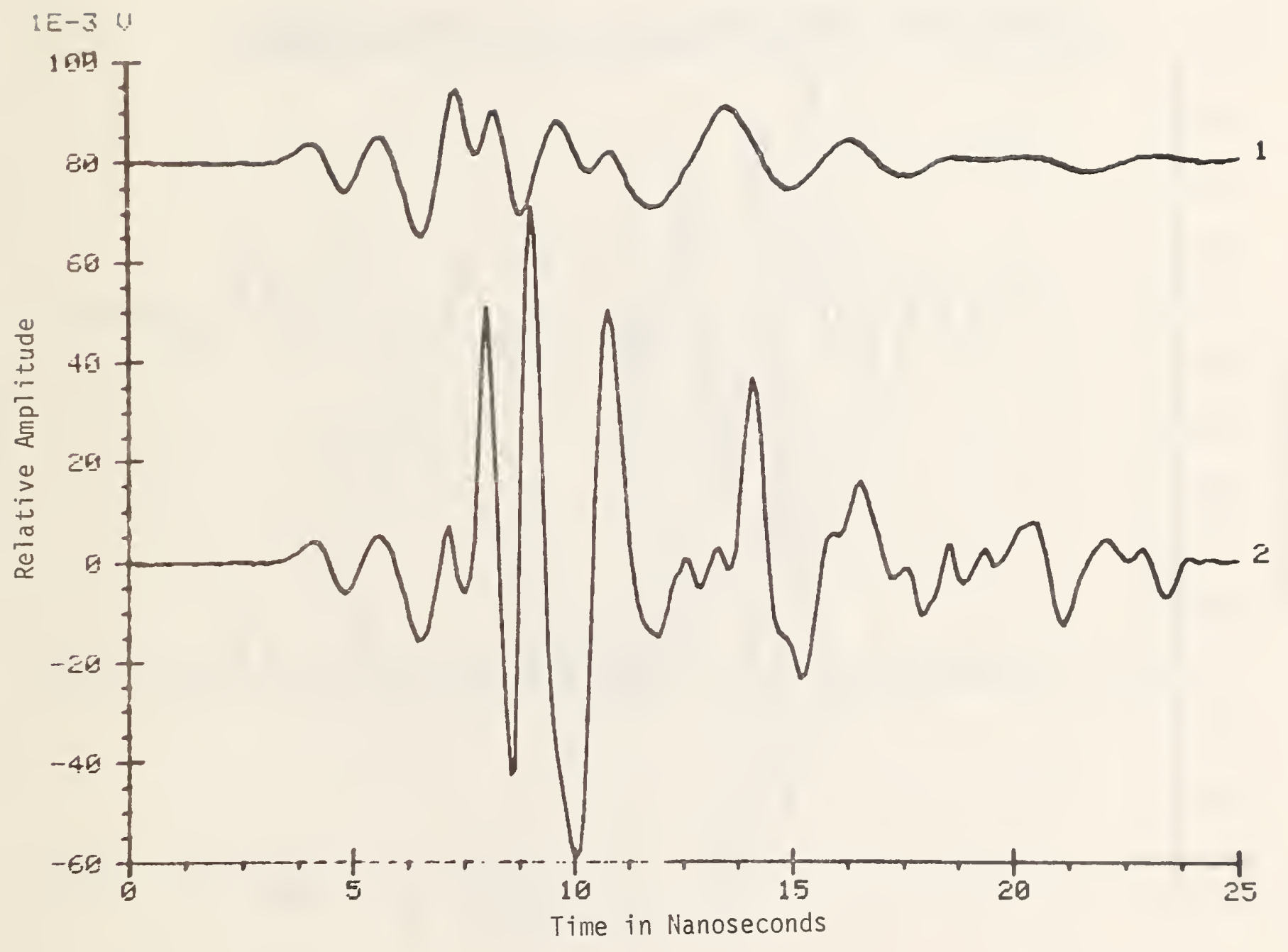

Figure 5. Response (1) of the system with the TQ antenna pair pointed at the sky and (2) at a 1.2 meter square metal plate 5 an in front of the baffle. 


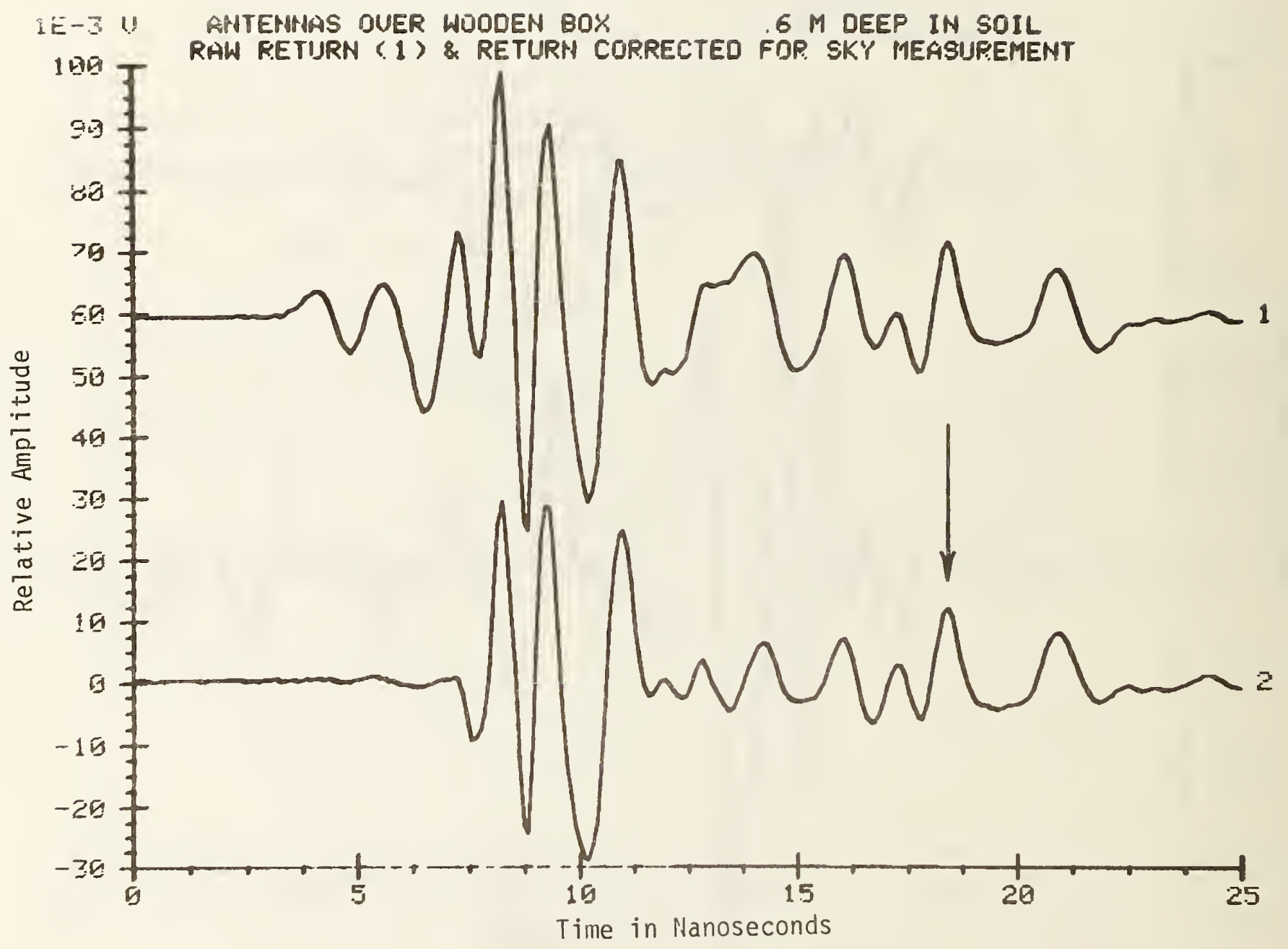

Figure 6. Pulse system antenna return from an air filled box target buried at 0.6 meter (1) and the same return corrected by subtraction of the sky return (2). 


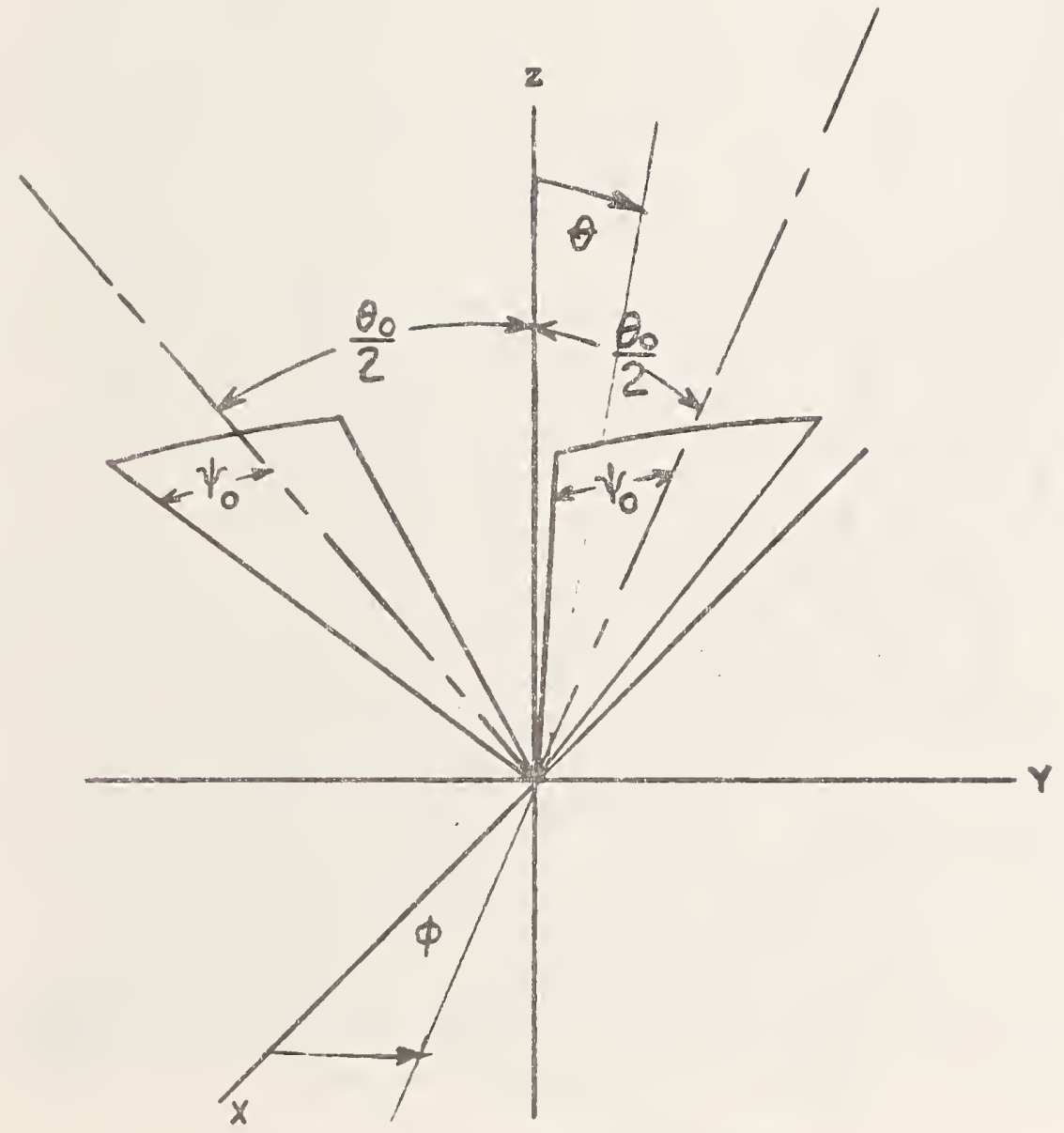

Fiqure 7. Noncoplanar fin antenna schematic. 


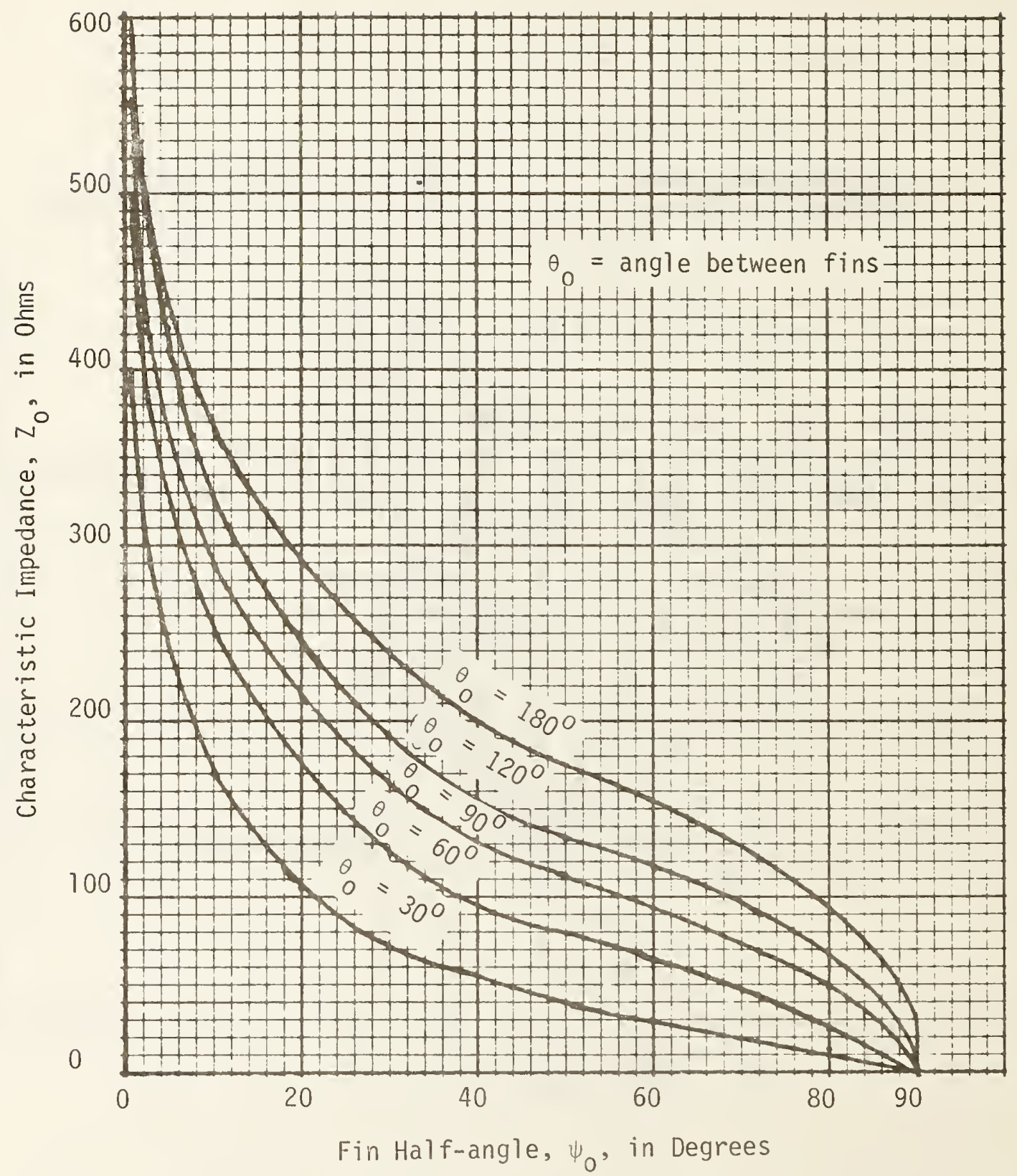

Figure 8. Characteristic impedance of the non-coplanar fin antenna with equal fin angles (plotted from data presented by Carrel [9]). 


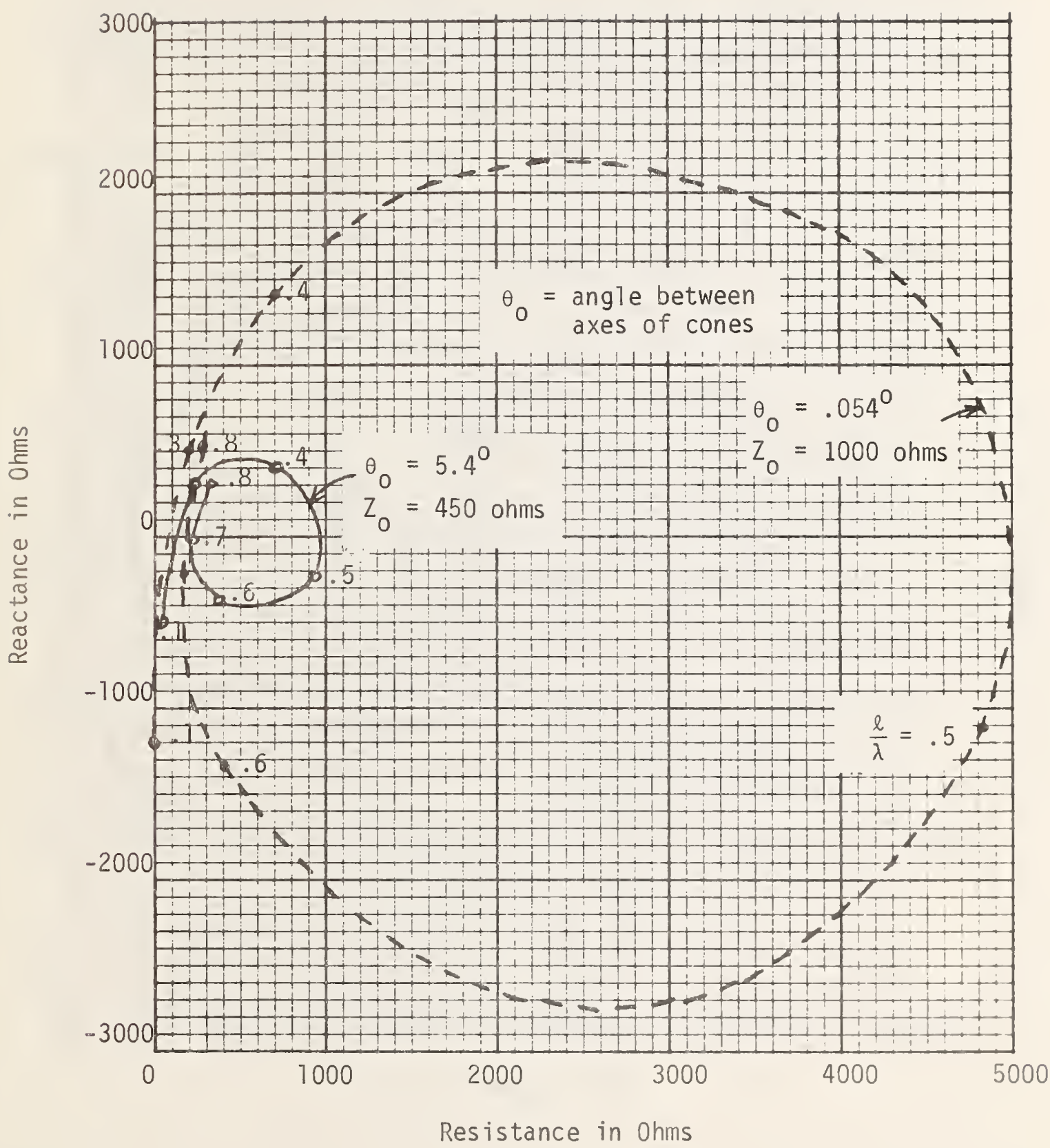

Figure 9. Input impedance of biconical antennas with $5.4^{\circ}$ cone angle and $.054^{\circ}$ cone angle as a function of the length, $\ell$, of the cone in wavelengths, $\lambda$ (plotted from data presented by Kraus [12]). 


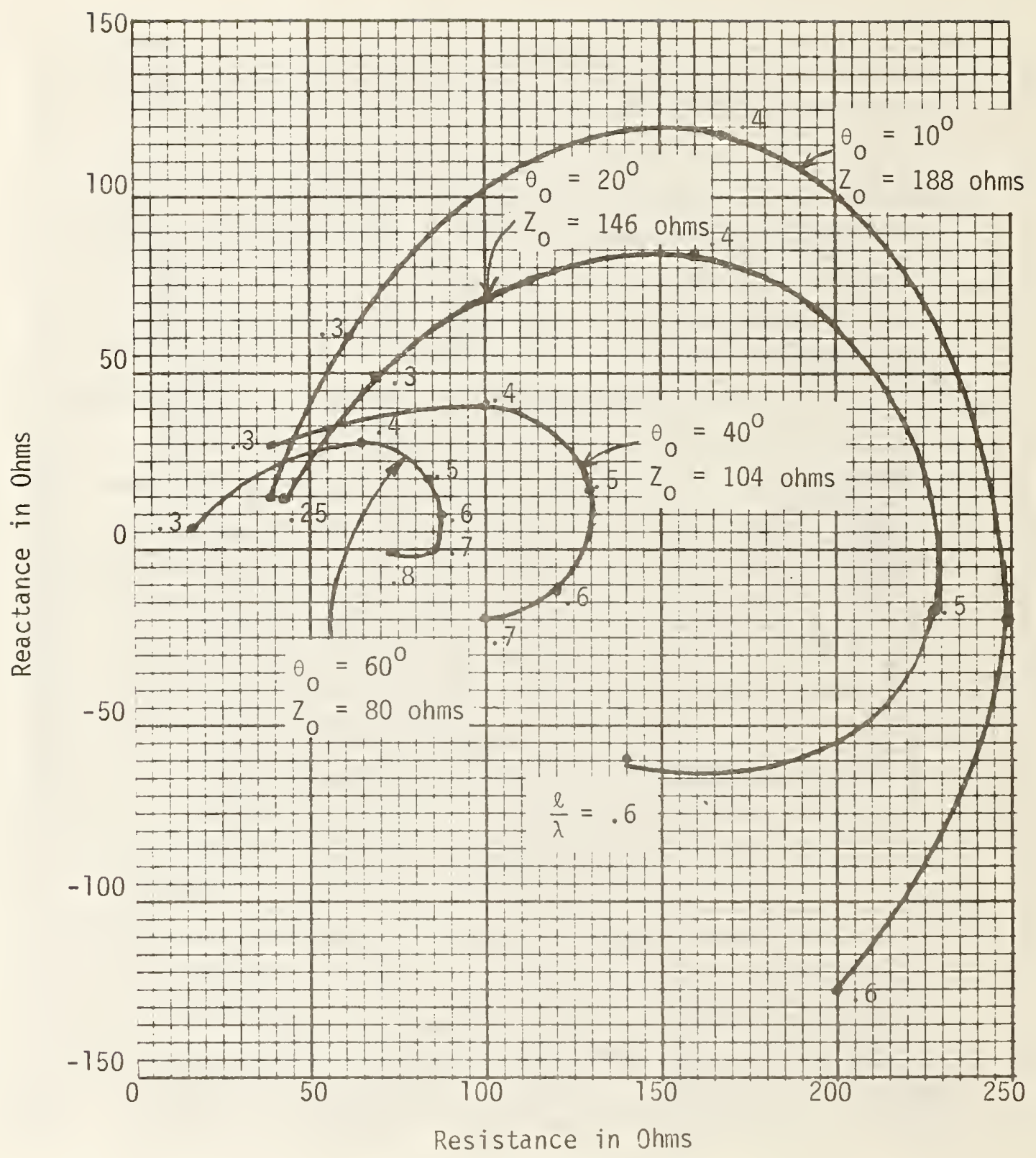

Figure 10. Input impedance of single cones with ground plane as a function of cone length, $\ell$, in wavelengths, $\lambda$. Cone angles are $10^{\circ}, 20^{\circ}$, $40^{\circ}$ and $60^{\circ}$ (plotted from data presented by Kraus [12]). 


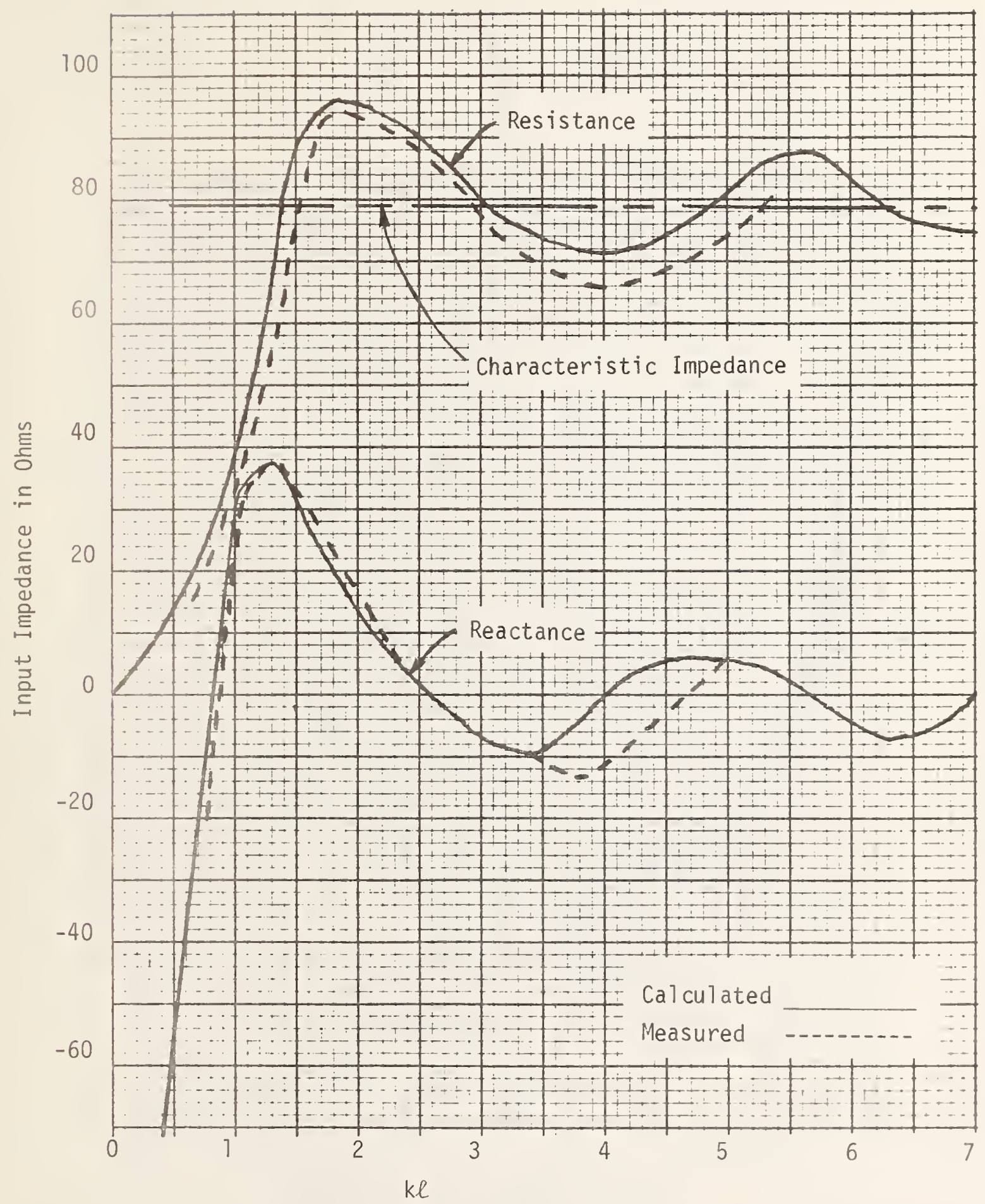

Figure 11. Input impedance of single cone with ground $\mathrm{pl}$ ane versus $k \ell . K$ is the wave number, $\ell$ is the cone length and the cone angle, $\theta_{0}$, is $60^{\circ}$ (plotted from data presented by Papas and King [13]). 


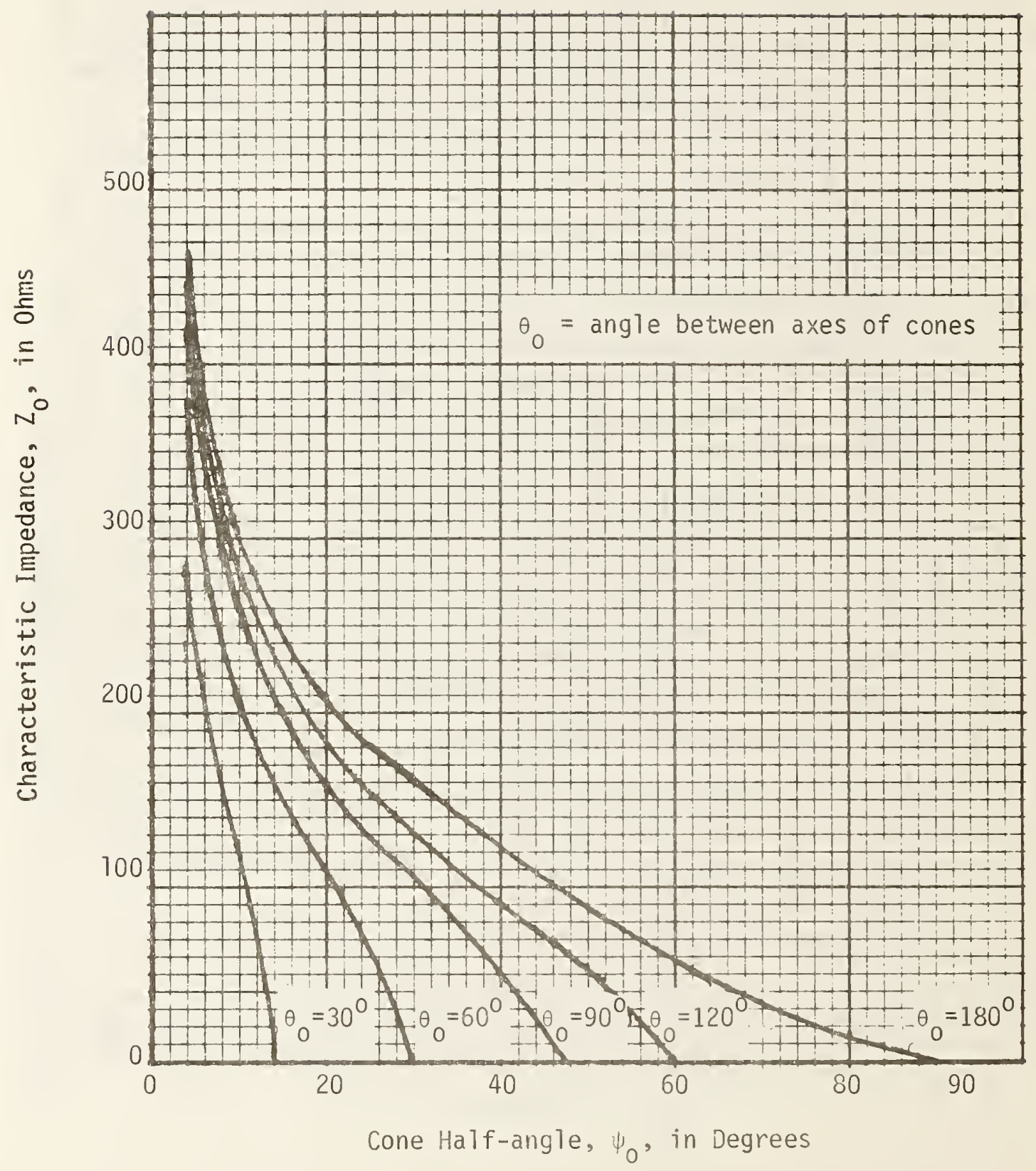

Figure 12. Characteristic impedance of a biconical antenna with equal cone angles, $\Psi_{0}$ (plotted from data presented by Carrel [9]). 


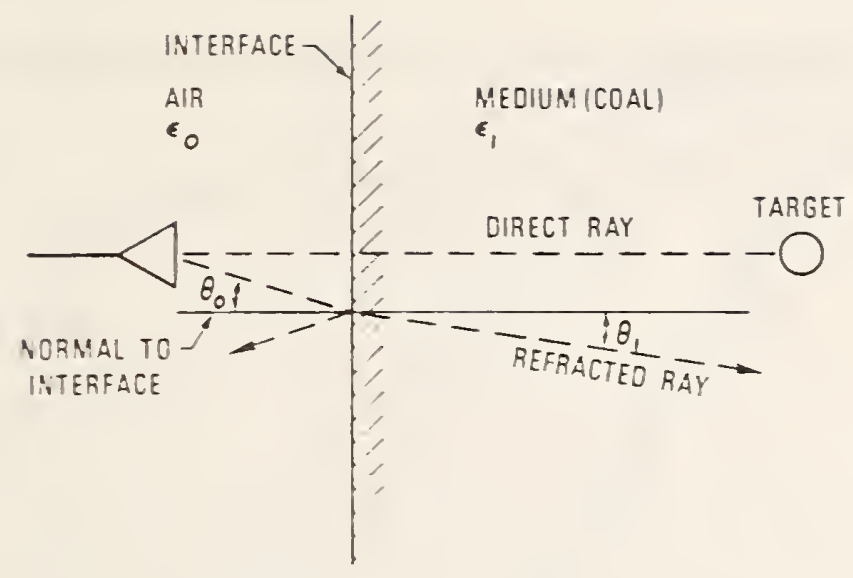

Finure 13a. Sketch of rays from source to field point (target). Rays are refracted into the second medium and partially reflected, $n_{0}<n_{1}$.

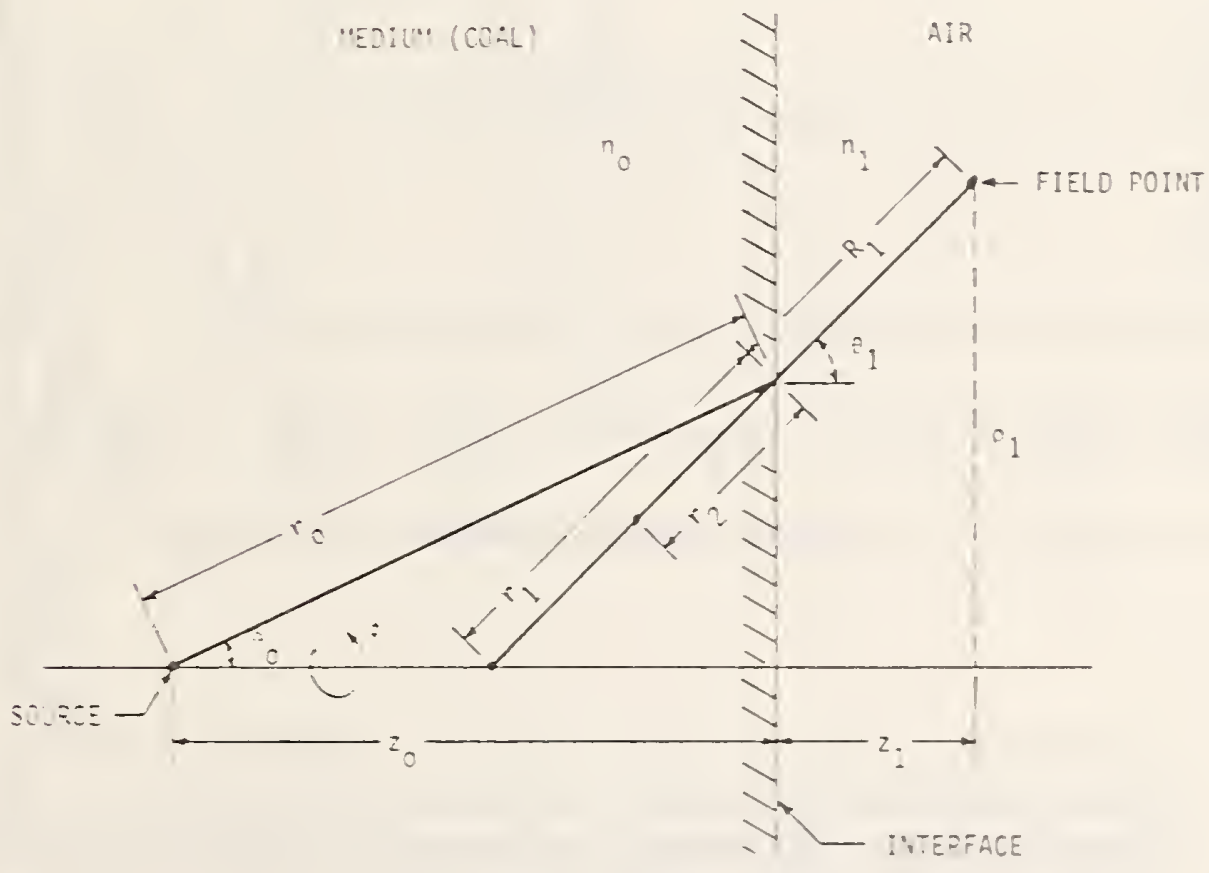

Figure:30. Geometry for calcuiating Sommerfeld interface transmission. in inis example, the source is in the denser medium with refractive index $n_{0}>n_{1}$. 


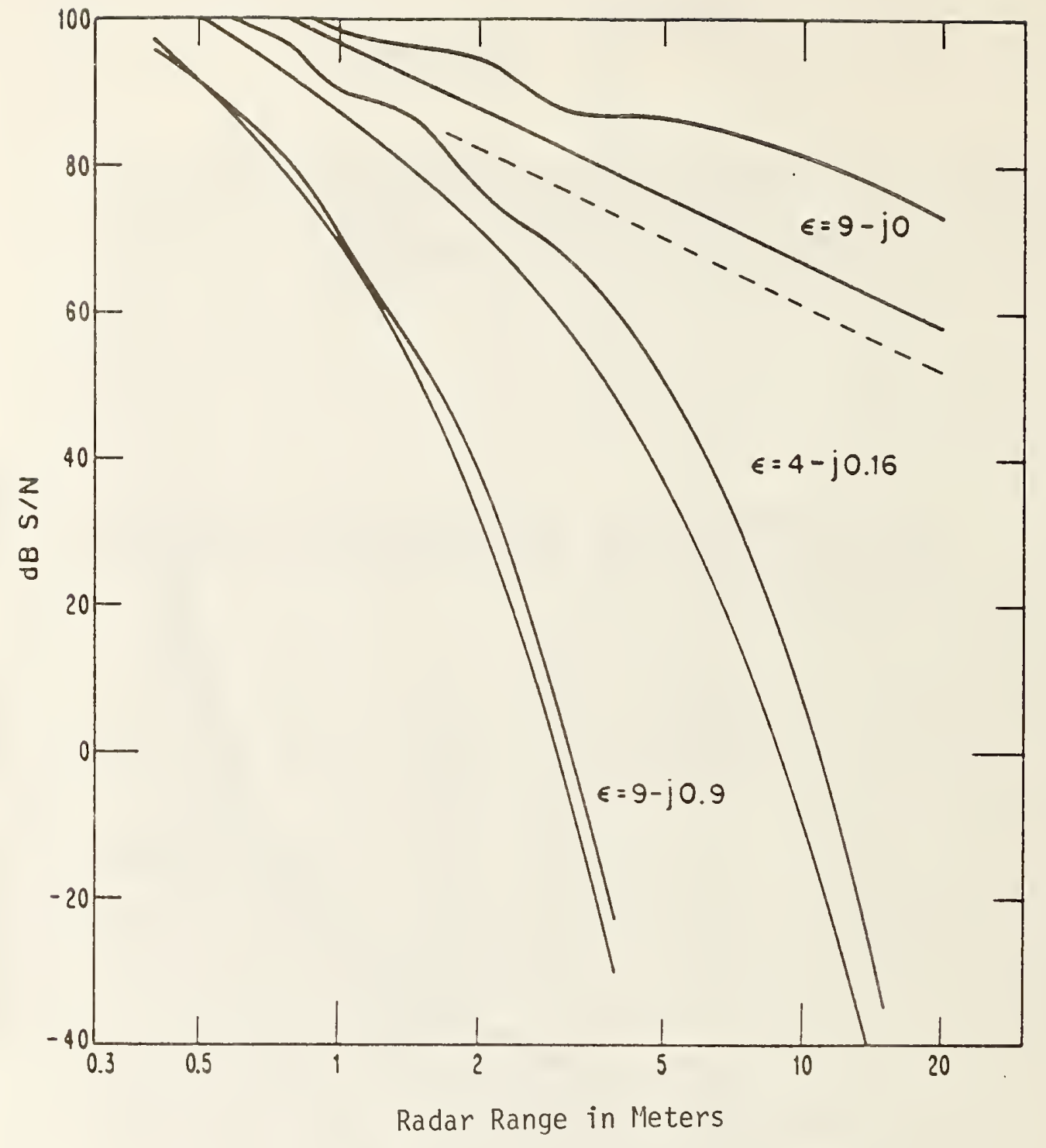

Figure 14. Signal-to-noise ratio for a $\mathrm{Cl}$ radar system with the following parameters: $500 \mathrm{MHz}$ source; horn antennas 50.8 an by $76.2 \mathrm{~cm}$; frequency $=0.5 \mathrm{GHz}$; complex permittivity $=9$-j0 for low loss curves, 4-j0.16 for medium loss curves, 9-j0.9 for high loss curves. The upper curve of each group is for a flat mine tunnel wall 1.5 meters high of infinite length with water in the tunnel. The lower sol id curve of each group is for a $30 \mathrm{~cm}$ diameter cased borehole. The dashed curve is for a water filled borehole without casing having a $30 \mathrm{~cm}$ diameter. The system noise is assumed to be $8 \mathrm{~dB}$ greater than Johnson noise in a $1 \mathrm{MHz}$ bandwidth and clutter is ignored. 


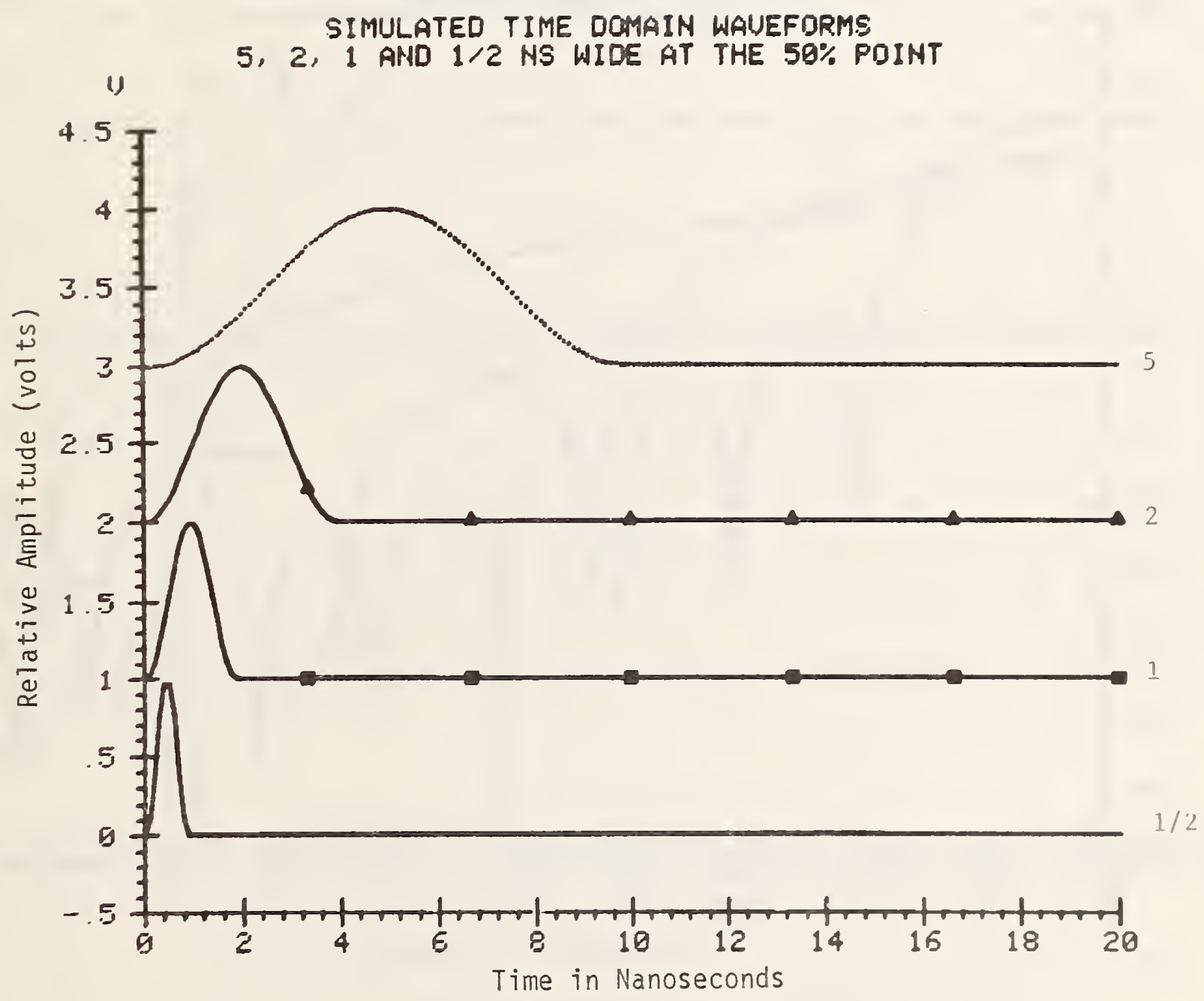

Figure 15. Simulated impulse waveforms having widths of $5,2,1$, and $1 / 2$ ns at the $50 \%$ point. 


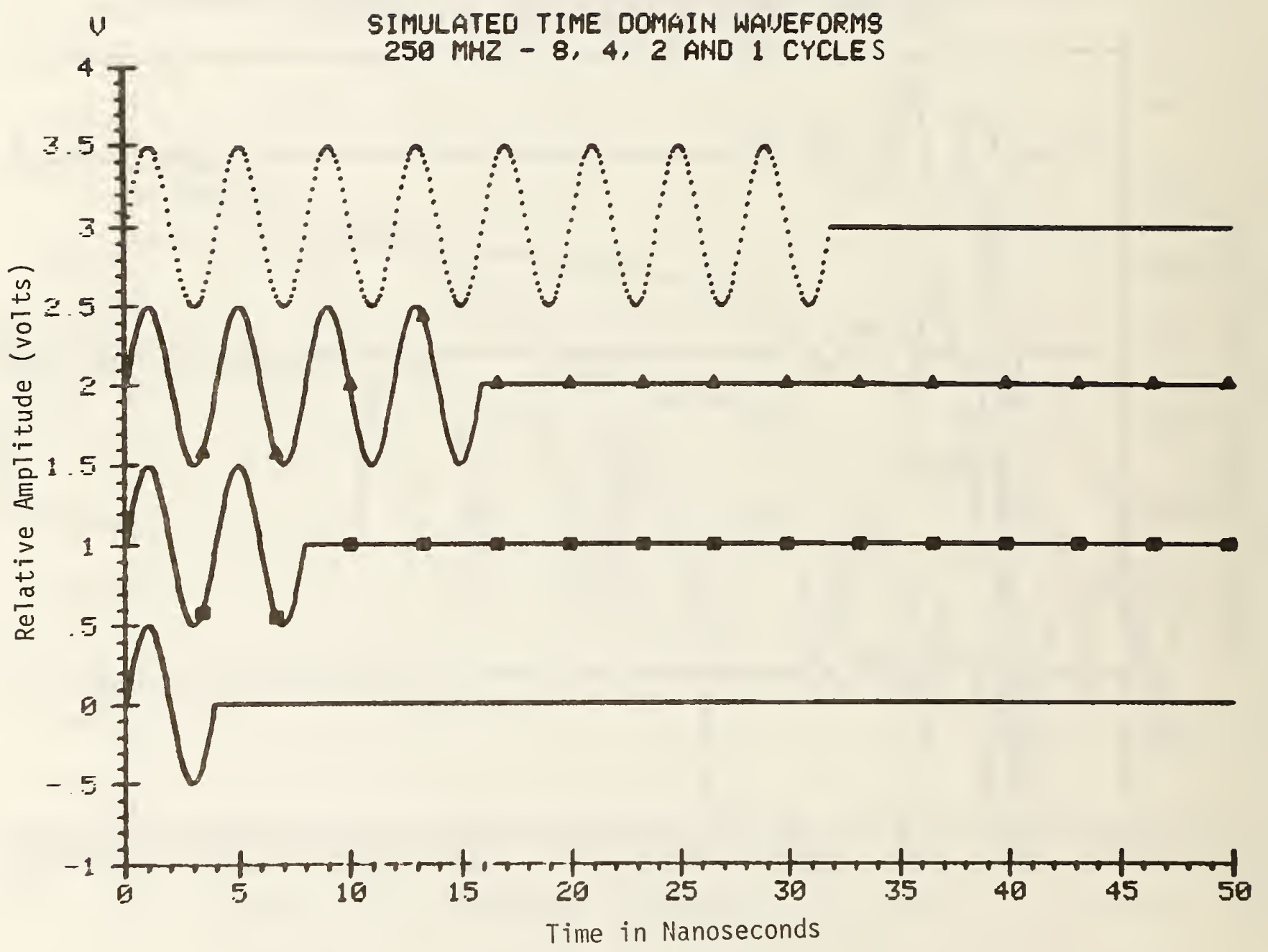

Figure 18. Simulated sinusoidal pul se waveforms having $8,4,2$, and 1 cycles. 


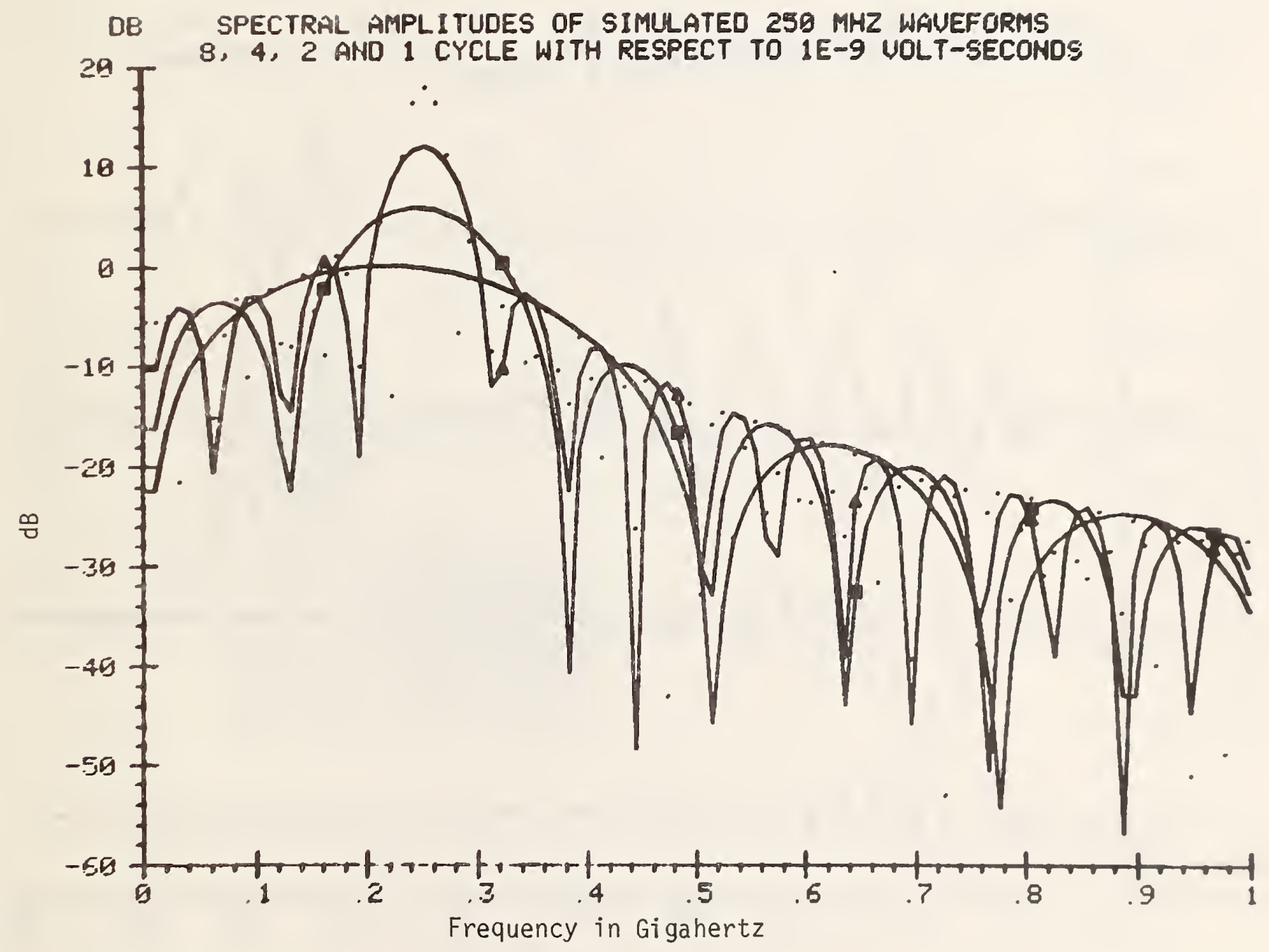

Figure 19. Resultant spectral amplitude of the sinusoidal pulses shown in figure 18. 8-cycle pulse, dotted curve; 4-cycle pulse, triangles; 2 -cycle pulse, squares; 1 -cycle pulse, sol id line with no marker. 


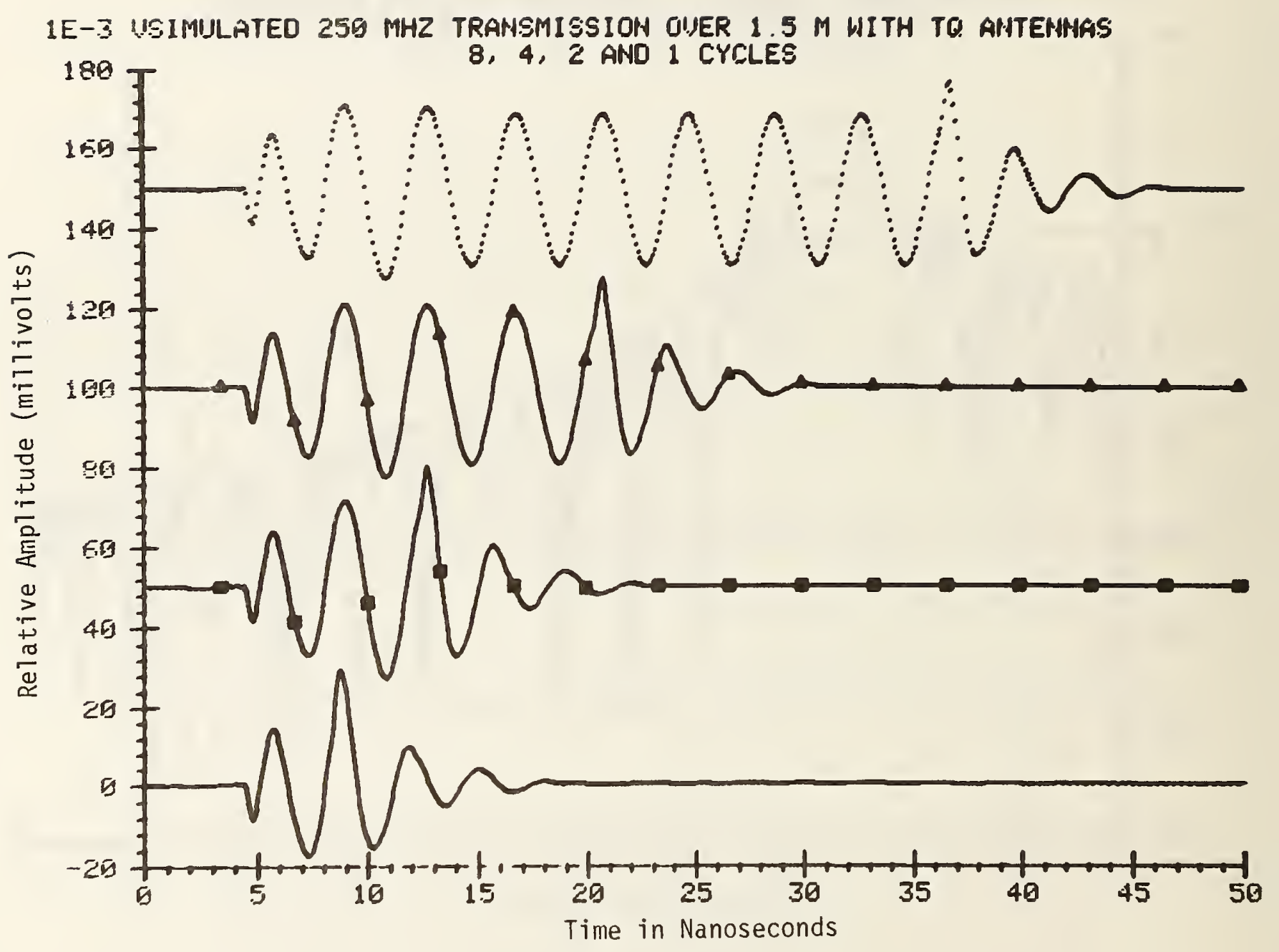

Figure 20. The signals resulting from the transmission of the sinusoidal pul se waveforms shown in figure 18 over a 1.5 meter path using TQ antennas. 


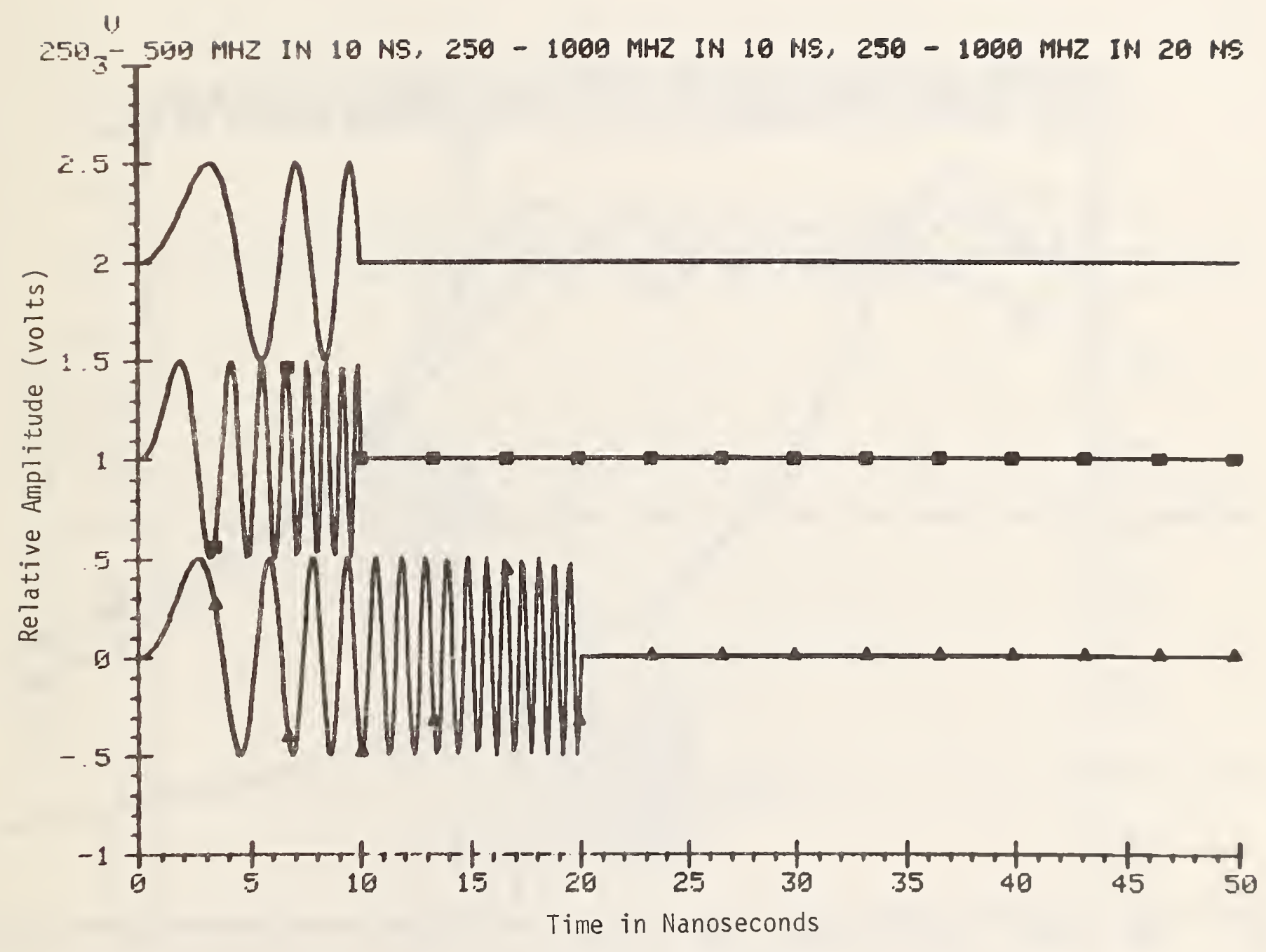

Figure 21. Simulated linear chirp pul se waveforms.

250-500 $\mathrm{MHz}$ in 10 nanoseconds, plain curve.

250-1000 MHz in 10 nanoseconds, square markers.

250-1000 MHz in 20 nanoseconds, triangle markers. 


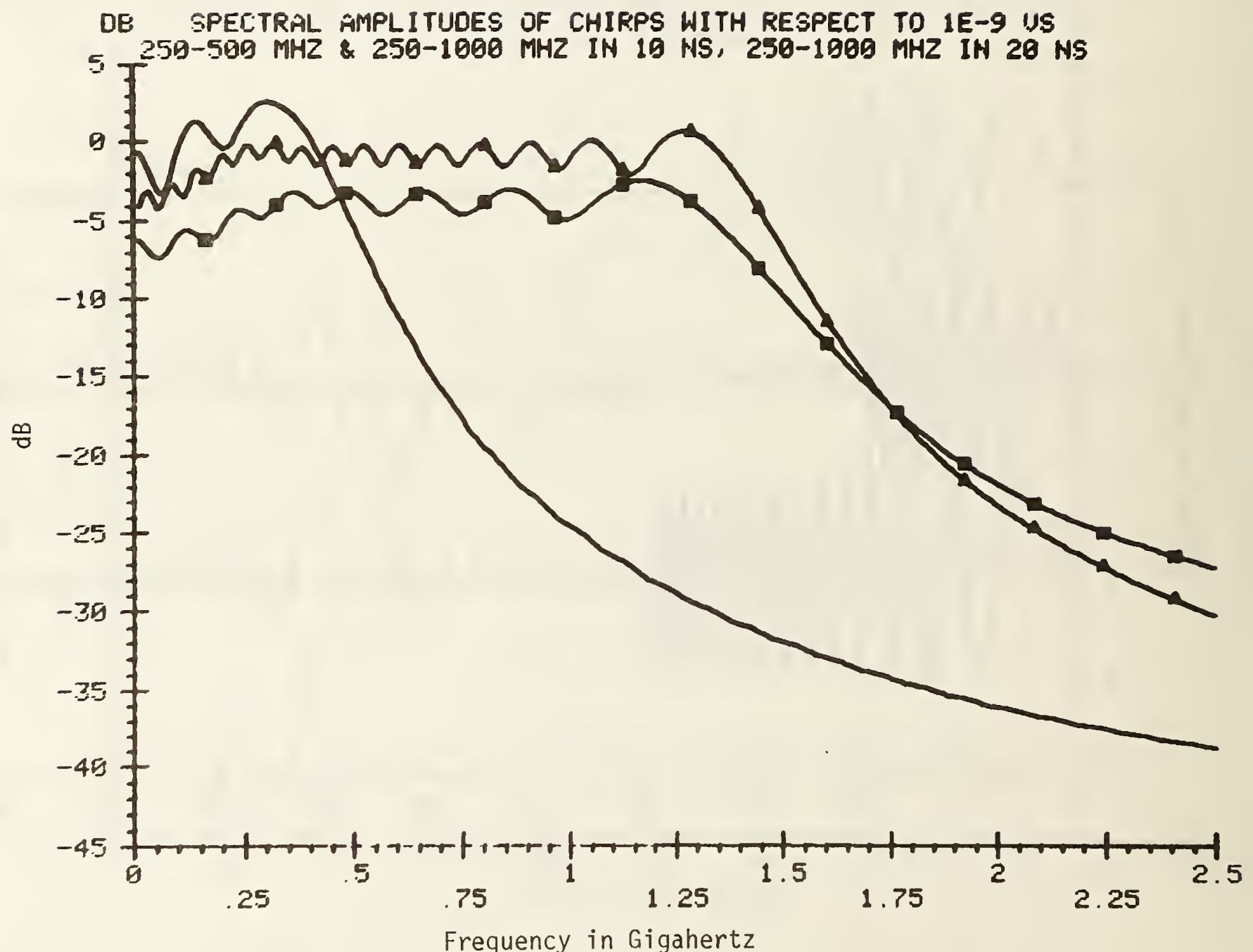

Figure 22. Resulting spectral amplitudes of the linear chirp pulse waveforms shown in figure 21. The curve markers correspond to figure 21. 


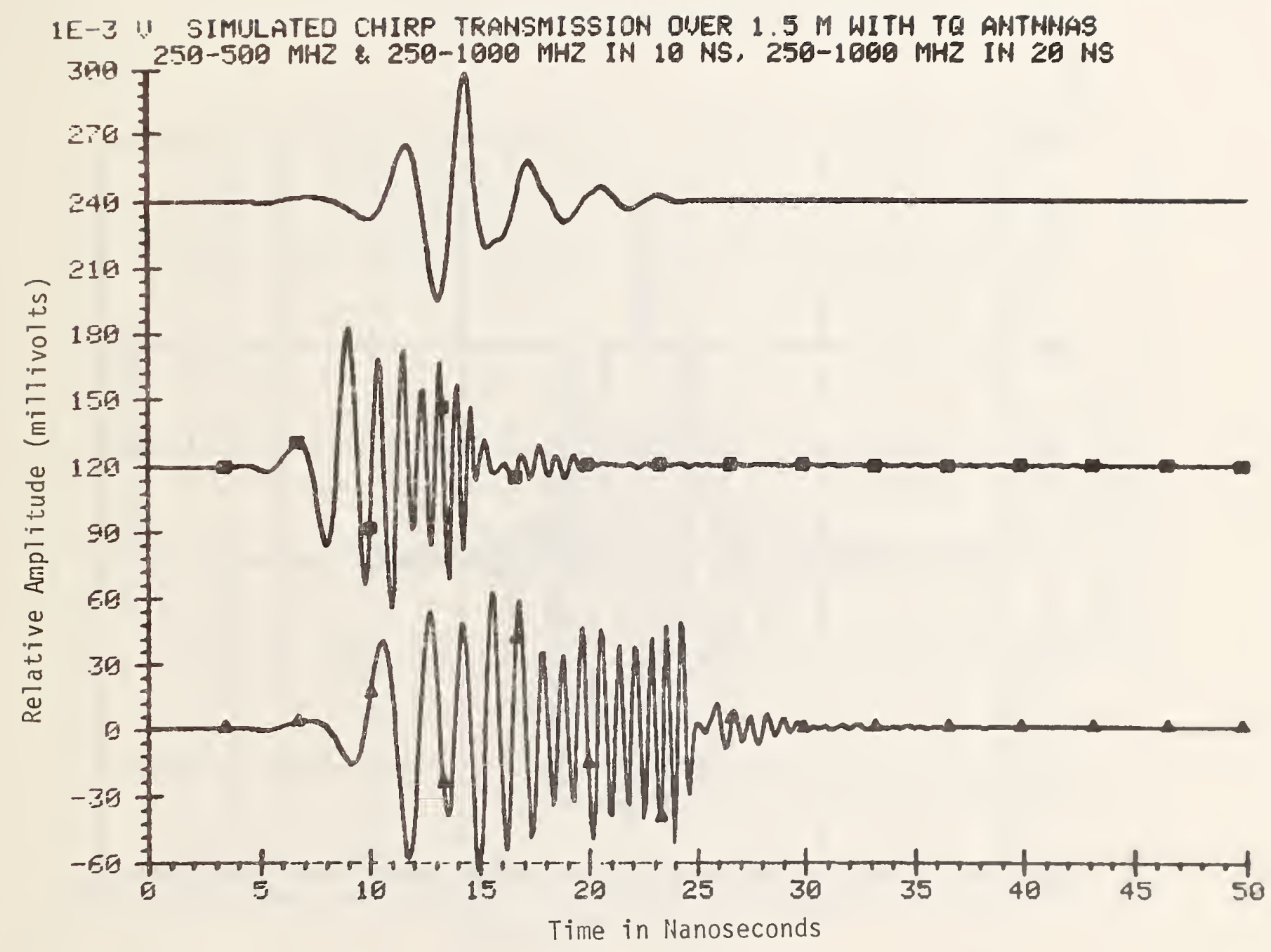

Figure 23. Resulting signals after transmission of the linear chirp pulses over a 1.5 meter path using TQ antennas. The curve markers correspond to figure 21. 


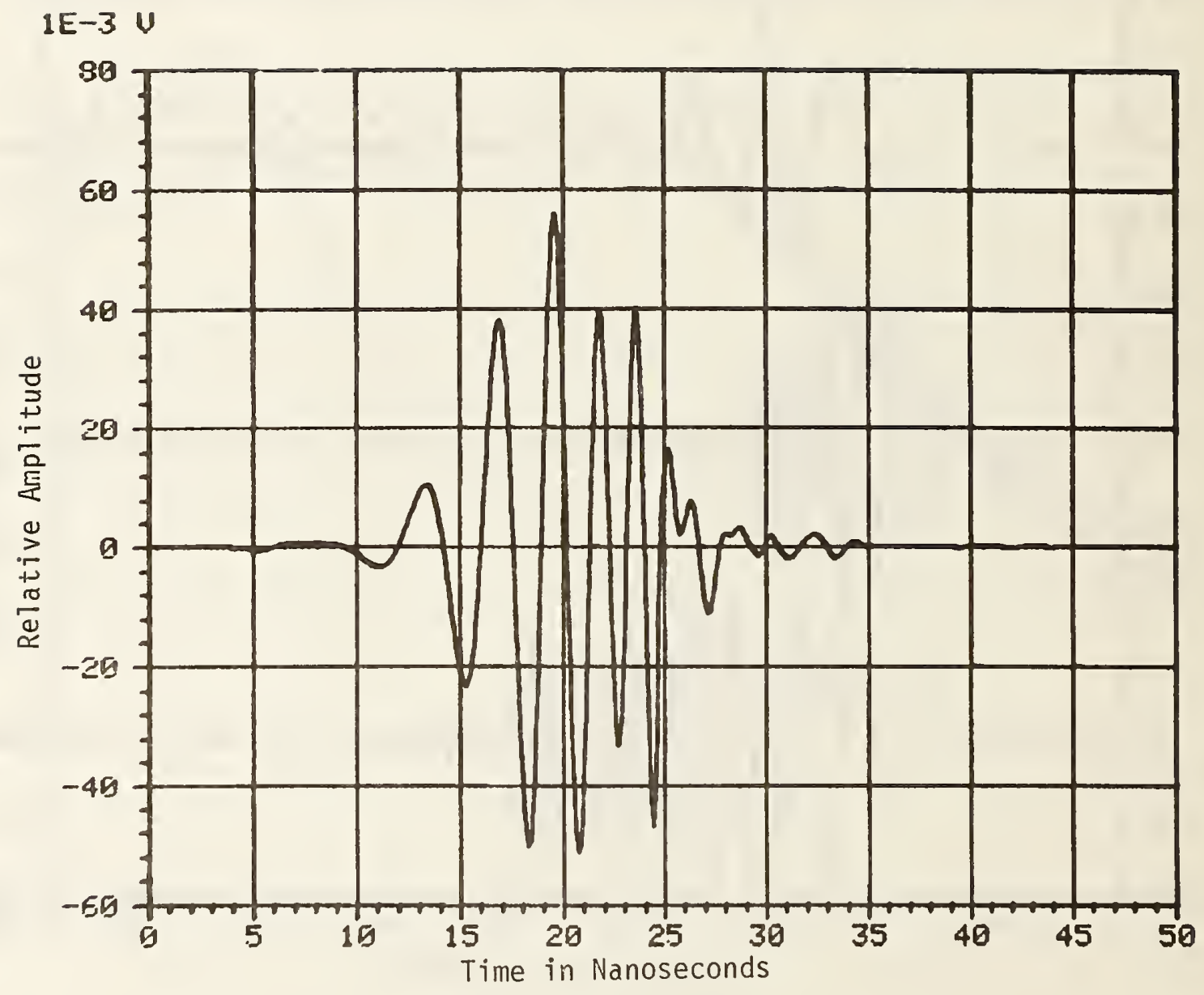

Figure 24. The result of transinitting a 300-600 MHz linear chirp pulse over a 1.5 meter path using the TQ antennas. 


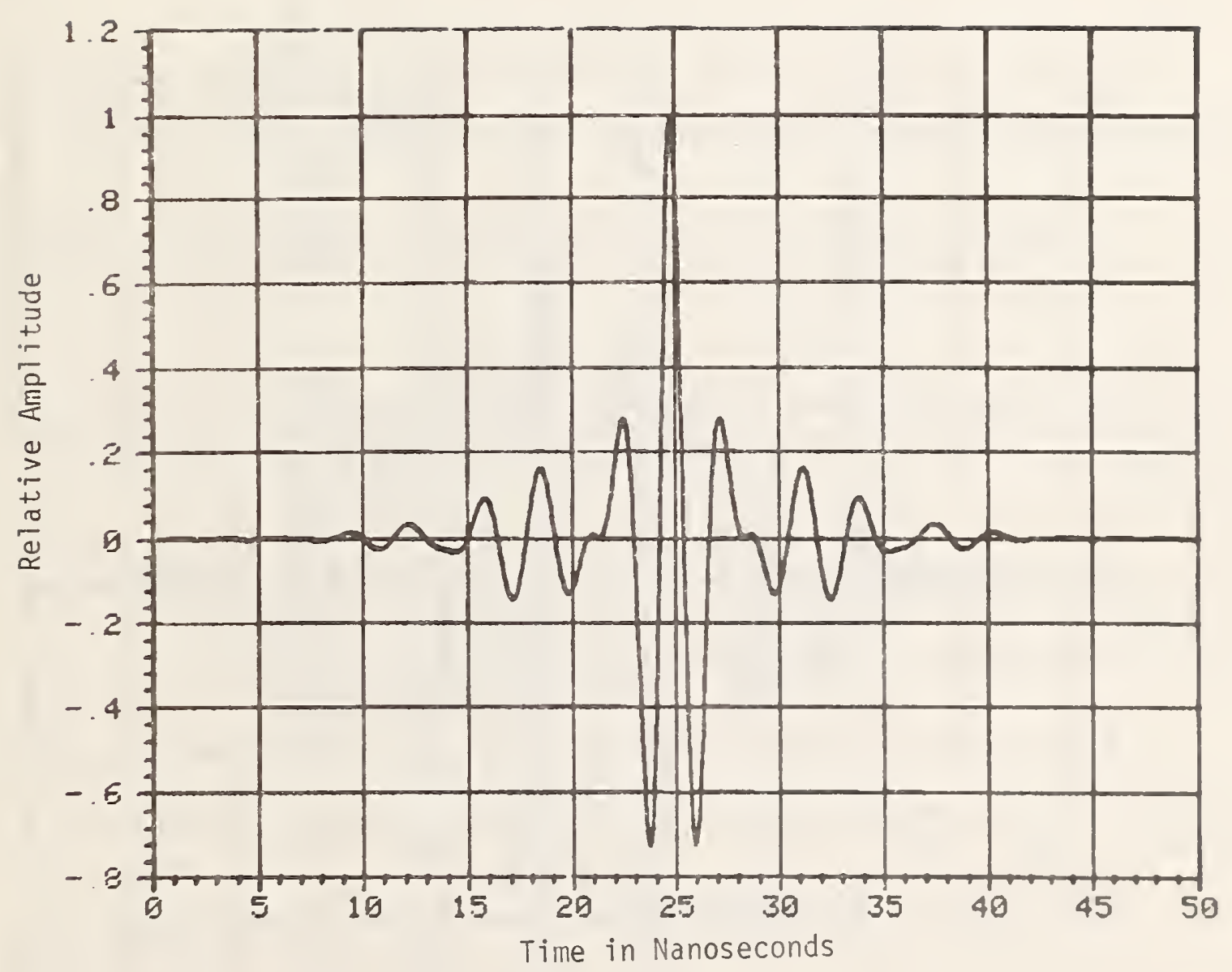

Figure 25. The result of autocorrelation of the waveform of figure 24 . 


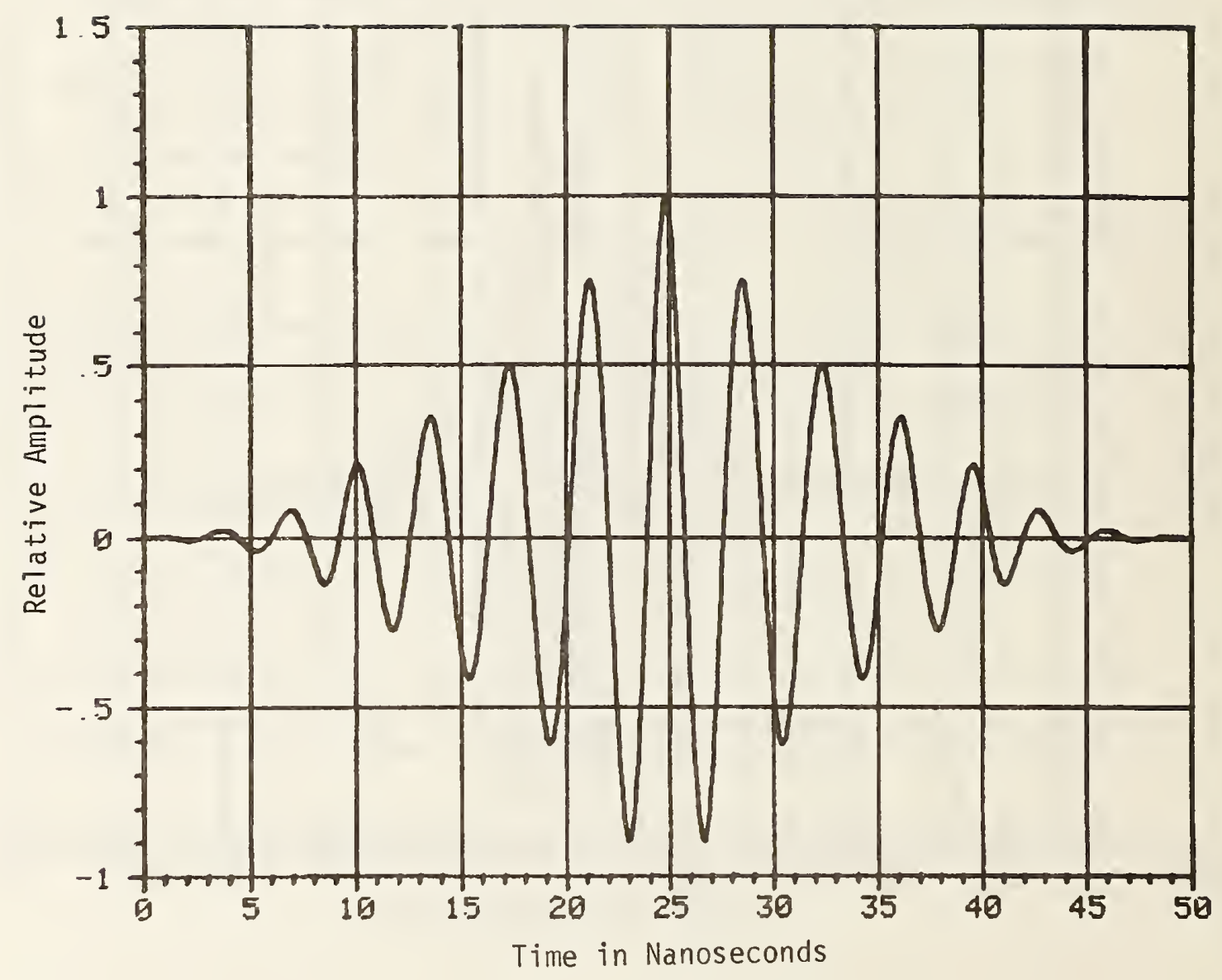

Figure 26. Autocorrelation of the transmitted waveform of the 4 cycle, 250 $\mathrm{MHz}$ sinusoidal pulse. 


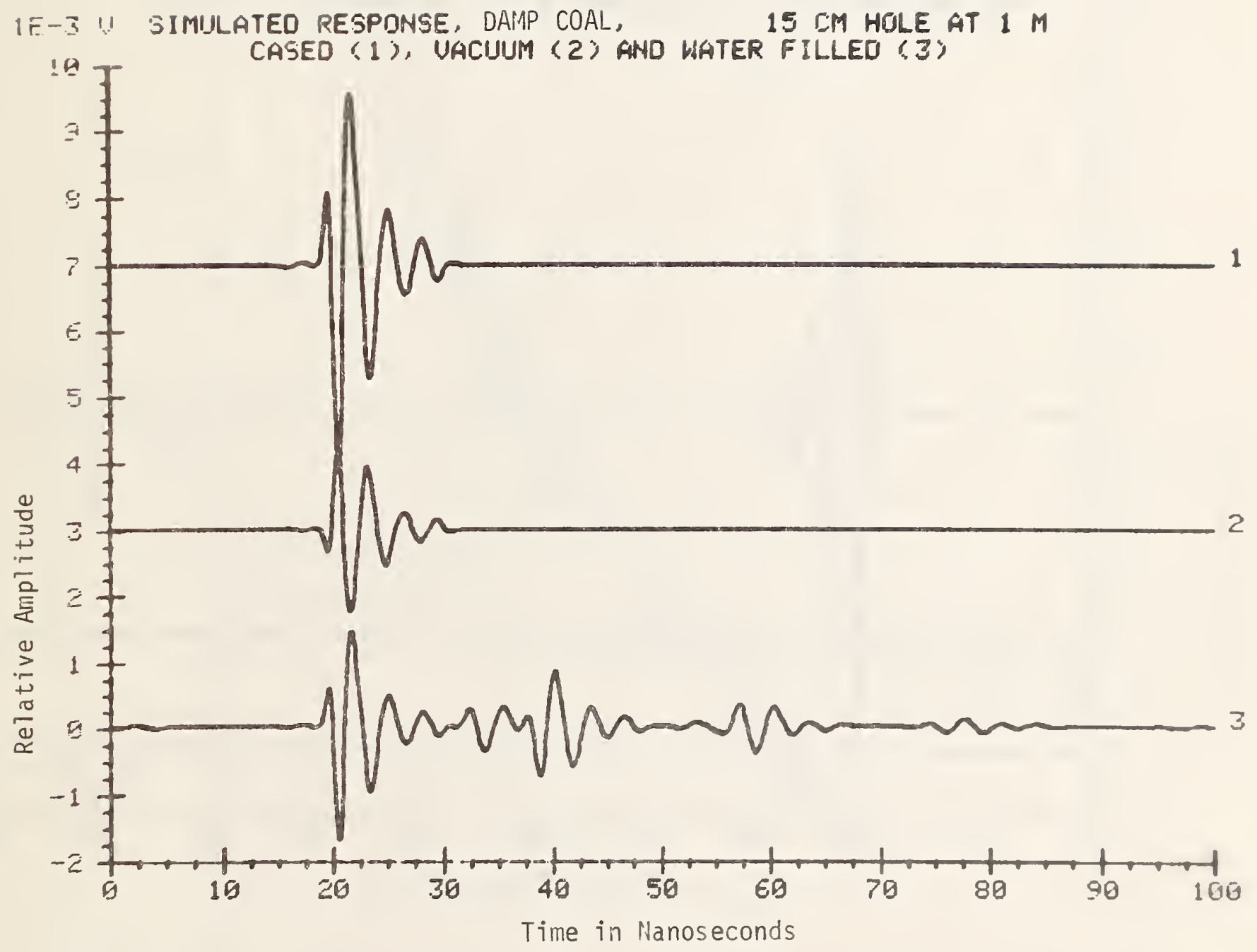

Figure 27. Simulated response in relatively damp coal for a $15 \mathrm{~cm}$ radius hole at 1 meter range. (1) cased hole, (2) air-filled hole, water-filled hole. 


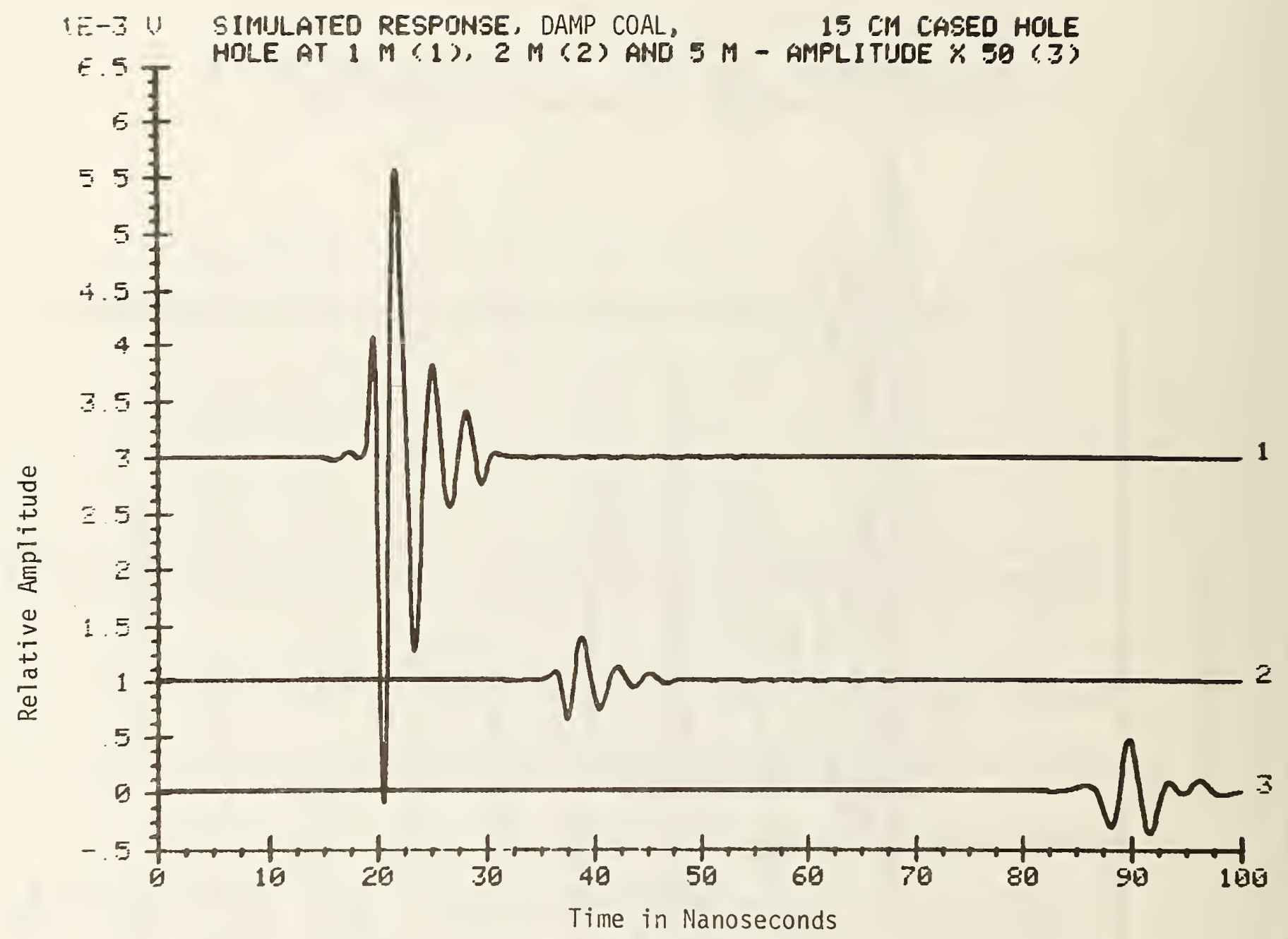

Figure 28. Simulated response in relatively damp coal for a $15 \mathrm{~cm}$ radius hole at a range of 1 meter (1), 2 meters (2), and 5 meters (3). The amplitude of the response at 5 meters has been amplified 50 times. 


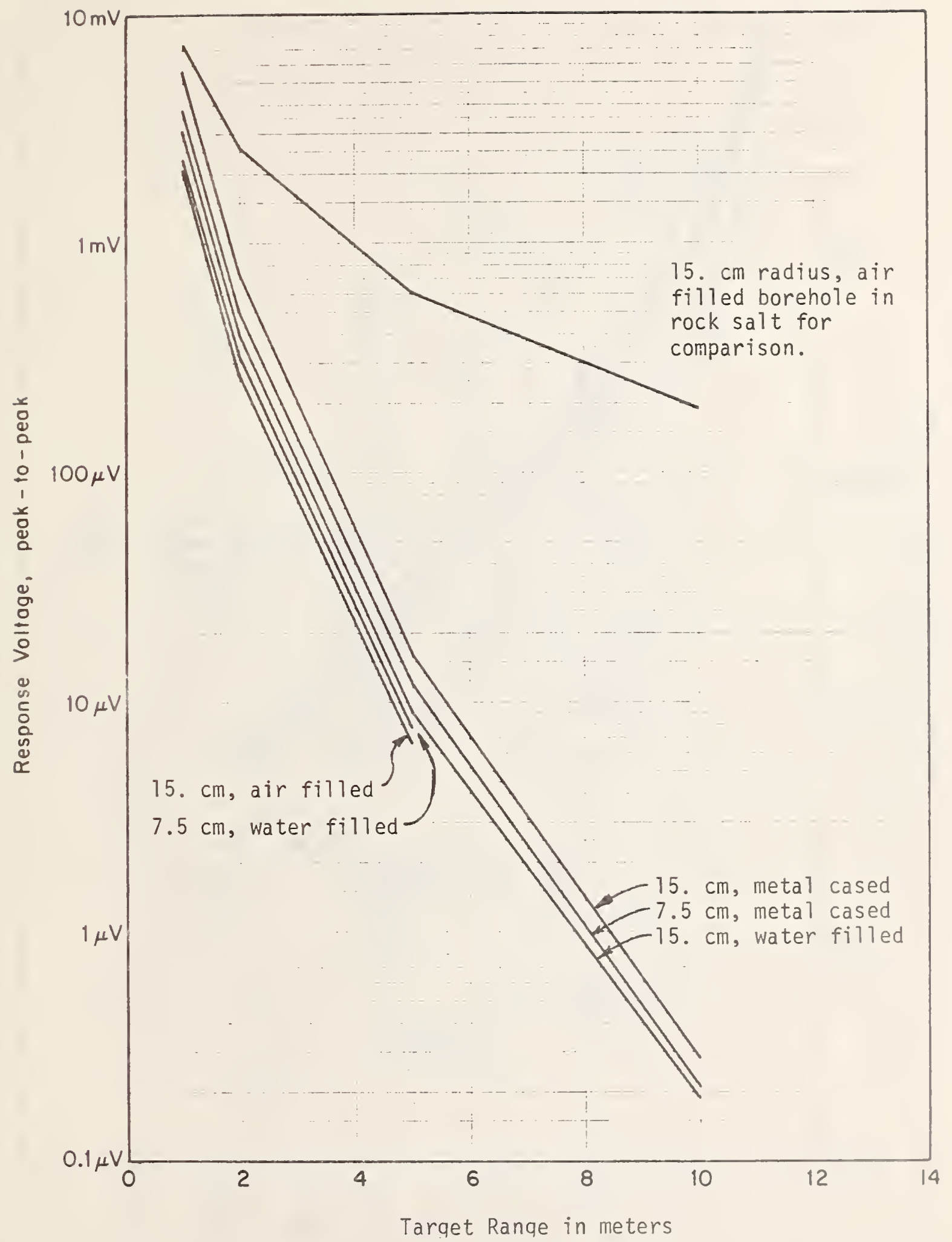

Figure 29. Simulated radar response in relatively damp coal for a 1.0 volt, 1 nanosecond input pulse reflected from a borehole. The given dimension is the borehole radius. 


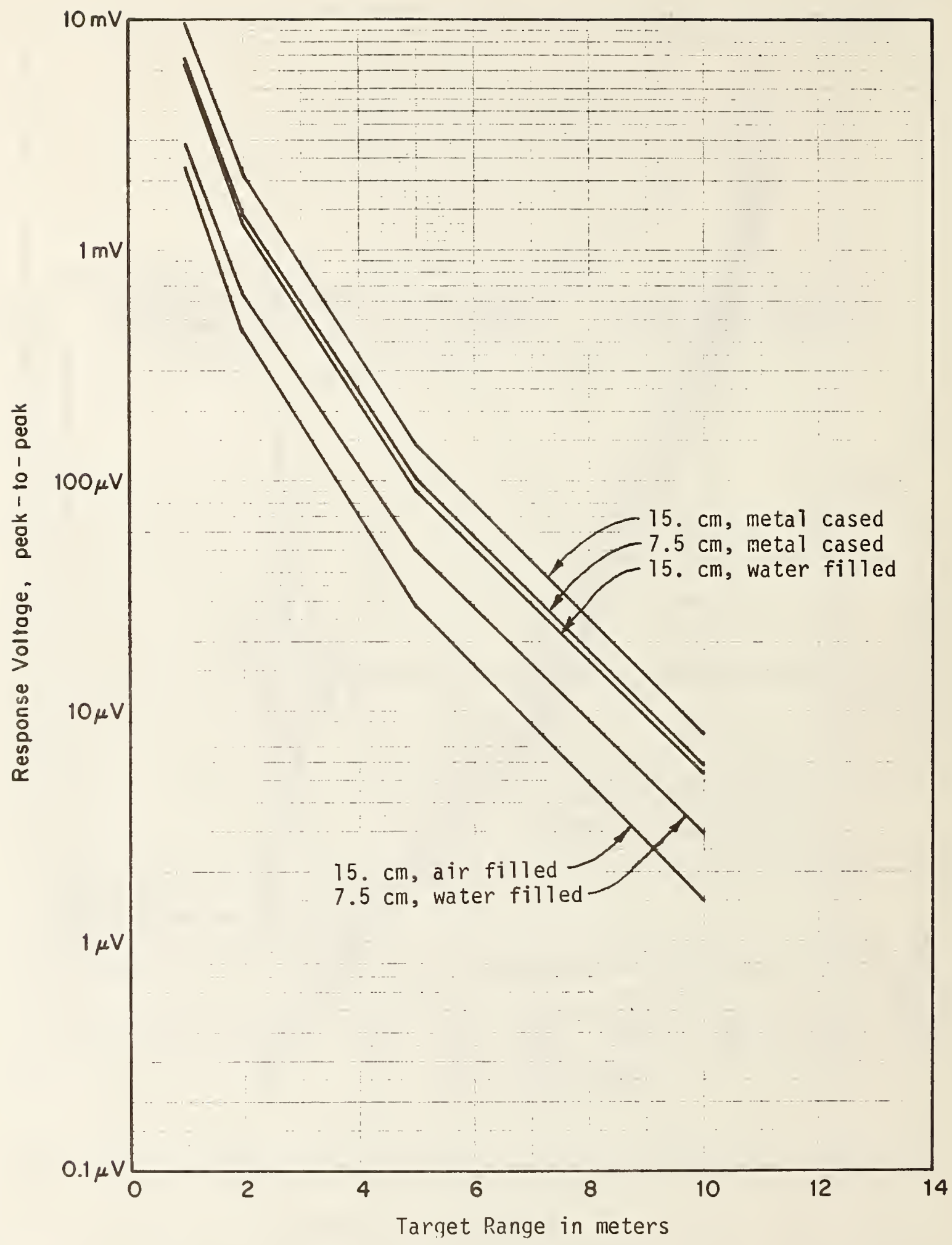

Figure 30. Simulated radar response in relatively dry coal for a 1.0 volt, 1 nanosecond input pulse reflected from a borehole. The given dimension is the borehole radius. 


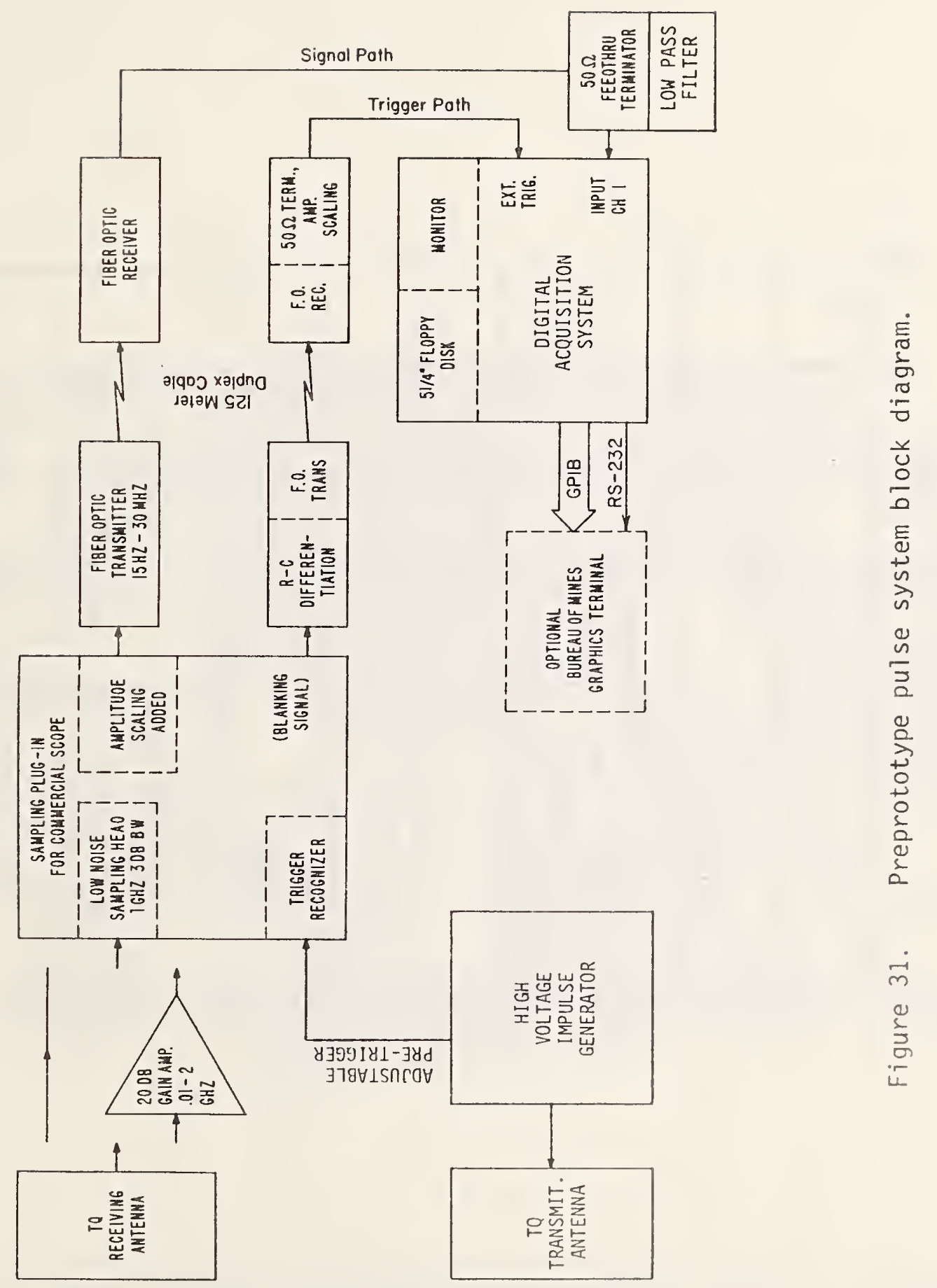




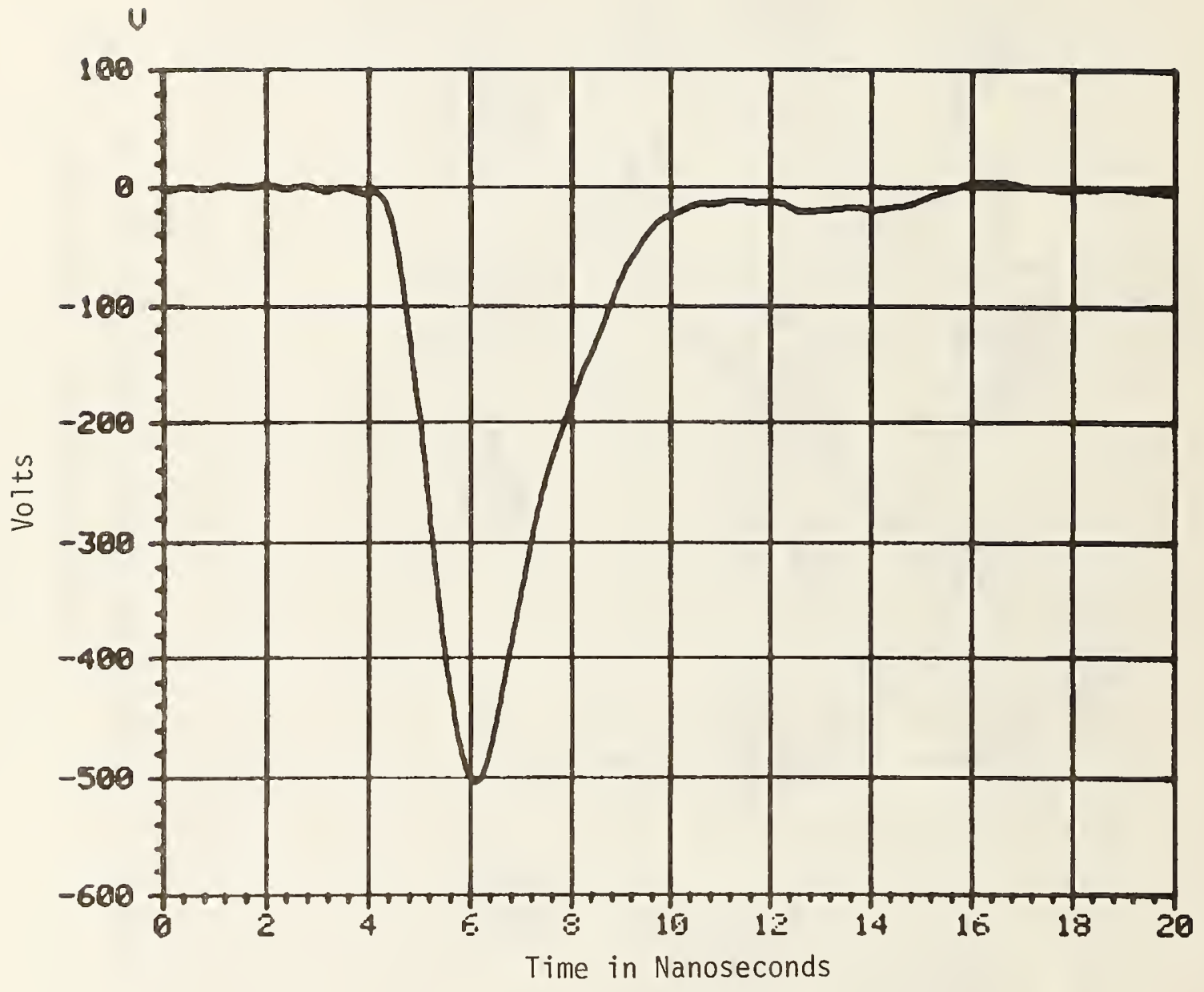

Figure 32. Output waveform of the high-voltage impulse generator. 


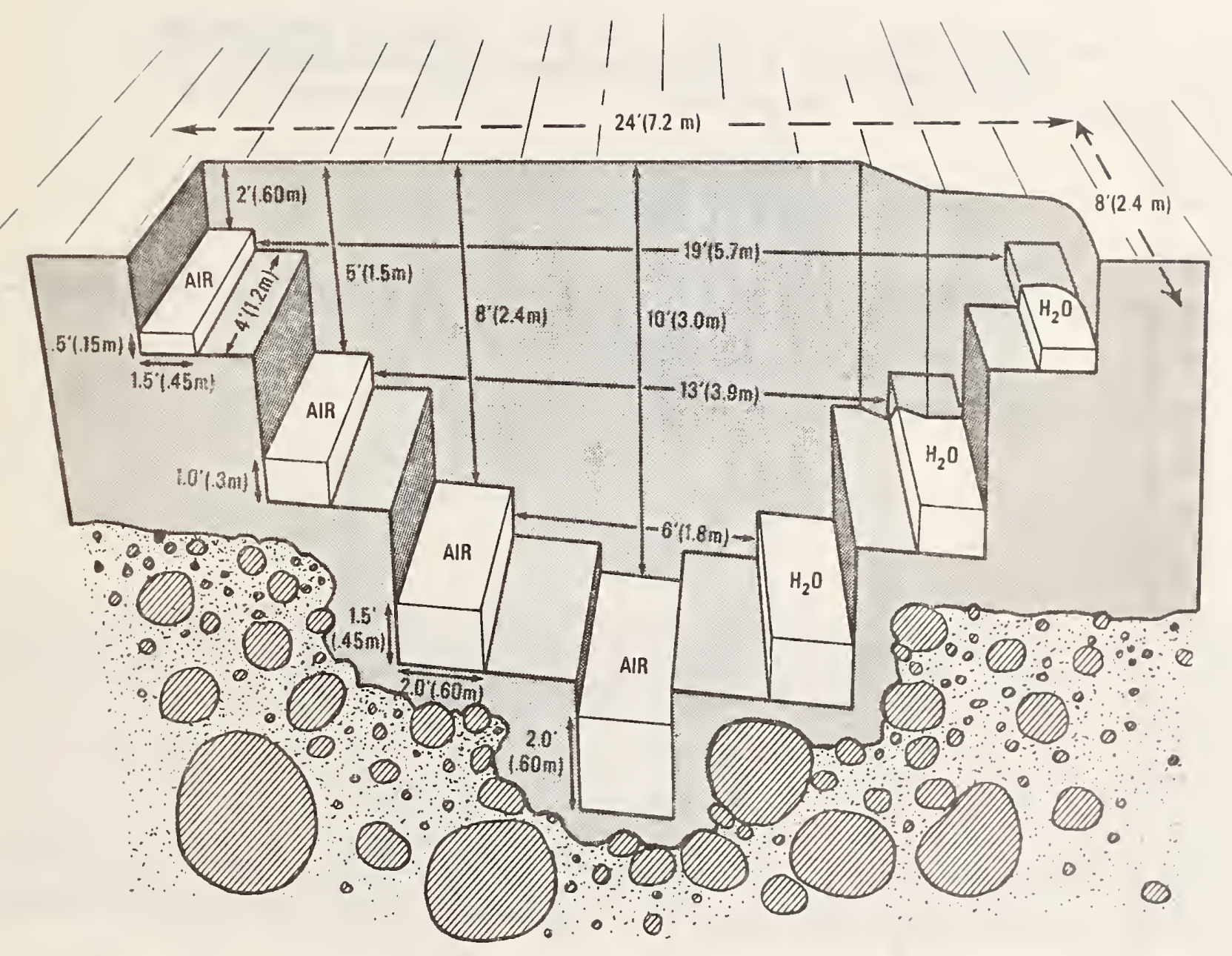

Figure 33. NBS range for subsurface anomaly measurements. 


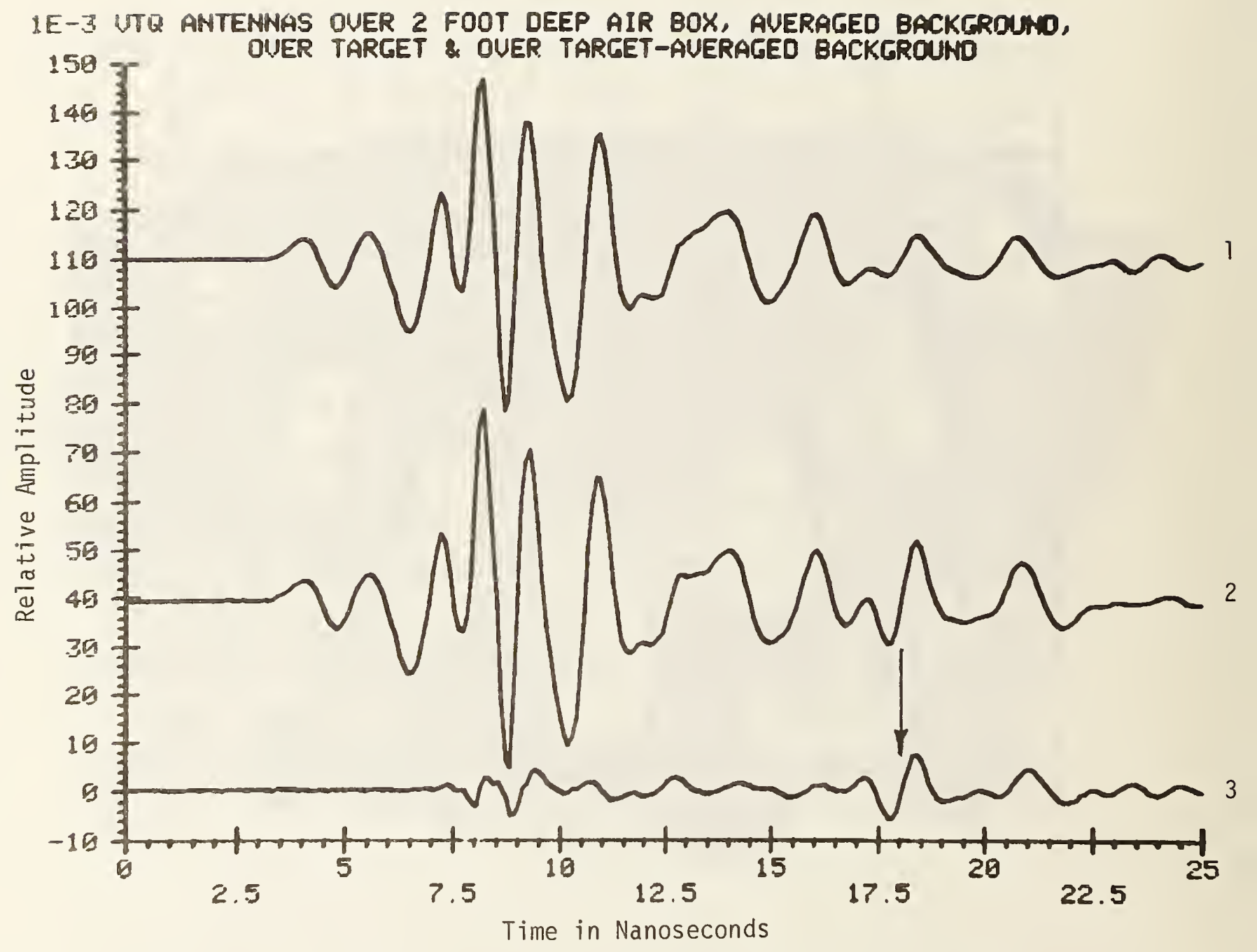

Figure 34. Pulse system response averaged over a sequence of 26 measurements (1). Single response from over the 0.6 meter deep air-filled target (2). The resulting response showing the target signal enhancement (arrow) obtained by subtracting the sequence average from the response at one position (3). 


\section{TQ ANTENNAS WITHOUT PRE-AMP SCANNING OUER 2 ' TARGET \\ FILE TQ65MN.5 FROM 8 TO 2.49023E-98 MINUS TQ6SMN.5AU}

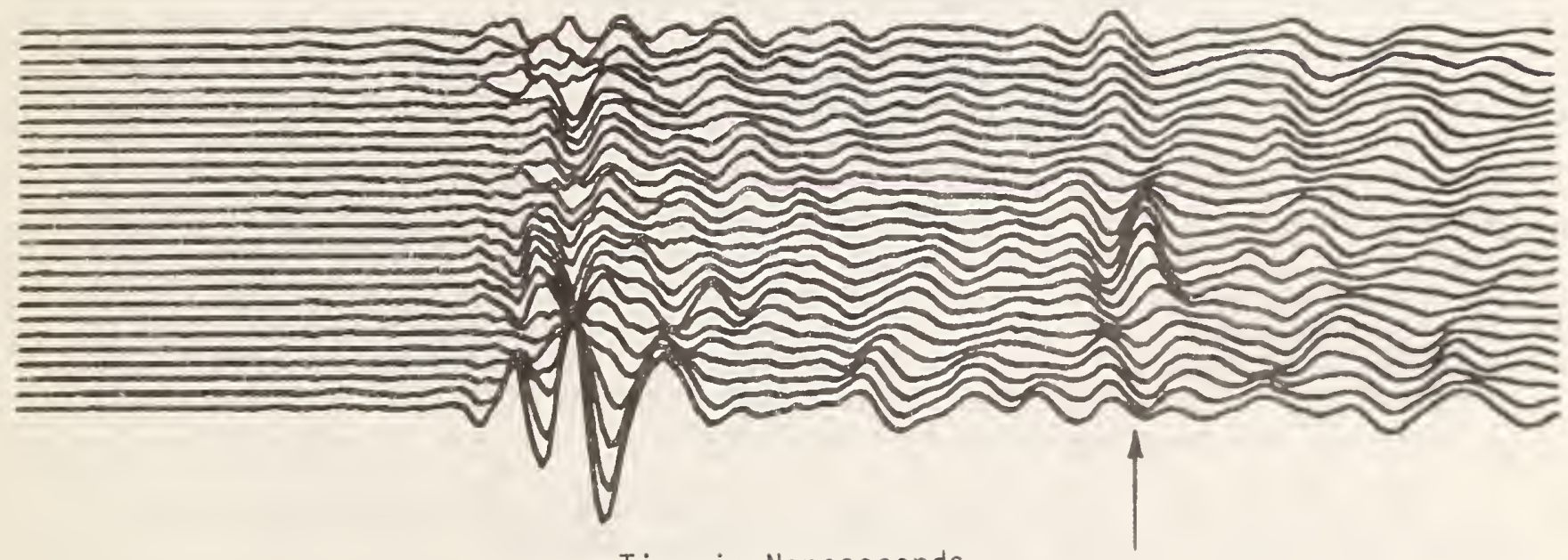

Time in Nanoseconds

Figure 35. A stacked series of modified responses obtained by subtracting the average response of the series from each measured response. Target signal at arrow. 


\section{APPENDIX A}

\section{COMPUTER PROGRAM FOR SIGNAL-TO-NOISE RATIO, AS A FUNCTION OF DISTANCE (RANGE) TO A SUBSURFACE TARGET}

The program listing, written in BASIC language, is included in this appendix. Some results from the program are shown in figure 14, which shows curves for the signal-to-noise ratio, $S / N$, as a function of distance for specific coal "atmospheres," specific targets, and a specific radar system model.

The radar system has some constant parameters and others as input variable. The constants are given in statement 20 (S20). The variable parameters required are frequency (S60), antenna dimensions (S90), dielectric permittivity and loss tangent of coal (S120), coal conductivity (if the dielectric tangent was entered as zero), and one of the five targets considered as described in S240 and S260, and the target dimension S550. The source power, $10 \mathrm{mh}$, and the frequency, $500 \mathrm{MHz}$, are assigned in $\$ 116$ and $\mathrm{S70}$, respectively.

The noise is that for a classical CW radar with Johnson noise in a $1 \mathrm{MHz}$ bandwidth augmented by an $8 \mathrm{~dB}$ noise figure, i.e., multiplied by 6.31. The noise power is calculated as D6 in $\$ 490$.

The received power is calculated as $S 4$ in $S 670$. The $S / N$ is calculated in line $\$ 680$.

$S / N$ for other radar systems may require running the program. However, for simple cases, the graph may be reinterpreted. For example, if the source power is increased by $10 \mathrm{~dB}$, the $\mathrm{S} / \mathrm{N}$ at any range is increased by $10 \mathrm{~dB}$. If the noise is $5 \mathrm{~dB}$ greater, then $S / N$ is $5 \mathrm{~dB}$ smaller.

With different target dimensions and a different frequency, it is necessary to rerun the program. The statements from 750 to 1800 are used to calculate target cross section as a function of frequency and dimensions. The methods are approximate. The strip validity is discussed in the text. The method for the cylinder cross section branches to one of three methods depending on the ka. The small and large cases are well known approximations. The intermediate case, $\$ 840$ to $\$ 1020$, uses forward recursion to evaluate the cylinder functions (see S1350, S1360), which is ultimately an unstable procedure for $S / \mathbb{N}$. However, for the present usage in the intermediate range, 0.05 $<k a<5.99$, the resulting truncated Bessel function series is accurate within $\pm 1 \%$ except for an anomaly for ka near unity.

It may be noted that the ratio of source power to noise power, with no propagation loss, is $0.01 \mathrm{~W} / 0.0252 \mathrm{pW}$, or about $116 \mathrm{~dB}$.

The ultimate range of a radar is often taken to be $S / N=0 \mathrm{~dB}$. With signal processing, it may be at less than $0 \mathrm{~dB}$. With clutter, it may be considerably above $0 \mathrm{~dB}$. 


\section{Example of Results of the Radar Range Program}

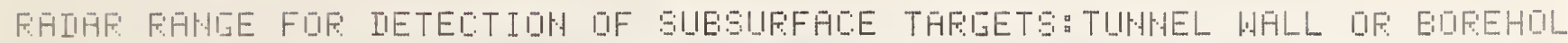

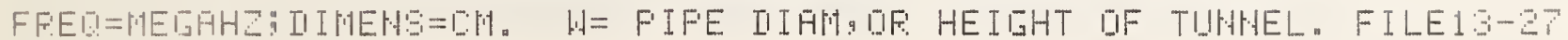

IHFIIIT FE

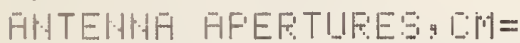

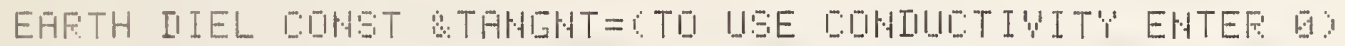
$\exists$
E. 1
3.9197932
回 1491315

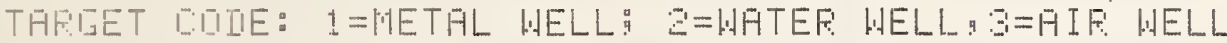

LIIE= 1 4=WHLL WITH WHTEF $5=$ WHLL WHIF HIISE FIILEE IIE G

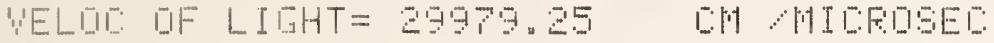

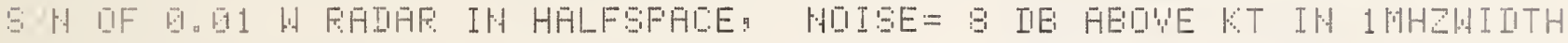

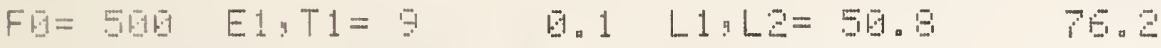

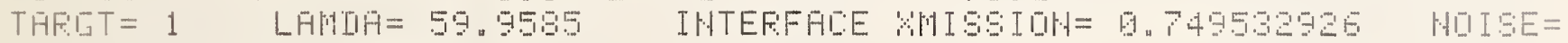
2. $230 \mathrm{EE}-14 \quad \mathrm{Q}=1$ $\omega=$

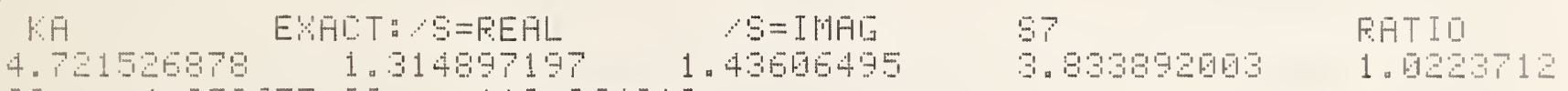

26 4.

$46 \quad 1.54080-94 \quad 97.97952724$

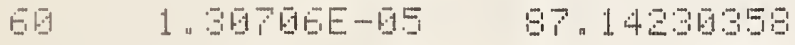

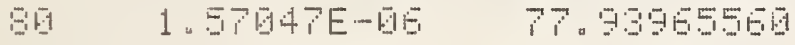

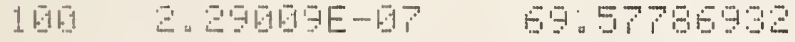

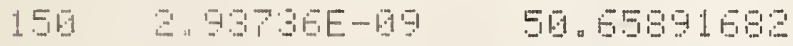

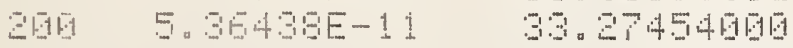

256 1.196E-12 15.7.19459

$301929795 E-14$ 国 71997279

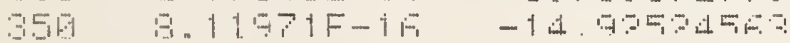

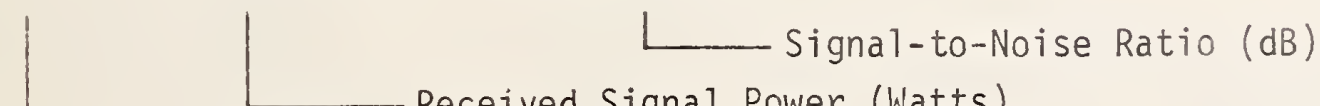

Received Signal Power (Watts)

Range $(\mathrm{cm})$ 
5 F:EN F:AIIAF: F:AHIIE

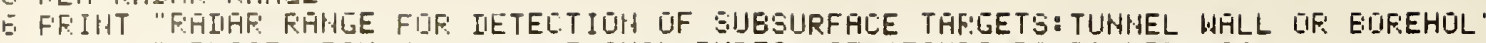
$\vec{r}$ FEW H.E. EUSSEH, IIV 723.62 , HATIOHAL BUREAU OF STANIARDS,BOULDER CG.

16 FEN FILE $10-27$ FS MODIFIED $12,12 \% 80$

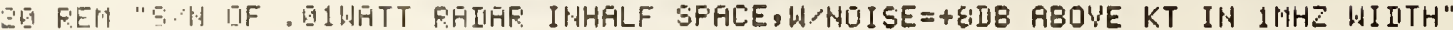

$361=2979.25$

46 FFINT "FFEQ=MEGAHZ; DIMENS=CM. W= PIPE JIHM, OR HFLF HT OF TUNHEL.FILEI0-27"

SIG FEN TI CHAHGE UHITS, SHY', METERS REPLACE VI BY W1 IN METERS/MICROSEC

E. FF:IHT "IHFUT FG";

TD IHFUT FO

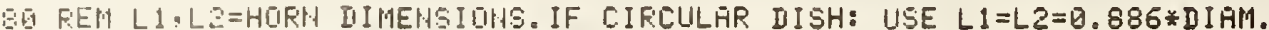

GG FF:IHT "AHTEHNA RFERTUFES, LH=";

1 1GT IHFUT LI, LZ

11 GEM FE=EFFECTIVE CW FERAR POWER IN WATT

$116 F \cdot B=0.01$

12 GFINT "EHRTH DIEL COHST \&THNIIT=(TO USE COHDUCTIVITY ENTER O)";

13 INPUT EI:TI

140 IF T1 10 THEN 190

15IV FFIHT "IHFUT EDNDUE. MHUS METER"

10 IHFIJT 51

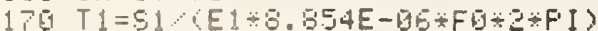

1EG FEM LOMFLY REFRALTIVITY NS\&NE

$1911=50 F(5,5 \div E 1)$

$260 \quad T=50 F(1+T 1 \div T 1)$

$216+15=11 \div G 0(1+T)$

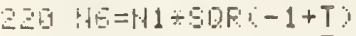

GE FEIHT E1:T1:N5:NE

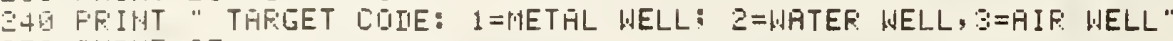

GE IHFILT E:?

2E FFIHT "LQDE="C7" 4=WHLL WITH WHTER; $5=$ WHLL WAIR ";

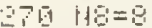

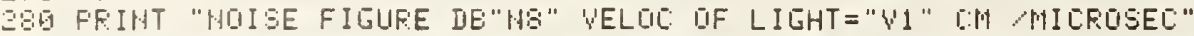

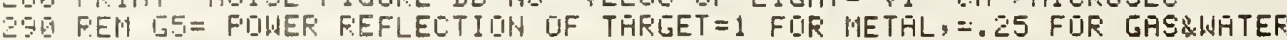

$36,5,5=1$

31 IF 0.72 THEN 336

311 IF $\quad[7=2$ UP $L P=4$ THEN 315

$13 \quad 5=(4.5-1)(1,5+1))+2$

31350150

$15 \quad 5=(15-9)(15+9)+2$

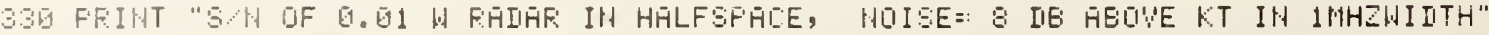

$5 L=1, F$

$3 E$ G $1=2 \div F^{\prime} I L$

$3706=k 8+1.5$

HEIG FEH HT $=$ TT HOISE IH 1 MHZ WIITH

3. $1.7=4 E-15$

4EG FEM 1-WH' FOUR YMISS. DIF IHTEF:FHI:E = I

$410 I=4 \div 1.5(1+1.5) \div(1+15)$

$42 G$ FEM II SFEFEIOH MULTIFLIEF:

$4.10 \mathrm{i}=0.5$

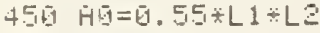

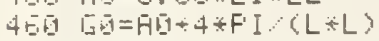

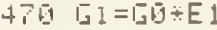

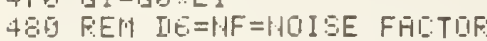

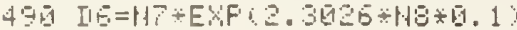

5GG FFIHT "FG="FG"E1,T1="E1:T1"L1,LZ="L1:LZ

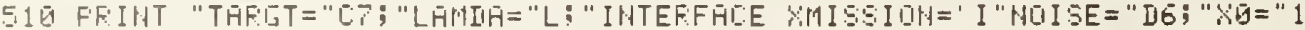

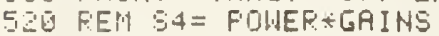

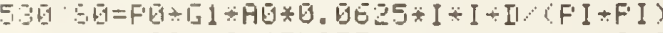

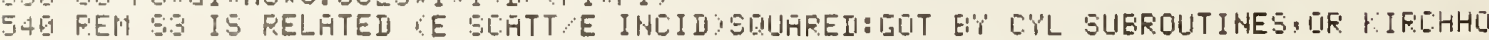


550 H. IIIT "W=";

다 1 I IFUT W

$5, \bar{U}$ IF $2: 4$ THEN 770

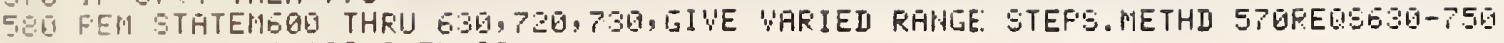

50 FII R $=20$ TO 100 STEF 20

E.บ1 GUTO E30

$615 \quad F=F+50$

E. IF $R=1565$ THEN 740

E.36 GOSUE L.7 OF 750,750,750,18.30, 16.30

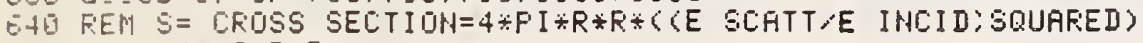

$5.50 \quad 3=53 \div 4 * F I * R * R$

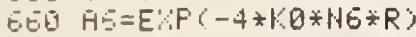

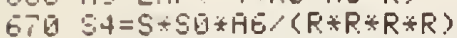

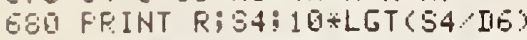

E. IF $R: 101$ THEN 710

PGण IF $R \geqslant 100$ THEN 610

P1G IIEXT R

$70 \mathrm{~F}=100$

730 TITO E.10

74 EH EH

TE $63=5 \mathrm{~F} F$

TEG FETUFH

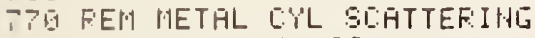

SBL III J[ 15], Ý[16]

FII IHT " KA

ह1. $\overline{\mathrm{H}}=W+\overline{\mathrm{C}} .5$

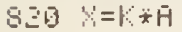

BU IF $X<0.05$ THEH 920

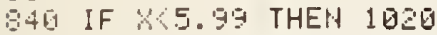

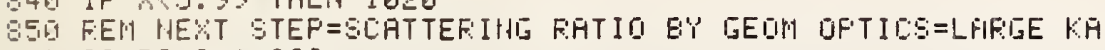

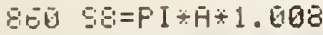

87日 F $B=5$ (PI $\div$ 月)

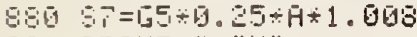

896 FFINT " "X"

900507000

910 FEM ENHLL EUL SLHTTEFCFA'ILEIJH)

92 II $1=$ LDI

1.15 II $=[1]+10.5772157$

$945 \quad I I=I 2 \div I 2+6.25 \div F I \div F I$

$5501=-1.25 \% F I * F I D$

$910=5.5 \% \mathrm{FI} \div \mathrm{I} 2 \mathrm{II}$

$971 \quad F B=51+51+52 * 52$

98 G $S=G+F B(F I \div K)$

G $F=4 F F(F I \div Q)$

16日6 FFIHT $2 ; 51 ; 52 ; 57: F 7$

1610 G0T 5 G日

1.Z FEMEESEL (INTEFMEIIHTE LUL)

$105 \mathrm{x}=\mathrm{x}$

164 IF $X>2.99$ THEH 1150

$1650 \quad y_{1}=8$

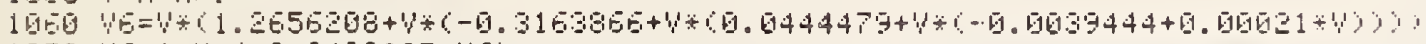

$1610=1+1+2.249999+46$

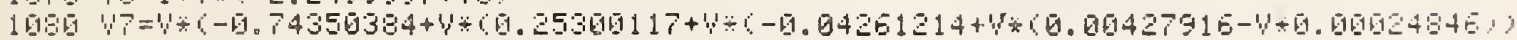

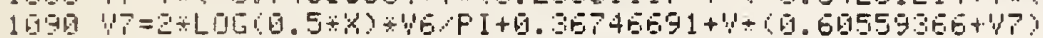

1160 W

$1116 \quad y=x \div 6.5+4 *(-0.56245985+4 \div 40)$

$11269=4 \div 2.1682799+4 *(-1.3164827+4 * 6.3123951+4 \div(-6.0400976+4 * 0.6027873)$

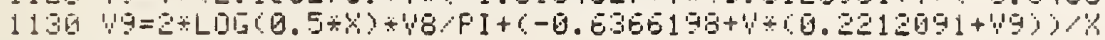

1140 10TO 1290

$1150 y=3 \%$

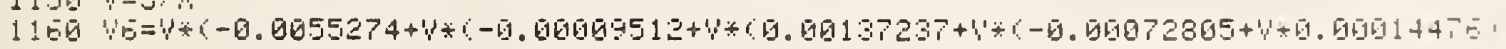


1176 W $=0.79785456+V *(-7.7 E-67+46)$

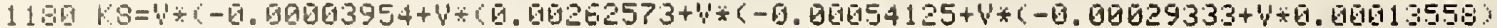

$1190+5=x-7.7539816+v \div(-0.04166397+k 8)$

$126198=518 x$

$121047=5 I N K 8 * V 6 \times 2=$

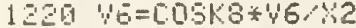

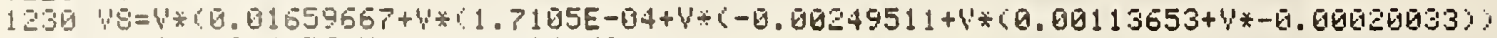
$1240 \quad \forall B=0.7878345+4 *(1.5 E E-B E+48)$

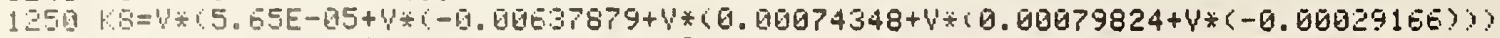

$12618=8-2.35619449+V *(6.12499612+K 8)$

$1270 \quad y=5 I N(K 8) \div 48 / X 2$

$1286 \quad 4 B=105(K 8) * V 8 / x 2$

$129 \mathrm{FEM}$

$136 \overline{6}, \pm[1]=V 6$

$1316]+2]=18$

130 i[ 1$]=42$

130 II $[2]=1 / 9$

134 GOF $F=2$ TO 15

$1350 . J[P+1]=(2 \div P-2) * J\left[F^{\prime}\right] / X-J[F-1]$

$13 F[F+1]=(2 \div P-2\} \div[F] Y-Y[F-1]$

1300 IF I[F] $-9 E+65$ THEN 1400

$13 G$ HEYT $F$

14 回国 $F^{\prime} 1=F^{\circ}$

$141051=0$

$14258=0$

$1430 \quad 1=-1$

1440 FIF $F=1$ TE $F^{\prime}$

$1450 \mathrm{~N} N \mathrm{~N} \div-1$

$14 E[\quad[Z=\mid[F] \div J[P]$

147 III $02+Y[F] \div \mathrm{Y}^{\prime}[\mathrm{F}]$

140 IF F I THEN 1510

$1496 \quad 57=1$

156 5150 1526

$151007=2$

$15-6 F_{1}=57$

$15301=51-F_{1}+125+1$

$15490=51 ;$ If $\left.F^{\prime}\right] \div\left[F^{\prime}\right] * 14$

1550 GEN IF IF] $[F-1 E+0.5$ THEH 1575

1570 HEWT $F$

$15 B \quad F B=51+51+89+53$

$15905=5.5 B$ (PI +1$)$

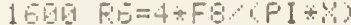

1E10 FEINT Y:S1;S2:57FE

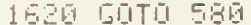

1E. FEN IFLHHOFF -FFESHEL FIELI OF A STFIF, THFE \#II,FILE 30

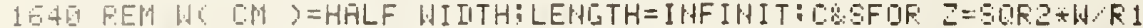

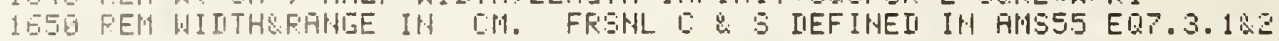

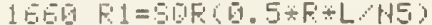

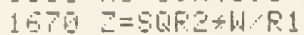

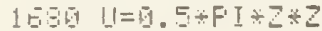

$1696 \quad F=1+5.925 * 212+1.792+2+3.104+2+2)$

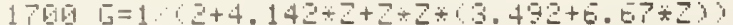

$171651=5 I H(U)$

$17251=[015(U)$

$1730=0.5+F \div 51-5 \div \mathrm{C} 1$

$17+5=0.5-F+C 1-[* 51$

$17 E-6=0.5 *(C * L+59 * 59)$

$177003=55 \div 2$

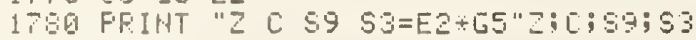

1790 RETURN

$18 \bar{G}$ EHD 


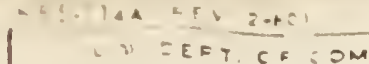

BIBLIOÉFAPHIC DATA

SHEET ISEe ins? UEcLions

4. TITLE ano SUETITLE

Microwave Detection of Lost Wells and Unknown Water-Filled Voids in Coal Mines

S. AUTHOR(S)

D.R. Belsher; R.H. Mclaughtin; A.G. Repjar; and H.E. Bussey

6. PERFORMING ORGANIZATION (If joint or other thon NBS, see instructions)

NATIONAL BUREAU OF STANDARDS

DEPARTMENT OF COMMERCE

WASHINGTON, D.C. 20234

9. SPONSORING ORGANIZATION NAME AND COMPLETE ADDRESS (Street, City, Stote, ZIP)
7. ContracvGrant No.

8. Type of Repori \& Period Covered

10. SUPPLEMENTARY NOTES

Documeni describes a computer program: SF-185. FIPS Software Summary, is attached.

11. ABSTRACT (A 200-word or less foctuol summory of most significont informotion. If document includes o significon: bibliogrophy op literoture survey, mention it here)

Work on contract $\mathrm{H0272007}$ is summarized for the period of January 1979 through March 1984. The development of improved antennas useable with both a puise system or an FM-CW system is described. The development of a field prototype puise sampling system is described. Initial theoretical work on the problem of dielectric loading of antennas as well as a study of potential system range is included.

12. KEY WORDS (Six io iwelve entries; olphodeticol order; copitolize only proper nomes: ond seporote key words oy senicolons, antennas; dielectric load; puised system; radar range

13. AVAILABILITY

Unlimited

For Official Distribution. Do No: Release to NTIS

Order From Superintendent of Documents, U.S. Government Printing Office, Washıngton. D.C. 20402.

Order From National Technical Information Service (NTIS). Springfielc VA. 22161
14. NO. OF

PRIPTEO PAGES

86

15. Price 


$$
\Xi=
$$

